\title{
An integrated model for fire sales and default contagion
}

\author{
Nils Detering ${ }^{1} \cdot$ Thilo Meyer-Brandis $^{2}$ (D) $\cdot$ Konstantinos Panagiotou ${ }^{2} \cdot$ Daniel Ritter $^{2}$
}

Received: 5 April 2019 / Accepted: 12 June 2020 / Published online: 27 June 2020

(c) The Author(s) 2020

\begin{abstract}
Fire sales and default contagion are two of the main drivers of systemic risk in financial networks. While default contagion spreads via direct balance sheet exposures between institutions, fire sales describe iterated distressed selling of assets and their associated decline in price which impacts all institutions invested in these assets. That is, institutions are indirectly linked if they have overlapping asset portfolios. In this paper, we develop a model that helps us understand the joint effect of the two contagion channels and investigate structures of financial systems that promote or hinder the spread of an initial local shock. We first consider the contagion process for an explicitly given system and then derive our main results for random ensembles of systems whose macroscopic statistical characteristics of defining parameters are close to each other. In particular, we model direct exposures by means of a random graph. Our approach ensures robustness to local uncertainties and changes in the system. We characterize resilient and non-resilient system structures by criteria that can be used by regulators to assess system stability. Moreover, we provide explicit capital requirements that secure the financial system against the joint impact of fire sales and default contagion.
\end{abstract}

Keywords Systemic risk · Financial contagion · Asset fire sales · Price-mediated contagion · Default contagion $\cdot$ Capital requirements

JEL Classification C02 · E61 · G01

Thilo Meyer-Brandis meyerbra@math.lmu.de

Nils Detering

detering@pstat.ucsb.edu

Konstantinos Panagiotou

kpanagio@math.lmu.de

Daniel Ritter

ritter@math.Imu.de

1 Department of Statistics and Applied Probability, University of California, Santa Barbara, CA 93106, USA

2 Department of Mathematics, University of Munich, Theresienstraße 39, 80333 Munich, Germany 


\section{Introduction}

Today's financial networks are characterized by various types of dependencies between financial institutions and a complex network structure. While these connections go along with attractive business opportunities for each single institution, they also bear the risk of spreading and amplifying local shocks through large parts of the system, which is known as systemic risk. A particular challenge is to study the interplay and mutual reinforcement of various types of contagious connections. Two of the main drivers of systemic risk that we focus on in this paper are the contagion channels default contagion and fire sales (see [28] for instance).

Default contagion describes insolvency transmission between direct neighbors in a financial network due to the inability to meet financial obligations. That is, if a certain bank has to declare bankruptcy and hence cannot (fully) repay loans or other pending liabilities, then all exposed institutions in the system have to write off their losses and can get into financial distress themselves. Although default contagion takes place between direct neighbours in the network, it was one of the main insights from the financial crisis that also second order effects play an important role when assessing an institution's stability with respect to certain shock events. This is the main difference of systemic risk as compared to classical counterparty risk: not only an institution's contractual partners are important but also their business connections and so on, which the first institution has no influence on or even knowledge of.

The contagion channel fire sales describes a similar effect: it can happen if for some reason one or more investors sell off a large number of shares of a certain illiquid asset which causes a decrease in the asset's share-price. Hence, by mark-to-market of portfolio values, this leads to losses for all institutions invested in the asset and thus possibly causes further asset sales in order to comply with external regulations or internal strategies. This on the other hand drives down prices even further and the cycle of fire sales goes on and on. In contrast to default contagion, fire sales can transmit distress from one institution to another even without a direct business relationship. Each institution that holds an asset portfolio of some kind is then exposed to all other institutions with a (partially) overlapping portfolio. However, due to second order effects, institutions can even be exposed to other institutions without having any overlapping asset holdings. In contrast to default contagion, fire sales can start while all institutions in the network are still solvent but some of them choose to sell assets either voluntarily or because of regulatory requirements.

Clearly, the two described effects can further amplify each other. If, for instance, during a fire sales cascade an institution goes bankrupt, as described above its creditors have to write off their loss and may be driven into selling parts of their asset portfolio thus sparking further fire sales. The underlying motivation for this paper is thus to model the interplay of default contagion and fire sales and particularly to understand which structures of direct exposures and overlapping asset portfolios promote or prevent the spread of local stress in a system.

\subsection{Related work}

One of the first papers to rigorously address contagion effects in financial networks was the seminal work by Eisenberg and Noe [20]. In its original form their model (the ENmodel) describes the channel of default contagion without bankruptcy costs and under a perfect claim enforcement technology. The main insight was the existence (and sometimes uniqueness) of a payment vector that clears liabilities in an explicitly given interbank network. The popularity of the EN-model is partly due to its tractability which allows for a number of 
extensions including for example default costs, fire sales and crossholdings between banks (see $[12,24,32,34])$.

Other lines of research addressed the question which network structures promote a cascade of defaults. For example the authors in [1,2] compare stylized network structures, the ring and complete network. They show that complete networks can absorb small shocks better but large shocks are amplified. The authors of [9] present a more general framework that allows to compare a larger set of network structures, including the empirically relevant coreperiphery network. In addition, in contrast to previous studies they account for the presence of bankruptcy cost. Their main insight is that for highly capitalized systems a more diversified network structure is better, while for poorly capitalized systems diversification can be harmful and create potential for larger amplification of bankruptcy cost.

Another line of research, which started in [23] and continued for example in [16,17,19], uses asymptotic random graph techniques. Similarly to [1,2,9], the objective is to gain understanding of which network structures promote systemic risk and which capital requirements are suitable to prevent large cascades. Rather than considering a concrete network configuration, these works derive results for a whole family of random graph configurations whose macroscopic statistical characteristics are calibrated to resemble an originally observed structure. That is, statements about the final state of the system are possible in terms of global statistical quantities only, and independent of the precise structure on a microscopic level; this makes this approach robust to local uncertainties as well as changes over time and thus attractive from a regulatory perspective. Moreover, for large systems a natural notion of resilience emerges that is not based on any arbitrarily chosen parameters, but considers an arbitrarily small initial shock and its effect on the global system.

In terms of fire sales [25] consider an economy consisting of multiple banks and multiple assets with different liquidity levels to study first order effects of fire sales (the asset sales of one bank creates a balance sheet hit to another bank only if it holds the same asset). In [10] the authors introduce a systemicness matrix to quantify the aggregated effects of liquidation when banks hold overlapping portfolios. Their analysis includes the impact of second order effects. Using a large system approach [18] proposes a model for fire sales that also accounts for higher order effects. The authors derive asymptotic results about the fraction of finally defaulted institutions, the number of sold asset shares and the ensuing final price impact. Also see [8] for a similar albeit much simpler framework for investigating fire sales on a random network by a branching process approximation.

While the asymptotic methods line of research accomplishes to characterize favorable and unfavorable system structures, it has so far only been possible to model one contagion channel at a time. It is the aim of this paper to present the first integrated model for default contagion and fire sales that uses the asymptotic approach to provide insight into the effect of certain structural characteristics on system stability. In an Eisenberg-Noe setting this integration has been done in [12] and in [34]. In [5] the authors endogenize possible intervention to stop contagion that propagates through fire sales and default losses.

\subsection{Contribution}

In this paper we study the joint effects of default contagion and fire sales and their impact on system stability as well as systemic risk management.

Integrated model for default contagion and fire sales Our main objective in this paper is to specify and study a stochastic network model for default contagion and fire sales. To prepare the ground for such a model, we first state a deterministic model for simultaneous 
default contagion and fire sales such that in particular the two can amplify each other. At this we assume the following underlying parameters for each institution $i \in[n]=\{1, \ldots, n\}$ : Its initial capital $c_{i}$, which is reduced by some individual exogenous shock $\ell_{i}$, a list of direct exposures $\left(e_{j, i}\right)$ to other institutions $j$ such that $i$ 's capital is reduced by $e_{j, i}$ if $j$ defaults, and a vector $\boldsymbol{x}_{i}=\left(x_{i}^{A}\right)$ of numbers of shares held of each asset $A$. In addition every institution is assigned sale functions $\rho_{i}^{A}$ that abstractly describe asset sales of each asset A due to regulation or other constraints as institution $i$ incurs losses. The sale functions are allowed to vary across different institutions, as for example hedge funds, investment banks or commercial banks might have a very different asset sales behaviour, and asset classes, for example in order to distinguish between sales of assets with different liquidity levels. Lastly, a function $h^{A}$ is given that specifies the impact of sales on asset A's price-assuming that exogenous price changes are negligible during the contagion process and $h^{A}$ is the sole driver of the asset price. We only require minimal assumptions on $\rho_{i}^{A}$ (non-decreasing) and $h^{A}$ (continuous and non-decreasing) to derive our first result about the set of finally defaulted institutions and the vector of finally sold shares in terms of a fixed point equation. In addition we assign to each institution $i \in[n]$ a value $s_{i}$ of systemic importance. This value does not influence the contagion process but allows for a more refined risk analysis based on the set of finally defaulted institutions after a cascade.

The stochastic model The previously mentioned results allow us to compute the final damage to any explicitly given financial system. The actual focus of this paper, however, is to go one step further and to model a whole ensemble of systems simultaneously to understand which structural properties in a system expedite or inhibit the spread of distress. More precisely, we aim at performing an asymptotic analysis for a financial system that inherits the model features and flexibility of our deterministic model. Our model for the financial system exhibits a degree of flexibility and heterogeneity to capture observed features that was previously unseen within the literature on asymptotic random graph models for systemic risk. We achieve this by the following means:

1. We randomly rewire direct links in the network according to the following method: We assign to each institution $i$ a type $\alpha_{i}$ out of finitely many types $[T]:=\{1, \ldots, T\}$. Institutions of the same type share a certain similarity, for example they could be residing in the same geographical region, be of a similar institutional type or in the same layer of a tired network such as core-periphery. Classification of certain institutions as systemically important financial institution (SIFI) is also possible. In addition we assign in-weights $w_{i}^{-, \alpha}$ and out-weights $w_{i}^{+, \alpha}, \alpha \in[T]$ and draw a random link from $i$ to another institution $j$ of type $\alpha_{j}$ with a probability proportional to $w_{i}^{+, \alpha_{j}}$ and $w_{j}^{-, \alpha_{i}}$. That is, $w_{i}^{ \pm, \alpha}$ describe the tendency of $i$ to send/receive links from institutions with certain types. They can be calibrated to the observed in-/out-degree in a concrete network configuration. We refer to Sect. 6 for details on the estimation of the parameters. This set of parameters ensures that important statistical properties of this random graph are in line with observed real financial networks with a complex tired structure especially on a global level. While in some countries a power law or core/periphery degree sequence was observed (see [14]), one degree sequence is not enough to describe networks consisting of different subnetworks. For a global perspective it is essential to model also connections between different subsystems in a flexible way. The combination of finitely many types and a continuum of possible weights does exactly the job of delivering realistic network skeletons on which default contagion processes can still be described by finite dimensional fixed point equations. This idea was developed in [17] for a pure default contagion analysis. 
The empirical distribution of parameters (weights and types) is assumed to be close in distribution to a multidimensional random vector $\left(\boldsymbol{W}^{-}, \boldsymbol{W}^{+}, A\right)$.

2. The parameter vector $\left(\boldsymbol{W}^{-}, \boldsymbol{W}^{+}, A\right)$ is then complemented by random components describing the empirical distribution of systemic importance, capital, exogenous loss and asset holdings. This leads to $\left(\boldsymbol{W}^{-}, \boldsymbol{W}^{+}, \boldsymbol{X}, S, C, L, A\right)$ as the parameter of our financial system. We only pose a first moment restriction on $\left(\boldsymbol{W}^{-}, \boldsymbol{W}^{+}, \boldsymbol{X}, S, C, L, A\right)$ and in particular allow for all kind of possible dependencies between the components. We then consider a whole ensemble of financial systems with parameters close in distribution to $\left(\boldsymbol{W}^{-}, \boldsymbol{W}^{+}, \boldsymbol{X}, S, C, L, A\right)$.

For the stochastic system we derive similar results as for the explicit system about the fraction of finally defaulted institutions and the vector of finally sold asset shares. The following informal statement summarises the main insights from Theorem 3.3.

Mock theorem Consider a financial system of size $n \in \mathbb{N}$ that was hit by some exogenous shock. Let $M \in \mathbb{N}$ denote the number of assets in the system. Then under certain regularity assumptions there exist constants $0 \leq k_{0} \leq K_{0} \leq 1$ and $0 \leq k_{m} \leq K_{m}<\infty, 1 \leq m \leq M$, such that

$$
\begin{aligned}
& k_{0}+o_{p}(1) \leq \frac{\text { number of finally defaulted institutions }}{n} \leq K_{0}+o_{p}(1), \\
& k_{m}+o_{p}(1) \leq \frac{\text { number of finally sold shares of asset } m}{n} \leq K_{m}+o_{p}(1),
\end{aligned}
$$

where $o_{p}(1)$ denotes a term that vanishes as $n$ becomes large. The constants $k_{0}, K_{0}, k_{m}, K_{m}$ can be computed explicitly in terms of the global system statistics, and in many relevant settings $k_{0}=K_{0}, k_{m}=K_{m}, m \leq M$.

For large systems we thus derive explicit lower and upper bounds for the fraction of finally defaulted institutions and sold shares triggered by some initial shock. Moreover, the upper and lower bounds coincide in most cases and then our results allow to determine the quantities exactly. The bounds are robust to local changes and are thus suitable to understand the effect of certain structural characteristics of the network and in particular the joint impact of default contagion and fire sales on system stability.

An asymptotic analysis is justifiable by the fact that a large number of institutions (funds, investment banks, insurance companies, etc.) are participating in the financial system. Additionally we test the analytic results with a simulation study, which shows that systems of reasonable size are well approximated by our analytic results.

For the proofs we can partly resort to results from [16-18]. Still the combination of both channels poses a number of new technical challenges. In the pure contagion model one can use so called finitary systems, i.e. systems where the characterizing parameter vector in Item 2. above only takes finitely many values, to approximate default contagion in more general systems (see $[16,17,19]$ ). These finitary systems can be well understood by means of the differential equation method (see [35]). However, once fire sales are included, finitary systems pose some discontinuity issues and thus require new techniques, which we develop here.

Risk management As already mentioned before, our asymptotic approach entails the advantage of a natural notion of resilience. More particularly, we consider arbitrarily small initial shocks and use our main result for the stochastic model to determine whether the resulting fraction of finally defaulted institutions vanishes or remains bounded away from 0 . We derive explicit criteria for regulators to determine the stability of a financial system and we demon- 
strate in an example that resilience can heavily depend on the joint impact of default contagion and fire sales. This specifically underlines the importance of an integrated modelling.

Moreover, based on the resilience criteria we show for a class of financial systems how to determine sufficient capital requirements to ensure stability. The regulated institutions themselves can easily calculate these capital requirements after a regulator announces a few global parameters - they are thus very transparent. Further, as the capital requirements only depend on global parameters and the bank's own business decisions (its balance sheet) they are inherently fair and cannot be manipulated.

\subsection{Outline}

In Sect. 2, we state our model for the financial system and the contagion process. We then state and explain our main result for the stochastic model in Sect. 3. In Sect. 4, we derive criteria for financial networks to be (non-)resilient with respect to small initial shocks. We then apply them to obtain sufficient capital requirements in Sect. 5 and carry out a simulation study to show that our results are applicable for large finite networks and to demonstrate the joint impact of default contagion and fire sales. In Sect. 6 we discuss estimation of the model before we conclude with a discussion including the limitations of the model in Sect. 7. In Sect. 8, we provide proofs for all the results. Finally, we provide a glossary listing all important symbols, parameters and functions for our model.

\section{An integrated model for the financial system}

In this section, we state our model for a financial system. It includes all the parameters we need to investigate the interplay of the contagion channels fire sales and default contagion. We assume that there are $n \in \mathbb{N}$ financial institutions. We use the term financial institutions in a wide sense. It may include banks, insurance companies, mutual funds, asset managers but also non-financial institutions, as for example corporations if they hold a large number of the assets and would sell them in case of a decline in value. We denote the set of institutions by $[n]:=\{1, \ldots, n\}$. Further we consider $M \in \mathbb{N}$ assets. These are the assets institutions invest in and that are considered relevant for potential fire sales. We denote by $[M]:=\{1, \ldots, M\}$ the set of these assets.

\subsection{Model parameters}

Each institution $i \in[n]$ has a set of parameters assigned:

1. The value of systemic importance $s_{i} \in \mathbb{R}_{+, 0}$ : It describes the potential damage that a default of institution $i$ will cause for the global financial system or the wider economy. For example, if institution $i \in[n]$ is crucial in providing certain payment infrastructure it would have a higher value $s_{i}$ assigned than a bank that is not offering such service. See [19] for more details and possible choices for $s_{i}$.

2. The initial capital parameter $c_{i} \in \mathbb{R}_{+, \infty}:=\mathbb{R}_{+} \cup\{\infty\}$ : This parameter determines the monetary buffer of institution $i$ against losses. For banks this is usually their equity (assets minus liabilities), which is positive if the bank is solvent. For low leverage institutions, such as mutual and money market funds, it could the total value of assets managed. In the following, we refer to $c_{i}$ as capital for simplicity. 
Fig. 1 Model parameters for institutions $i$ and $j$ in the financial system as well as asset $m$

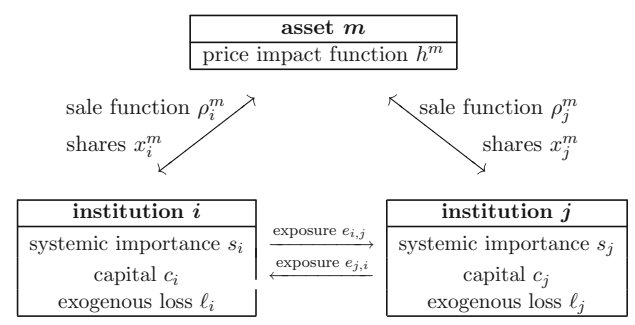

3. The exogenous loss parameter $\ell_{i} \in \mathbb{R}_{+, 0}$ : It models the impact of some external shock on institution $i$. The specification of $\ell_{i}$ allows for a variety of stress tests for the financial system, i.e. asset price shock, defaults of some institutions, etc. The actual magnitude of $\ell_{i}$ will thus crucially depend on the stress testing and the business model of institution $i$. The actual new capital of institution $i$ after the shock is $c_{i}-\ell_{i}$.

4. The number $x_{i}^{m} \in \mathbb{R}_{+, 0}$ of shares institution $i$ holds of asset $m \in[M]$ : As we are only interested in the effect of sales, we consider only positive holdings. If an institution $i$ is shortening asset $m$, we set $x_{i}^{m}=0$. So for each institution we can assign a vector $\boldsymbol{x}_{i}:=\left(x_{i}^{1}, \ldots, x_{i}^{M}\right) \in \mathbb{R}_{+, 0}^{M}$ of asset holdings.

5. Direct exposures $e_{i, j} \in \mathbb{R}_{+, 0}$ : In this section, we consider $e_{i, j}$ to be the observed (deterministic) exposure of $j$ to $i$. If $e_{i, j}>0$, this means that institution $i$ owes a monetary amount to institution $j$ via for example a loan. In the next section, we will propose a random model for $\left\{e_{i, j}\right\}_{i, j \in[n] \text {. }}$

6. A sales function $\rho_{i}: \mathbb{R}_{+, 0} \rightarrow[0,1]^{M}$ : This function models the sales of asset $1, \ldots, M$ by institution $i$ after it lost a certain fraction of its capital.

In Fig. 1 we summarize the parameters assigned to each institution.

\subsection{Fire sales}

Fire sales are the combination of asset sales and price impact. The exogenous losses $\ell_{i}, i \in[n]$, possibly drive some institutions into selling parts of their assets. This can be due to their own risk preference or regulation that forces them to stay within certain risk bounds or leverage constraints. We model these asset sales by vector valued functions $\rho_{i}=\left(\rho_{i}^{1}, \ldots, \rho_{i}^{M}\right)$ : $\mathbb{R}_{+, 0} \rightarrow[0,1]^{M}$, which describe the fraction of holdings of asset $1, \ldots, M$ sold by institution $i$ after it lost a certain fraction of its capital, i.e. $i \in[n]$ sells $x_{i}^{m} \rho_{i}^{m}\left(\Lambda / c_{i}\right)$ of its shares of asset $m$ after it incurred losses of $\Lambda$. The specification of $\rho_{i}$ will crucially depend on the type of institution (i.e. asset manager, investment bank, insurance company,...) and the respective regulatory requirement. Allowing for different sales behavior for different institutions is thus especially important if one goes beyond the banking network. Moreover, by allowing $\rho_{i}$ to be vector valued, we account for the fact that financial institutions might follow a certain strategy when selling assets. For example they might start with liquid assets first or try to keep their overall market exposure. We make the following natural assumptions: the sale functions $\rho_{i}$ for $i \in[n]$ are non-decreasing, $\rho_{i}(0)=(0, \ldots, 0)$ and $\rho_{i}(u)=(1, \ldots, 1)$ for all $u \geq 1$. Moreover to simplify notation in the following, we choose $\rho_{i}$ to be right-continuous for every $i \in[n]$ and denote by $\stackrel{\circ}{\rho}_{i}(u):=\lim _{\epsilon \rightarrow 0+} \rho_{i}((1-\epsilon) u)$ its left-continuous modification.

The sales will cause prices to go down because the assets are not perfectly liquid (the limit order book has finite depth). To model the decline in the asset prices we use functions $h^{m}: \mathbb{R}_{+, 0}^{M} \rightarrow[0,1]$ which are non-decreasing and continuous in each coordinate. We 
assume that the share price of each asset $m \in[M]$ decreases by $h^{m}(\boldsymbol{y})$ after $n y^{m} \in \mathbb{R}_{+, 0}$ shares of asset $m$ have been sold, where $\boldsymbol{y}=\left(y^{1}, \ldots, y^{M}\right)$. Each institution $i \in[n]$ is further assumed to suffer losses of $\boldsymbol{x}_{i} \cdot h(\boldsymbol{y})$ due to mark-to-market valuation of its portfolio, where $h(\boldsymbol{y})=\left(h^{1}(\boldsymbol{y}), \ldots, h^{M}(\boldsymbol{y})\right)$.

There are two remarks in order: First, we pick $\boldsymbol{y}$ as the argument of $h$ instead of the actual vector of sold shares $n \boldsymbol{y}$; this choice is purely conventional for any fixed $n$ but will be convenient for our results (also cf. Assumption 3.1). Second, as institutions start selling assets during the contagion process, they actually reduce their exposure to future price drops and $\boldsymbol{x}_{i} \cdot h(\boldsymbol{y})$ merely functions as an upper bound on $i$ 's losses. In this sense our model is conservative, and moreover, this assumption allows for better analytic results in the following.

For a financial system without direct exposures, i.e. $e_{i, j}=0$ for all $i, j \in[n]$, the contagion process is then solely driven by rounds of alternating asset sales and price impact, i.e. fire sales. Denoting by $\boldsymbol{\sigma}_{(k)}=\left(\sigma_{(k)}^{1}, \ldots, \sigma_{(k)}^{M}\right)$ the vector of sold shares in round $k \in \mathbb{N}_{0}$ with $\sigma_{(0)}=\mathbf{0}$ we then derive

$$
\boldsymbol{\sigma}_{(k)}=\sum_{i \in[n]} \boldsymbol{x}_{i} \odot \rho_{i}\left(\frac{\ell_{i}+\boldsymbol{x}_{i} \cdot h\left(n^{-1} \boldsymbol{\sigma}_{(k-1)}\right)}{c_{i}}\right), \quad k \geq 1 .
$$

Here $\odot$ denotes the Hadamard (entry-wise) product of two vectors, while $\cdot$ denotes the scalar product. See [18] for results on the pure fire sales process in a slightly more restricted setting.

\subsection{Default contagion}

If on the other hand, we consider a financial system with $\boldsymbol{x}_{i}=\mathbf{0}$ for all $i \in[n]$, then contagion completely proceeds via the direct exposure network. That is, if $\ell_{i} \geq c_{i}$ and institution $i \in[n]$ is therefore initially defaulted, then each institution $j \in[n]$ suffers losses of $e_{i, j}$. This possibly causes further defaults in the system and so on. Note that we suppose a recovery rate of zero, which is a conservative yet reasonable assumption because the time horizon of the default contagion process is short compared to the time the resolution of an insolvent institution takes, and there is a huge amount of uncertainty about the actual value of an insolvent institution immediately after its default. One could easily implement other fixed recovery rates in our model.

Again we can consider the pure default contagion process in rounds. Denoting $\mathcal{D}_{(k)} \subseteq[n]$ the set of defaulted institutions in round $k \in \mathbb{N}_{0}$ with $\mathcal{D}_{(0)}:=\left\{i \in[n]: 0 \geq c_{i}-\ell_{i}\right\}$ we obtain

$$
\mathcal{D}_{(k)}=\left\{i \in[n]: \sum_{j \in \mathcal{D}_{(k-1)}} e_{j, i} \geq c_{i}-\ell_{i}\right\}, \quad k \geq 1,
$$

and the contagion process ends after at most $n-1$ rounds. In particular, $\mathcal{D}_{n}:=\mathcal{D}_{(n-1)}$ consists of all finally defaulted institutions and $\mathcal{S}_{n}=\sum_{i \in \mathcal{D}_{n}} s_{i}$ amounts to the total final systemic damage caused by defaults. See $[3,16,17]$ for more results on the pure default contagion process.

\subsection{The contagion process}

The focus of this section is on the understanding of the joint effects of fire sales and default contagion. We therefore combine the two processes from above and again consider contagion in rounds: Let $\mathcal{D}_{(0)}=\emptyset$ and $\boldsymbol{\sigma}_{(0)}=\mathbf{0} \in \mathbb{R}^{M}$. Moreover, for $k \geq 1$, 


$$
\mathcal{D}_{(k)}=\left\{i \in[n]: \sum_{j \in \mathcal{D}_{(k-1)}} e_{j, i} \geq c_{i}-\ell_{i}-\boldsymbol{x}_{i} \cdot h\left(n^{-1} \boldsymbol{\sigma}_{(k-1)}\right)\right\}
$$

and

$$
\boldsymbol{\sigma}_{(k)}=\sum_{i \in[n]} \boldsymbol{x}_{i} \odot \rho_{i}\left(\frac{\sum_{j \in \mathcal{D}_{(k-1)}} e_{j, i}+\ell_{i}+\boldsymbol{x}_{i} \cdot h\left(n^{-1} \boldsymbol{\sigma}_{(k-1)}\right)}{c_{i}}\right) .
$$

Then $\mathcal{D}_{(0)} \subseteq \mathcal{D}_{(1)} \subseteq \cdots \subseteq[n]$ and $\boldsymbol{\sigma}_{(0)} \leq \boldsymbol{\sigma}_{(1)} \leq \cdots \leq \sum_{i \in[n]} \boldsymbol{x}_{i}$. We can thus conclude that the process converges as $k \rightarrow \infty$. Let then $\mathcal{D}_{n}:=\bigcup_{k \in \mathbb{N}} \mathcal{D}_{(k)}$ be the set of finally defaulted institutions, $\mathcal{S}_{n}=\sum_{i \in \mathcal{D}_{n}} s_{i}$ their systemic importance and $\chi_{n}:=\mathrm{n}^{-1} \lim _{k \rightarrow \infty} \sigma_{(k)}$ the vector of finally sold shares divided by $n$.

Lemma 2.1 There exists a smallest solution $\left(\overline{\mathcal{D}}_{n}, \bar{\chi}_{n}\right)$ to

$$
\begin{aligned}
& \mathcal{D}=\left\{i \in[n]: \sum_{j \in \mathcal{D}} e_{j, i} \geq c_{i}-\ell_{i}-\boldsymbol{x}_{i} \cdot h(\chi)\right\}, \\
& \chi=n^{-1} \sum_{i \in[n]} \boldsymbol{x}_{i} \odot \rho_{i}\left(\frac{\sum_{j \in \mathcal{D}} e_{j, i}+\ell_{i}+\boldsymbol{x}_{i} \cdot h(\chi)}{c_{i}}\right) .
\end{aligned}
$$

Moreover, $\mathcal{D}_{n} \subseteq \overline{\mathcal{D}}_{n}$ and $\chi_{n} \leq \bar{\chi}_{n}$.

The case that $\mathcal{D}_{n} \subsetneq \overline{\mathcal{D}}_{n}$ or $\chi_{n}<\bar{\chi}_{n}$ can happen if in the contagion process the sold shares converge to a vector that would be large enough to cause new defaults or trigger further asset sales but is actually never reached in finitely many steps. Then the process converges to a non-equilibrium state. As for real financial systems the least possible number of sold shares in each round is lower bounded by 1 , this can actually never happen and for all practical purposes the final set of defaulted institutions is given by $\overline{\mathcal{D}}_{n}$, their caused systemic damage by $\overline{\mathcal{S}}_{n}:=\sum_{i \in \overline{\mathcal{D}}_{n}} s_{i}$ and the vector of finally sold shares is given by $\bar{\chi}_{n}$.

Furthermore, in the next section the following explicit lower bound on $\left(\mathcal{D}_{n}, \chi_{n}\right)$ will be necessary to draw an extensive picture of the contagion process.

Lemma 2.2 Let $\stackrel{\circ}{\rho}_{i}$ be the left-continuous modification of $\rho_{i}$ for every $i \in[n]$. Then there exists a smallest solution $\left(\hat{\mathcal{D}}_{n}, \hat{\chi}_{n}\right)$ to

$$
\begin{aligned}
& \mathcal{D}=\left\{i \in[n]: \sum_{j \in \mathcal{D}} e_{j, i}>c_{i}-\ell_{i}-\boldsymbol{x}_{i} \cdot h(\chi)\right\}, \\
& \chi=n^{-1} \sum_{i \in[n]} \boldsymbol{x}_{i} \odot \stackrel{\circ}{\rho}_{i}\left(\frac{\sum_{j \in \mathcal{D}} e_{j, i}+\ell_{i}+\boldsymbol{x}_{i} \cdot h(\chi)}{c_{i}}\right) .
\end{aligned}
$$

Moreover, $\hat{\mathcal{D}}_{n} \subseteq \mathcal{D}_{n}$ and $\hat{\chi}_{n} \leq \chi_{n}$.

Finally, denote $\hat{\mathcal{S}}_{n}=\sum_{i \in \hat{\mathcal{D}}_{n}} s_{i}$. Then altogether, we derive the following statement.

Proposition 2.3 For the set of finally defaulted institutions $\mathcal{D}_{n}$, their total systemic importance $\mathcal{S}_{n}$ and the vector $\chi_{n}$ of finally sold shares divided by $n$ it holds

$$
\hat{\mathcal{D}}_{n} \subseteq \mathcal{D}_{n} \subseteq \overline{\mathcal{D}}_{n}, \quad \hat{\mathcal{S}}_{n} \leq \mathcal{S}_{n} \leq \overline{\mathcal{S}}_{n}, \quad \hat{\chi}_{n} \leq \chi_{n} \leq \bar{\chi}_{n} .
$$




\section{The stochastic model}

In the previous section, we considered the combined contagion process of fire sales and default contagion on any explicitly given financial system. In this section, we go one step further and analyze a whole ensemble of systems simultaneously that share certain statistical characteristics, i.e. we introduce a random model for the financial system that captures the essential characteristics observed for real financial systems. This will ultimately help us to understand which system structures promote global contagion or contain it locally.

In a first step we specify a random model for the inter-institutional exposures $e_{i, j}$; we adopt the multi-type financial network model from [17]. We let the connection probability of two institutions in the system depend on their respective types-larger within communities or within cores, smaller between communities and for periphery institutions for instance. Second, we allow for different exposure distributions between different institution typeslarger exposures between core-institutions for example.

From now on we assume that inter-institutional exposures $e_{i, j}$ are bounded and take integer values in $\{0, \ldots, R\}$ for some $R \in \mathbb{N}$. We refer to [31] for an extension to exposures modelled by sequences of exchangeable (possibly unbounded) random variables in the multitype financial network model. We define a probability measure $\mathbb{P}$ on $\{0, \ldots, R\}^{|E|}$, where $E$ is the set of possible directed edges $E:=\left\{(i, j) \in[n]^{2}: i \neq j\right\}$. To model random exposures has several advantages:

1. The network of exposures can change significantly on a microscopic level but as empirical studies show, the global statistics are reasonably stable (see e.g. [14]).

2. Often only the aggregated exposures $\sum_{j \in[n]} e_{i, j}$ are available to the regulator. Since the individual exposures are unknown it is thus advisable to use the information available and consider probabilistic samples. Ideally one obtains results that hold for all possible realizations.

3. A random network is analytically more tractable and provides more understanding of the impact of the network characteristics on the combined fire sales and contagion process.

Our choice of $\mathbb{P}$ has to be such that the generated networks share the characteristics of observed financial networks. We assume that the global financial system is composed of institutions of $T \in \mathbb{N}$ different types in total. Instead of assuming deterministic exposures $\left\{e_{i, j}\right\}_{j \in[n]} \subset \mathbb{R}_{+, 0}$ as given Item 5. in Sect. 2.1 we now associate to each institution $i \in[n]$ :

5'. (a) An institution-type $\alpha_{i} \in[T]$ : This parameter allocates institution $i$ to a certain subsystem such as country or core/periphery.

$5^{\prime}$. (b) A vector of in-weights $\boldsymbol{w}_{i}^{-} \in \mathbb{R}_{+, 0}^{[R] \times[T]}$ : The in-weight $w_{i}^{-, r, \alpha}$ describes the tendency of institution $i$ to be exposed to an institution of type $\alpha$ with an exposure of size $r$.

$5^{\prime}$. (c) A vector of out-weights $\boldsymbol{w}_{i}^{+} \in \mathbb{R}_{+, 0}^{[R] \times[T]}$ : The out-weight $w_{i}^{+, r, \alpha}$ describes the tendency of institutions of type $\alpha$ to be exposed to $i$ with an exposure of size $r$.

We assume in the following that institutions of the same type have the same sales function. This still allows to distinguish between different types such as e.g. country, core/periphery, banks/insurance companies/wealth managers, combinations of the aforementioned, or any other reasonable segmentation and as noted before is important to account for different regulatory environments. We denote by $\rho_{\alpha}$ the sales function of institutions of type $\alpha$. The occurrence of an edge of multiplicity $r \in[R]$ (exposure size) going from $i$ to $j$ is then modeled by a Bernoulli random variable $X_{i, j}^{r}$ with success probability 


$$
p_{i, j}^{r}:= \begin{cases}\min \left\{R^{-1}, n^{-1} w_{i}^{+, r, \alpha_{j}} w_{j}^{-, r, \alpha_{i}}\right\}, & \text { if } i \neq j, \\ 0, & \text { if } i=j,\end{cases}
$$

where we assume mutual exclusiveness $\left(\sum_{r \in[R]} X_{i, j}^{r} \leq 1\right)$ respectively independence $X_{i_{1}, j_{1}}^{r_{1}} \perp X_{i_{2}, j_{2}}^{r_{2}}$ for all $r_{1}, r_{2}$ and $\left(i_{1}, j_{1}\right) \neq\left(i_{2}, j_{2}\right)$.

Now consider a collection of financial systems with varying size $n$. We want to ensure that their statistical characteristics measured by means of the empirical distribution functions stabilize as $n \rightarrow \infty$. Moreover, we want to prohibit that exposures or asset holdings condense in one institution.

Assumption 3.1 (Regular vertex sequence) Let $M \in \mathbb{N}$. For each $n \in \mathbb{N}$ consider a system with $n$ institutions and $M$ assets specified by the sequences $\boldsymbol{w}^{-}(n)=\left(\boldsymbol{w}_{i}^{-}(n)\right)_{i \in[n]}$ of inweights, $\boldsymbol{w}^{+}(n)=\left(\boldsymbol{w}_{i}^{+}(n)\right)_{i \in[n]}$ of out-weights, $\boldsymbol{x}(n)=\left(\boldsymbol{x}_{i}(n)\right)_{i \in[n]}$ of asset holdings, $\boldsymbol{s}(n)=\left(s_{i}(n)\right)_{i \in[n]}$ of systemic importance values, $\boldsymbol{c}(n)=\left(c_{i}(n)\right)_{i \in[n]}$ of capitals, $\boldsymbol{\ell}(n)=$ $\left(\ell_{i}(n)\right)_{i \in[n]}$ of exogenous losses and $\alpha(n)=\left(\alpha_{i}(n)\right)_{i \in[n]}$ of institution types. Then the following shall hold:

(a) Convergence in distribution: For each $n \in \mathbb{N}$ let the random empirical distribution function of the system parameters be denoted by

$$
\begin{aligned}
& F_{n}\left(\boldsymbol{w}^{-}, \boldsymbol{w}^{+}, \boldsymbol{x}, s, c, \ell, \alpha\right) n^{-1} \sum_{i \in[n]} \prod_{r \in[R], \beta \in[T]} \mathbf{1}\left\{w_{i}^{-r, \beta} \leq w^{-, r, \beta}, w_{i}^{+, r, \beta} \leq w^{+, r, \beta}\right\} \\
& \quad \times \prod_{m \in[M]} \mathbf{1}\left\{x_{i}^{m} \leq x^{m}\right\} \mathbf{1}\left\{s_{i} \leq s, c_{i} \leq c, \ell_{i} \leq \ell, \alpha_{i} \leq \alpha\right\},
\end{aligned}
$$

for $\boldsymbol{w}^{-}, \boldsymbol{w}^{+} \in \mathbb{R}_{+, 0}^{[R] \times[T]}, \boldsymbol{x} \in \mathbb{R}_{+, 0}^{M}, s \in \mathbb{R}_{+, 0}, c \in \mathbb{R}_{+, 0, \infty}, \ell \in \mathbb{R}_{+, 0}$ and $\alpha \in[T]$. Let in the following $\left(\boldsymbol{W}_{n}^{-}, \boldsymbol{W}_{n}^{+}, \boldsymbol{X}_{n}, S_{n}, C_{n}, L_{n}, A_{n}\right)$ denote a random vector distributed according to $F_{n}$. Then there exists a distribution function $F$ such that

$$
F_{n}\left(\boldsymbol{w}^{-}, \boldsymbol{w}^{+}, \boldsymbol{x}, s, c, \ell, \alpha\right) \rightarrow F\left(\boldsymbol{w}^{-}, \boldsymbol{w}^{+}, \boldsymbol{x}, s, c, \ell, \alpha\right), \quad \text { as } n \rightarrow \infty,
$$

at all continuity points of $F_{\alpha}\left(\boldsymbol{w}^{-}, \boldsymbol{w}^{+}, \boldsymbol{x}, s, c, \ell\right):=F\left(\boldsymbol{w}^{-}, \boldsymbol{w}^{+}, \boldsymbol{x}, s, c, \ell, \alpha\right)$.

(b) Convergence of means: Denote by $\left(\boldsymbol{W}^{-}, \boldsymbol{W}^{+}, \boldsymbol{X}, S, C, L, A\right)$ a random vector distributed according to the limiting distribution $F$. Then as $n \rightarrow \infty$,

$$
\begin{aligned}
& \mathbb{E}\left[W_{n}^{-, r, \alpha}\right] \rightarrow \mathbb{E}\left[W^{-, r, \alpha}\right]<\infty, \quad \mathbb{E}\left[W_{n}^{+, r, \alpha}\right] \rightarrow \mathbb{E}\left[W^{+, r, \alpha}\right]<\infty, \quad \text { for all } r \in[R], \alpha \in[T], \\
& \mathbb{E}\left[S_{n}\right] \rightarrow \mathbb{E}[S]<\infty \text { and } \mathbb{E}\left[X_{n}^{m}\right] \rightarrow \mathbb{E}\left[X^{m}\right]<\infty, \text { for all } m \in[M] .
\end{aligned}
$$

Let $V=[R] \times[T]^{2}$. Define now for $z \in \mathbb{R}_{+, 0}^{V}$ and $\chi \in \mathbb{R}_{+, 0}^{M}$,

$$
\begin{aligned}
g(z, \chi) & :=\sum_{\beta \in[T]} \mathbb{E}\left[S \psi\left(Y_{1, \beta}, \ldots, Y_{R, \beta} ; C-L-\boldsymbol{X} \cdot h(\chi)\right) \mathbf{1}\{A=\beta\}\right], \\
f^{r, \alpha, \beta}(z, \chi):= & \mathbb{E}\left[W^{+, r, \alpha} \psi\left(Y_{1, \beta}, \ldots, Y_{R, \beta} ; C-L-\boldsymbol{X} \cdot h(\chi)\right) \mathbf{1}\{A=\beta\}\right] \\
& -z^{r, \alpha, \beta},(r, \alpha, \beta) \in V,
\end{aligned}
$$




$$
\begin{aligned}
f^{m}(z, \chi):= & \sum_{\beta \in[T]} \mathbb{E}\left[X^{m} \phi_{\beta}^{m}\left(Y_{1, \beta}, \ldots, Y_{R, \beta} ; L+X \cdot h(\chi), C\right) \mathbf{1}\{A=\beta\}\right] \\
& -\chi^{m}, m \in[M]
\end{aligned}
$$

where we abbreviate

$$
Y_{r, \beta}:=\sum_{\gamma \in[T]} W^{-, r, \gamma} z^{r, \beta, \gamma}, \quad r \in[R], \beta \in[T],
$$

and for $\left\{q_{s}\right\}_{s \in[R]}$ in $\mathbb{R}_{+, 0}$ and independent $Q_{s} \sim \operatorname{Poi}\left(q_{s}\right), s \in[R]$,

$$
\psi\left(q_{1}, \ldots, q_{R} ; t\right):=\mathbb{P}\left(\sum_{s \in[R]} s Q_{s} \geq t\right),
$$

respectively the vector

$$
\phi_{\alpha}\left(q_{1}, \ldots, q_{R} ; \ell, c\right):=\mathbb{E}\left[\rho_{\alpha}\left(\frac{\sum_{s \in[R]} s Q_{s}+\ell}{c}\right)\right], \quad \alpha \in[T] .
$$

Let us give an intuitive explanation for the functions $g, f^{r, \alpha, \beta}$ and $f^{m}$ first. For this we consider the special case $R=T=1$ and start by looking at the fire sales and the default contagion process separately.

We start with the default contagion process. Heuristically, for an externally given vector of asset sales $\chi$, the function $f^{1,1,1}(\cdot, \chi)$ describes (in the limit $n \rightarrow \infty$ ) the intensity of the default contagion process over time. Here time refers to steps in a sequential analysis of the process, which leads to the same set of defaulted institutions. Let now $\bar{z} \in\left[0, \mathbb{E}\left[W^{+}\right]\right]$ denote the total out-weight of finally defaulted banks divided by $n$. Then by the specification of $p_{i, j}$ for any fixed bank $i \in[n]$ the number of incoming edges (exposures) from finally defaulted banks is given by a random variable $\operatorname{Poi}\left(w_{i}^{-, 1,1} \bar{z}\right)$. Institution $i$ is hence finally defaulted itself if and only if $\operatorname{Poi}\left(w_{i}^{-, 1,1} \bar{z}\right) \geq c_{i}-\ell_{i}-\boldsymbol{x}_{\boldsymbol{i}} \cdot h(\chi)$. Summing over all banks in the system we thus derive the following identity:

$$
\begin{aligned}
\bar{z} & =n^{-1} \sum_{i \in[n]} w_{i}^{+, 1,1} \mathbf{1}\left\{\operatorname{Poi}\left(w_{i}^{-, 1,1} \bar{z}\right) \geq c_{i}-\ell_{i}-\boldsymbol{x}_{i} \cdot h(\chi)\right\} \\
& \approx \mathbb{E}\left[W^{+, 1,1} \psi\left(W^{-, 1,1} \bar{z} ; C-L-\boldsymbol{X} \cdot h(\chi)\right)\right],
\end{aligned}
$$

and therefore $f^{1,1,1}(\bar{z}, \chi)=0$. Further, the damage by finally defaulted banks is then given by

$$
n^{-1} \sum_{i \in[n]} s_{i} \mathbf{1}\left\{\ell_{i}+\operatorname{Poi}\left(w_{i}^{-, 1,1} \bar{z}\right)+\boldsymbol{x}_{i} \cdot h(\boldsymbol{\chi}) \geq c_{i}\right\} \approx \mathbb{E}\left[S \psi\left(W^{-, 1,1} \bar{z}, C-L-\boldsymbol{X} \cdot h(\boldsymbol{\chi})\right)\right]=g(\bar{z}, \boldsymbol{\chi}) .
$$

Hence if fire sales are ignored, meaning the initial capital is simply reduced by a fixed amount accounting for some externally given sales vector $\chi$, then in order to get the final state of the system we only need to determine the (first) root $\bar{z}$ of $f^{1,1,1}(\cdot, \chi)$ and plug it into $g(\cdot, \chi)$.

Let us now look at the fire sales system with an externally given default contagion result. For the case of one asset with label 1, fixing the sum of the out-weights of defaulted institutions, divided by $n$ to be $z$, then the loss institution $i$ receives due to the liabilities to defaulted 
banks is described by the random quantity $\operatorname{Poi}\left(w_{i}^{-, 1,1} z\right)$ and, for continuous $\rho_{1}$, similarly as in the derivation of Lemma 2.1, the number of finally sold shares $\bar{\chi}$ solves

$$
\left.\bar{\chi}=n^{-1} \sum_{i \in[n]} x_{i}^{1} \rho_{1}^{1}\left(\frac{\ell_{i}+\operatorname{Poi}\left(w_{i}^{-, 1,1} z\right)+x_{i}^{1} h^{1}(\bar{\chi})}{c_{i}}\right) \approx \mathbb{E}\left[X^{1} \phi_{1}\left(W^{-, 1,1} z ; L+X^{1} h(\bar{\chi}), C\right)\right)\right]
$$

such that $\bar{\chi}$ is a root of $f^{1}(z, \cdot)$. Moreover, the final systemic importance of defaulted institutions divided by $n$ is given by

$$
n^{-1} \sum_{i \in[n]} s_{i} \mathbf{1}\left\{\operatorname{Poi}\left(w_{i}^{-, 1,1} z\right)+\ell_{i}+x_{i}^{1} h^{1}(\bar{\chi}) \geq c_{i}\right\} \approx \mathbb{E}\left[S \psi\left(W^{-, 1,1} z, C-L-X^{1} h(\bar{\chi})\right)\right]=g(z, \bar{\chi}) .
$$

So the root $\bar{\chi}$ of $f^{1}(z, \cdot)$ determines the end of the process and again $g$ yields the damage by defaulted institutions.

These heuristics show that the joint fire sales and default contagion process should come to an end at a joint root of the functions $f^{r, \alpha, \beta},(r, \alpha, \beta) \in V$, and $f^{m}, m \in[M]$. Under some circumstances, however, if the distribution of $\left(\boldsymbol{W}^{-}, \boldsymbol{W}^{+}, \boldsymbol{X}, S, C, L, A\right)$ has atoms and one or more of the functions $\rho_{\alpha}, \alpha \in[T]$, are discontinuous, also the functions $f^{r, \alpha, \beta}$ and $f^{m}$ might be discontinuous. Similar as in the previous section, it is then in general not possible to determine the precise end state of the system. Still we will be able to derive lower bounds on the final default fraction and the vector of finally sold shares. To this end, define lower semi-continuous modifications of $g, f^{r, \alpha, \beta},(r, \alpha, \beta) \in V$, and $f^{m}, m \in[M]$, by

$$
\begin{aligned}
\stackrel{\circ}{g}(z, \chi):= & \sum_{\beta \in[T]} \mathbb{E}\left[S \stackrel{\circ}{\psi}\left(Y_{1, \beta}, \ldots, Y_{R, \beta} ; C-L-\boldsymbol{X} \cdot h(\chi)\right) \mathbf{1}\{A=\beta\}\right], \\
\stackrel{\circ}{f}^{r, \alpha, \beta}(z, \chi):= & \mathbb{E}\left[W^{+, r, \alpha} \stackrel{\circ}{\psi}\left(Y_{1, \beta}, \ldots, Y_{R, \beta} ; C-L-\boldsymbol{X} \cdot h(\chi)\right) \mathbf{1}\{A=\beta\}\right] \\
& -z^{r, \alpha, \beta},(r, \alpha, \beta) \in V, \\
\stackrel{\circ}{f}^{m}(z, \chi):= & \sum_{\beta \in[T]} \mathbb{E}\left[X^{m} \stackrel{\circ}{\phi}_{\beta}^{m}\left(Y_{1, \beta}, \ldots, Y_{R, \beta} ; L+\boldsymbol{X} \cdot h(\chi), C\right) \mathbf{1}\{A=\beta\}\right] \\
& -\chi^{m}, m \in[M],
\end{aligned}
$$

where as before

$$
Y_{r, \beta}:=\sum_{\gamma \in[T]} W^{-, r, \gamma} z^{r, \beta, \gamma}, \quad r \in[R], \beta \in[T],
$$

and for $\left\{q_{s}\right\}_{s \in[R]}$ in $\mathbb{R}_{+, 0}$ and independent $Q_{s} \sim \operatorname{Poi}\left(q_{s}\right), s \in[R]$,

$$
\stackrel{\circ}{\psi}\left(q_{1}, \ldots, q_{R} ; t\right):=\mathbb{P}\left(\sum_{s \in[R]} s Q_{s}>t\right),
$$

and the vector

$$
\stackrel{\circ}{\phi}_{\beta}\left(q_{1}, \ldots, q_{R} ; \ell, c\right):=\mathbb{E}\left[\stackrel{\circ}{\rho}_{\beta}\left(\frac{\sum_{s \in[R]} s Q_{s}+\ell}{c}\right)\right], \quad \beta \in[T],
$$


for $\stackrel{\circ}{\rho}_{\beta}(u):=\lim _{\epsilon \rightarrow 0+} \rho_{\beta}((1-\epsilon) u)$. Further, let $\stackrel{\circ}{P}_{0}$ and $P_{0}$ the largest connected subsets of

$$
\begin{aligned}
\stackrel{\circ}{P}:= & \bigcap_{(r, \alpha, \beta) \in V}\left\{(z, \chi) \in \mathbb{R}_{+, 0}^{V} \times \mathbb{R}_{+, 0}^{M}: \stackrel{\circ}{f}^{r, \alpha, \beta}(z, \chi) \geq 0\right\} \\
& \cap \bigcap_{m \in[M]}\left\{(z, \chi) \in \mathbb{R}_{+, 0}^{V} \times \mathbb{R}_{+, 0}^{M}: \stackrel{\circ}{f}^{m}(z, \chi) \geq 0\right\}
\end{aligned}
$$

respectively

$$
\begin{aligned}
P:= & \bigcap_{(r, \alpha, \beta) \in V}\left\{(z, \chi) \in \mathbb{R}_{+, 0}^{V} \times \mathbb{R}_{+, 0}^{M}: f^{r, \alpha, \beta}(z, \chi) \geq 0\right\} \\
& \cap \bigcap_{m \in[M]}\left\{(z, \chi) \in \mathbb{R}_{+, 0}^{V} \times \mathbb{R}_{+, 0}^{M}: f^{m}(z, \chi) \geq 0\right\}
\end{aligned}
$$

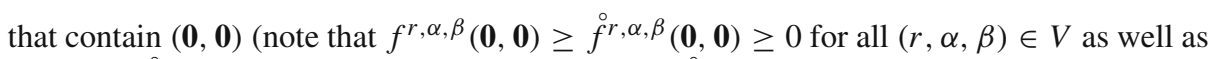
$f^{m}(\mathbf{0}) \geq \stackrel{\circ}{f}^{m}(\mathbf{0}) \geq 0$ for all $m \in[M]$ and thus $\mathbf{0} \in \stackrel{\circ}{P}$ and $\mathbf{0} \in P$ ). We will later make use of the fact that $P$ and $P_{0}$ are clearly closed sets. Finally, define $z^{*} \in \mathbb{R}_{+, 0}^{V}$ and $\chi^{*} \in \mathbb{R}_{+, 0}^{M}$ by $\left(z^{*}\right)^{r, \alpha, \beta}:=\sup _{(z, \chi) \in P_{0}} z^{r, \alpha, \beta}$ and $\left(\chi^{*}\right)^{m}:=\sup _{(z, \chi) \in P_{0}} \chi^{m}$. Then the following holds:

Lemma 3.2 There exists a smallest joint root $(\hat{z}, \hat{\chi})$ of all the functions $\stackrel{\circ}{f}^{r, \alpha, \beta},(r, \alpha, \beta) \in V$, and $\stackrel{\circ}{f}^{m}, m \in[M]$. It holds $(\hat{z}, \hat{\chi}) \in \stackrel{\circ}{P}_{0}$. Further, $\left(z^{*}, \chi^{*}\right)$ as defined above is a joint root of the functions $f^{r, \alpha, \beta}, f^{m}$ and $\left(z^{*}, \chi^{*}\right) \in P_{0}$.

The proof is analogue to the one of [18, Lemma EC. 1]. We can then describe the final default fraction and the number of sold shares asymptotically as $n \rightarrow \infty$ in terms of $(\hat{z}, \hat{\chi})$ and $\left(z^{*}, \chi^{*}\right)$.

Theorem 3.3 Consider a financial system that fulfills Assumption 3.1. Then for the final systemic damage $n^{-1} \mathcal{S}_{n}$ and $\chi_{n}^{m}$, the number of finally sold shares of asset $m \in[M]$ divided by $n$, it holds

$$
\begin{aligned}
\stackrel{\circ}{g}(\hat{z}, \hat{\chi})+o_{p}(1) & \leq n^{-1} \mathcal{S}_{n} \leq g\left(z^{*}, \chi^{*}\right)+o_{p}(1), \\
\hat{\chi}^{m}+o_{p}(1) & \leq \chi_{n}^{m} \leq\left(\chi^{*}\right)^{m}+o_{p}(1) .
\end{aligned}
$$

In particular, for the final price impact $h^{m}\left(\chi_{n}\right)$ on asset $m \in[M]$ it holds

$$
h^{m}(\hat{\chi})+o_{p}(1) \leq h^{m}\left(\chi_{n}\right) \leq h^{m}\left(\chi^{*}\right)+o_{p}(1) .
$$

In most cases, $(\hat{z}, \hat{\chi})$ and $\left(z^{*}, \chi^{*}\right)$ will coincide and $\stackrel{\circ}{g}(\hat{z}, \hat{\chi})=g\left(z^{*}, \chi^{*}\right)$. Theorem 3.3 then describes the limits in probability of $\chi_{n}$ and $n^{-1} \mathcal{S}_{n}$ for $n \rightarrow \infty$. Note that the final default fraction can be obtained from the theorem by choosing $S \equiv 1$.

\section{Resilient and non-resilient systems}

Our results from the previous section allow us to compute the final state of a system that was initially hit by some exogenous shock starting a cascade of default contagion and fire sales. We shall now go one step further and describe the vulnerability of an initially unshocked system to small shocks. We achieve this goal by considering shocks $L$ of different magnitude on the same initially unshocked system described by $\left(\boldsymbol{W}^{-}, \boldsymbol{W}^{+}, \boldsymbol{X}, S, C, A\right)$. In the following, 
if we use the notations $g, \stackrel{\circ}{g}, z^{*}$ and $\chi^{*}$ from Sect. 3 we mean the unshocked system with $L \equiv 0$.

Our notion of resilience is related to the one for pure fire sales in [18] and this section uses and extends ideas and methods from [18, Sect. 3].

\subsection{Resilience}

When it comes to regulation of a financial system, one desirable property is the capability to absorb local shocks rather than amplify them through large parts of the system. In our asymptotic model we can consider arbitrarily small shocks $L$ and the following natural notion of resilience emerges: when considering initial shocks $L$ such that $\mathbb{E}[L / C] \rightarrow 0$, then a system is called resilient if also the induced asymptotic final damage $n^{-1} \mathcal{S}_{n, L}$ tends to 0 .

Here and in the following we say that a sequence of events $\left(E_{n}\right)_{n \in \mathbb{N}}$ holds with high probability (w.h.p.) if $\mathbb{P}\left(E_{n}\right) \rightarrow 1$, as $n \rightarrow \infty$.

Definition 4.1 (Resilience) A financial system $\left(\boldsymbol{W}^{-}, \boldsymbol{W}^{+}, \boldsymbol{X}, S, C, A\right)$ is said to be resilient, if for each $\epsilon>0$ there exists $\delta>0$ such that for all $L$ with $\mathbb{E}[L / C]<\delta$ it holds $n^{-1} \mathcal{S}_{n, L} \leq \epsilon$ with high probability.

While this definition (and Corollary 4.3) is concerned with the final systemic damage only, the following theorem also investigates the number of sold shares of the assets (and hence the price impacts which also affect the wider economy) in the limit $\mathbb{E}[L / C] \rightarrow 0$.

Theorem 4.2 For each $\epsilon>0$ there exists $\delta>0$ such that for all $L$ with $\mathbb{E}[L / C]<\delta$ the final damage by defaulted institutions $n^{-1} \mathcal{S}_{n, L}$ and the number $n \chi_{n, L}^{m}$ of finally sold shares of asset $m \in[M]$ in the shocked system satisfy w.h.p.

$$
n^{-1} \mathcal{S}_{n, L} \leq g\left(z^{*}, \chi^{*}\right)+\epsilon \text { and } \chi_{n, L}^{m} \leq\left(\chi^{*}\right)^{m}+\epsilon, \quad m \in[M] .
$$

In particular, we derive the following resilience criterion.

Corollary 4.3 (Resilience criterion) If $g\left(z^{*}, \chi^{*}\right)=0$, then the system is resilient.

It is thus sufficient for resilience if $\left(z^{*}, \chi^{*}\right)=(\mathbf{0}, \mathbf{0})$ or equivalently $P_{0}=\{(\mathbf{0}, \mathbf{0})\}$. If, however, $g\left(z^{*}, \chi^{*}\right)=0$ while $\chi^{*} \neq \mathbf{0}$, then Corollary 4.3 still ensures that the final systemic damage stays small and the system is resilient by Definition 4.1, while a large fraction of shares of assets is sold due to fire sales as a reaction to small local shocks-see Theorem 4.6.

Remark 4.4 Let us compare our results with those obtained in combined default contagion and fire-sales analysis in an Eisenberg-Noe setting as for example performed in [5,12,34]: Although there are differences in the details of the contagion process and the exact specification of fire-sales, our deterministic setup in Sect. 2 and our Proposition 2.3 shares similarity with the setup of extended Eisenberg-Noe models and existence results of a greatest and lowest clearing vector in that literature. However, the results in Sect. 3 depart from this setting by analyzing the stochastic model and $n \rightarrow \infty$ limits. Due to the asymptotic analysis one gains (a) a description of the final default set and the number of sold assets as a fixpoint equation of reduced dimension (Theorem 3.3) and (b) a classification of resilience (in particular Theorem 4.2) which is not possible in the deterministic setting. While, our setup ignores possible intervention by regulators, the idea behind our Definition 4.1 of resilience is that the financial system is stable enough such that in case a cascade starts, intervention is not necessary because amplification effects are small. The results serve as a guideline for the design 
of regulatory requirements that ensure that the financial system has the desirable property that intervention is not necessary in case of a shock. This is in contrast to the analysis in [5] where the authors provide an analytical framework to measure the size of the amplification, and thus the conditions under which the threat of a regulator of not intervening is credible.

\subsection{Non-resilience}

We now aim at characterizing non-resilient systems. For this note that our fire sales model used here is in itself a conservative model as for each institution $i \in[n]$ the entire asset holdings $\boldsymbol{x}_{i}$ are exposed to the price impact $h\left(\chi_{n}\right)$. It therefore ignores intermediate sales at a more favorable asset price level. We refer to [18] for more discussion on and a treatment of intermediate sales. The following results still give a first indication of non-resilience for general financial systems.

We consider shocks of the form $\ell_{i} \in\left\{0,2 c_{i}\right\}$ such that $\mathbb{P}(L=2 C)>0$ and $L / C$ is independent of $\left(\boldsymbol{W}^{-}, \boldsymbol{W}^{+}, \boldsymbol{X}, S, C, A\right)$. It may seem odd at first to choose $\ell_{i}=2 c_{i}$ (or any other multiple strictly larger than 1) instead of $\ell_{i}=c_{i}$ to express the default of institution $i$. The reason is that in the proof of Theorem 4.6 below we want to use Theorem 3.3 which only considers the limiting random vector $\left(\boldsymbol{W}^{-}, \boldsymbol{W}^{+}, \boldsymbol{X}, S, C, L, A\right)$. It would then be possible that $L=C$ in the limit $n \rightarrow \infty$ while $L_{n}<C_{n}$ almost surely for all $n \in \mathbb{N}$. This situation would not be distinguishable from $L_{n}=C_{n}$ for all $n \in \mathbb{N}$ and in order to derive meaningful results in Theorem 4.6 we have to choose $\ell_{i}>c_{i}$. Since $\stackrel{\circ}{\rho}_{\beta}(u)=\rho_{\beta}(u)=\rho_{\beta}(1)$ for all $u>1, \beta \in[T]$, this does not affect the contagion process.

In contrast to Definition 4.1 of resilience, we call a financial system non-resilient if any small shock causes a lower bounded linear damage by bankrupt institutions.

Definition 4.5 (Non-resilience) A financial system is said to be non-resilient if there exists $\Delta>0$ such that $n^{-1} \mathcal{S}_{n, L}>\Delta$ w.h.p. for any $L$ with the above listed properties.

The following theorem identifies lower bounds for the final default fraction and sold shares.

Theorem 4.6 If the initial shock L satisfies above properties and $h^{m}(\chi)$ is strictly increasing in $\chi^{m}$ for all $m \in[M]$, then for any $\epsilon>0$ w.h.p.

$$
n^{-1} \mathcal{S}_{n, L}>\stackrel{\circ}{g}\left(z^{*}, \chi^{*}\right)-\epsilon \text { and } \chi_{n, L}^{m}>\left(\chi^{*}\right)^{m}-\epsilon .
$$

The assumption on $h(\chi)$ is a rather mild one and is satisfied for all standard choices for price impact functions such as linear price or log-linear price impact.

Corollary 4.7 (Non-resilience criterion) If $h^{m}(\chi)$ is strictly increasing in $\chi^{m}$ for all $m \in[M]$ and $\stackrel{\circ}{g}\left(z^{*}, \chi^{*}\right)>0$, then the system is non-resilient.

For most practical purposes Corollaries 4.3 and 4.7 hence fully determine whether a financial system is resilient or non-resilient.

\section{Applications and simulations}

In this section, we provide two applications of our theory. Example 5.1 has a twofold purpose: it demonstrates the joint impact of default contagion and fire sales. The model parameters are 
chosen in such a way that the financial system would be resilient with respect to either one of them but non-resilient with respect to their combination. Further, we provide simulations for finite networks in this setting to confirm the applicability of our asymptotic results also for reasonably sized financial systems. In Example 5.2, we derive sufficient capital requirements for very general combined financial systems of default contagion and fire sales. This extends results from [19] for pure default contagion and from [18] for pure fire sales. From a regulator's viewpoint, capital requirements are the main tool to manage the risk of financial institutions. Traditionally, sufficient capital requirements are computed for each institution on a standalone basis by applying some (univariate) monetary risk measure like for example Value-atRisk or Expected Shortfall. However, this approach fails to capture systemic effects, and a recent line of research aims at rectifying this deficiency by determining system-wide capital requirements based on various types of systemic (multivariate) monetary risk measures.

Historically, the first approach to systemic risk measures was to apply a univariate (monetary) risk measure to some aggregated system-wide risk factor, see [11,26,29] for an axiomatic characterization of this family of systemic risk measures. Capital requirements for individual institutions must then be computed by some rule to allocate the total systemic risk capital. An alternative approach is to determine the total systemic risk by computing sufficient capital requirements on an institutional level before aggregating to a system-wide risk factor, see $[4,6,21]$. In this context an important but difficult question is whether from an institutional point of view the systemic capital requirements correspond to fair shares of the overall systemic risk, see e.g. [6]. In particular, one of the major problems in the implementation of the methodologies proposed in the literature mentioned above would be the fact that a given individual systemic capital requirement in general depends on the configuration of the complete system. As a consequence, one institution's capital requirement might be manipulated by other institutions' behaviour, or the entrance of a new institution into the system would potentially alter the capital requirements of all other institutions (even without direct business relations), for example.

One important contribution of the asymptotic methodology proposed in this paper is that, besides certain global parameters that need to be determined by a regulating institution, the implied systemic capital requirement for a given institution $i \in[n]$ only depends on its asset holdings $\boldsymbol{x}_{i}$ and its in-weights $w_{i}^{-, r, \alpha}$, which can be thought of as in-degrees, see [19]. They are thus very transparent and can be computed locally by the institutions themselves as they only depend on the institutions' own business decisions. This prevents institutions from manipulating their own or others' capital requirements and enables a fair allocation of systemic risk in the financial network. For simplicity we assume $S \equiv 1$ throughout this section and hence consider the final default fraction as the measure of systemic damage.

Example 5.1 Consider a financial system with $R=T=M=1$. For simplicity we omit superscripts throughout this example where appropriate. Let $w_{i}^{-}=w_{i}^{+}=x_{i}$ for each $i \in[n]$ and $W^{-}=W^{+}=X$ be Pareto distributed with density $f_{X}(x)=2 x^{-3} \mathbf{1}\{x \geq 1\}$. Further, let $c_{i}=3.5$ for each $i \in[n]$ and in particular $C=3.5$. Finally assume $h(\chi)=1-e^{-\chi}$ and $\rho(u)=\mathbf{1}\{u \geq 1\}$, that is banks sell assets at default only.

Since $c_{i}>3$, the system without fire sales would then be resilient (see [19, Theorem 3.7]). Also the pure fire sales system without loans would be resilient by [18, Corollary 1] since for $\chi \leq h^{-1}(3.5)$

$$
\begin{aligned}
f(\chi) & =\mathbb{E}[X \mathbf{1}\{X \geq 3.5 / h(\chi)\}]-\chi=\int_{3.5 / h(\chi)}^{\infty} 2 x^{-2} \mathrm{~d} x-\chi=\frac{4}{7} h(\chi)-\chi \\
& =\frac{4}{7}\left(1-e^{-\chi}\right)-\chi
\end{aligned}
$$




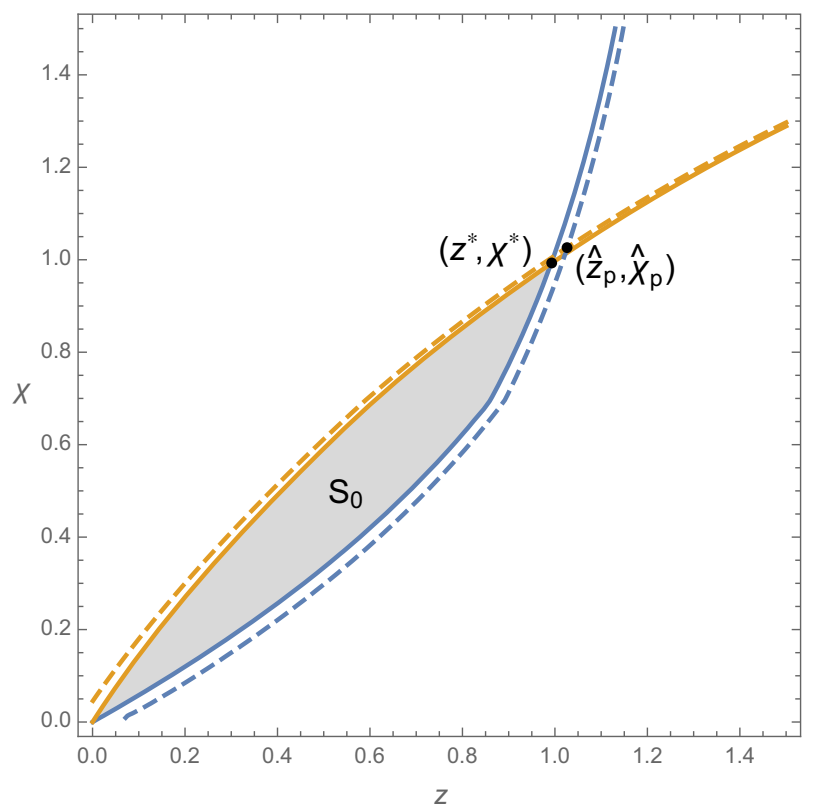

Fig. 2 Plot of the root sets of the functions $f^{1,1,1}(z, \chi)$ (blue) and $f^{1}(z, \chi)$ (orange). Solid: the unshocked functions. Dashed: the shocked functions. In grey the set $P=P_{0}$. (Color figure online)

and hence $f^{\prime}(0)=-3 / 7<0$. However, for the combined contagion system we derive that

$$
\begin{aligned}
f^{1,1,1}(z, \chi)= & +2 z\left(\operatorname{Ei}\left(-\frac{7 z}{2 h(\chi)}\right)-\operatorname{Ei}\left(-\frac{5 z}{2 h(\chi)}\right)\right)+\frac{4 h(\chi)}{7} e^{-\frac{7 z}{2 h(\chi)}}+z e^{-\frac{3 z}{2 h(\chi)}}-(z+2) e^{-z} \\
& -\frac{z}{3}\left(-e^{-\frac{z}{2 h(\chi)}}\left(\frac{z}{2 h(\chi)}+1\right)+e^{-z}(z+1)\right) \mathbf{1}\{\chi \leq \log 2\}-z, \\
f^{1}(z, \chi)= & f_{0}(z, \chi)+z-\chi
\end{aligned}
$$

where $\operatorname{Ei}(x):=\int_{-\infty}^{x} t^{-1} e^{t} \mathrm{~d} t$ denotes the exponential integral. In particular, $f^{1,1,1}(z, z)=$ $f^{1}(z, z)$ and

$$
\left.\frac{\mathrm{d}}{\mathrm{d} z} f^{1,1,1}(z, z)\right|_{z=0}=-\frac{1}{3}+\frac{1}{2} e^{-\frac{1}{2}}+e^{-\frac{3}{2}}+\frac{4}{7} e^{-\frac{7}{2}}+2\left(\operatorname{Ei}\left(-\frac{7}{2}\right)-\operatorname{Ei}\left(-\frac{5}{2}\right)\right) \approx 0.2462>0 .
$$

Hence the directional derivatives of $f^{1,1,1}$ and $f^{1}$ in direction $(1,1)$ are both positive and thus $z^{*}>0$ and $\chi^{*}>0$. See Fig. 2 for an illustration.

More precisely, we numerically determine $\left(z^{*}, \chi^{*}\right) \approx(0.992,0.992)$ and since $g(z, \chi)=$ $\stackrel{\circ}{g}(z, \chi)$ is given by

$$
\begin{aligned}
g(z, \chi)= & 1+z^{2}\left(2 \operatorname{Ei}\left(-\frac{5 z}{2 h(\chi)}\right)-\operatorname{Ei}\left(-\frac{3 z}{2 h(\chi)}\right)-\operatorname{Ei}\left(-\frac{7 z}{2 h(\chi)}\right)\right)-(z+1) e^{-z} \\
& +\frac{2 h(\chi)}{7}\left(\frac{2 h(\chi)}{7}-z\right) e^{-\frac{7 z}{2 h(\chi)}}+\frac{4}{5} z h(\chi) e^{-\frac{5 z}{2 h(\chi)}}+\frac{z^{2}}{3}\left(e^{-\frac{z}{2 h(\chi)}}-e^{-z}\right) \mathbf{1}\{\chi \leq \log 2\}
\end{aligned}
$$

a lower bound on the final default fraction is asymptotically given by $g\left(z^{*}, \chi^{*}\right) \approx 29.24 \%$. The combined system is thus non-resilient. 
Fig. 3 The simulation outcomes for systems as described in Example 5.1. In blue single outcomes, in orange the mean over all outcomes and in red the theoretical asymptotic final fraction. (Color figure online)
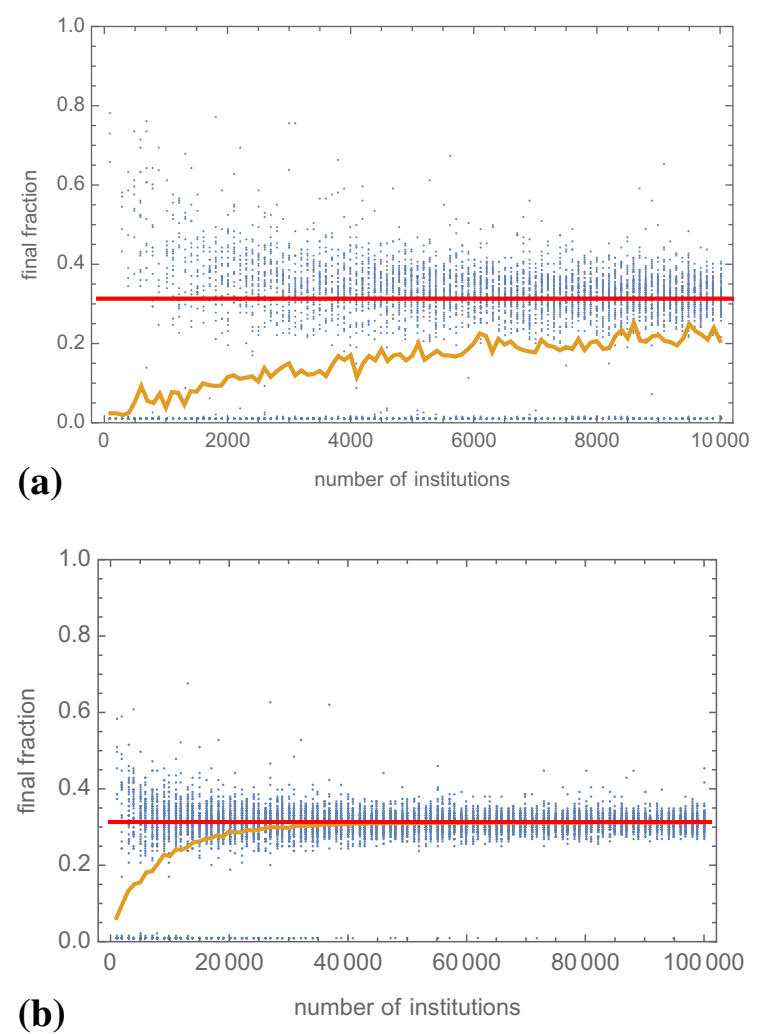

(b)

If we let each bank in the system initially default with probability $p=1 \%$, then we can determine $\left(\hat{z}_{p}, \hat{\chi}_{p}\right)=\left(z_{p}^{*}, \chi_{p}^{*}\right) \approx(1.028,1.028)$ as the unique joint root of the functions $f^{0}(z, \chi)=f_{p}^{1,1,1}(z, \chi)=(1-p) f^{1,1,1}(z, \chi)+p(2-z)$ and $\stackrel{\circ}{f}^{1}(z, \chi)=f_{p}^{1}(z, \chi)=$ $(1-p) f^{1}(z, \chi)+p(2-\chi)$. Plugging it into $\stackrel{\circ}{g}_{p}(z, \chi)=g_{p}(z, \chi)=(1-p) g(z, \chi)+p$ yields an asymptotic final fraction of $31.32 \%$.

To verify this result for finite systems, we performed $10^{5}$ simulations on systems of sizes between $10^{2}$ and $10^{4}(1000$ simulations for every multiple of 100$)$ as well as $10^{5}$ simulations on systems of sizes between $10^{3}$ and $10^{5}$ (1000 simulations for every multiple of 1000), where we drew $x_{i}$ randomly according to the limiting distribution of $X$. Figure 3 shows the mean over all 1000 simulations as an orange curve. Additionally, 100 simulations for every system size are depicted by blue dots. The theoretical final fraction of $31.32 \%$ is drawn as a red line. While for small $n$ only few simulations ended in a final default fraction significantly larger than $p=1 \%$ and those which did were considerably higher than the theoretical value of $31.32 \%$, as $n$ becomes larger, the average final fraction converges to $31.32 \%$ and the deviation around this value becomes smaller and smaller. Already for $n \approx 4,000$ the simulated and the theoretical results are considerably close.

Example 5.2 (Capital requirements) In the previous example we considered the case that $\rho(u)=\mathbf{1}\{u \geq 1\}$, i.e. sales at default only. Intermediate sales will make the system less resilient, and we shall consider such an example now. We choose $\rho(u)=1 \wedge u^{q}$ for some $q>0$. We consider one asset only and the parameter $q$ could be understood as a measure for 
the banks' confidence in the asset. Further, assume that the price impact is $h(\chi)=\Theta\left(\chi^{\nu}\right)$ for small $\chi$ and $v \geq q^{-1}$, i.e. there exist constants $\mu_{1}, \mu_{2} \in(0, \infty)$ such that $\mu_{1} \chi^{v} \leq h(\chi) \leq$ $\mu_{2} \chi^{\nu}$ for $\chi \leq \chi_{0}$ small enough. The generalization to multiple assets is straight forward in analogy to [18, Corollary 5].

The distribution of asset holdings is assumed to have a power law tail in the sense that $1-F_{X}(x)=\Theta\left(x^{1-\beta}\right)$ for some $\beta \in(2, \infty)$, i.e. there exist constants $B_{1}, B_{2} \in(0, \infty)$ such that $B_{1} x^{1-\beta} \leq 1-F_{X}(x) \leq B_{2} x^{1-\beta}$ for $x \geq x_{0}$ large enough.

First assume that $R=T=1$. Recall then from [19, Theorem 3.11] the sufficient (and necessary) capital requirements for a pure default contagion model without fire sales: Assume $1-F_{W^{ \pm}}(w) \leq\left(w / K^{ \pm}\right)^{1-\beta^{ \pm}}$for constants $K^{ \pm} \in(0, \infty)$ and $\beta^{ \pm}>2$, and for $w \geq w_{0} \in \mathbb{R}_{+}$. That is, the tails of the distributions of $W^{-}$and $W^{+}$are at most of power $\beta^{-}$resp. $\beta^{+}$. If we let $\gamma_{c}:=2+\frac{\beta^{-}-1}{\beta^{+}-1}-\beta^{-}$and $c_{i}=c\left(w_{i}^{-}\right)$for each bank $i \in[n]$ with $c: \mathbb{R}_{+, 0} \rightarrow$ $(1, \infty)$, then the (pure default contagion) system is resilient if either $\gamma_{c}<0, \gamma_{c}>0$ and $\liminf _{w \rightarrow \infty} w^{-\gamma_{c}} c(w)>\frac{\beta^{+}-1}{\beta^{+}-2} K^{+}\left(K^{-}\right)^{1-\gamma_{c}}=: \alpha_{c}$ or $\gamma_{c}=0$ and $\liminf _{w \rightarrow \infty} c(w)>$ $\alpha_{c}+1$. It thus makes sense to define capital requirements $c^{\operatorname{dir}}(w)=\max \left\{2, \alpha w^{\gamma}\right\}$ for some constants $\alpha>\alpha_{c}$ and $\gamma \geq \gamma_{c}$.

Adding the capital requirements $c^{\text {dir }}$ against direct contagion to the sufficient capital requirements $c^{\text {ind }}(x)=\theta x$ against fire sales (indirect contagion) found in [18, Corollary 4], we thus get the combined capital requirement $c_{i} \geq c\left(w_{i}^{-}, x_{i}\right)$ for each $i \in[n]$, where

$$
c(w, x)=\max \left\{2, \alpha w^{\gamma}\right\}+\theta x .
$$

In fact, we can show that these capital requirements make the combined system resilient: It holds

$$
f^{1}(0, \chi)=\mathbb{E}\left[X \min \left\{1,\left(\frac{X h(\chi)}{C}\right)^{q}\right\}\right]-\chi<0
$$

for $\chi>0$ small enough since $C \geq \theta X$. Since $f^{1}(z, \chi)$ is continuous in $z$ for fixed $\chi$, we can then choose $z>0$ small enough such that still $f^{1}(z, \chi)<0$. Furthermore, it holds for $\chi<h^{-1}(\theta)$ and $z>0$ small enough that

$$
f^{1,1,1}(z, \chi) \leq \mathbb{E}\left[W^{+} \mathbb{P}\left(\operatorname{Poi}\left(W^{-} z\right) \geq \max \left\{2, \alpha\left(W^{-}\right)^{\gamma}\right\}\right)\right]-z<0
$$

by resilience of the pure default contagion system (see the proof of [19, Theorem 3.11]). By definition of $\left(z^{*}, \chi^{*}\right)$ we can then conclude $z^{*}<z$ and $\chi^{*}<\chi$. However, $z$ and $\chi$ can be chosen arbitrarily small and thus $z^{*}=\chi^{*}=0$. The combined system is then resilient by Corollary 4.3 .

\section{Model estimation procedure}

Our model describes how distress propagates through financial systems by default contagion and fire sales. The previous section shows that our analytic results, although derived asymptotically for large systems, approximate cascades in finite systems well. When using our model to analyze the stability of some observed real financial network, however, it is clearly necessary to calibrate the used model parameters to observed quantities. For this reason, we shall discuss how such a calibration can be achieved.

We start with the type assignment. One of the core assumptions of our model is the classification of institutions into certain blocks by their type. While this assignment can be obvious with regard to certain institution characteristics such as its business model (retail 
bank, hedge fund, insurance company, etc.) or its geographical location, other traits can be more challenging to detect and require a more sophisticated calibration. In particular, we need to group institutions into communities and identify core-periphery structures to describe real financial networks in a proper way. The literature on such detection algorithms, however, is well developed. See for example [7,13,15,22,36,37], respectively [27,33].

Having assigned a certain institution type to all members of the financial system, the next challenge is the choice of vertex weight parameters for each of them. In the following, we propose an estimation of weights based on likelihood maximization. For this purpose, we assume that for each institution the distribution of its exposures $r=1 \ldots R$ towards institutions of a given type is known. Note that the (distribution of) exposures might not be directly observable and possibly need to be estimated.

In our model for any two types $\alpha, \beta \in[T]$ and $r \in[R]$ a subsystem of links is induced (all links of exposure size $r$ going from an $\alpha$-institution to a $\beta$-institution) and the calibration of $\left\{w_{i}^{+, r, \beta}, w_{j}^{-, r, \alpha}\right\}_{i, j \in[n]: \alpha_{i}=\alpha, \alpha_{j}=\beta}$ is independent from all other subsystems. To simplify the discussion in the following, we thus consider a one-type $(T=1)$ system with constant exposure of $1(R=1)$ and abbreviate $w_{i}^{ \pm, 1,1}$ by $w_{i}^{ \pm}$. By the same methods any other subsystem can be calibrated to observed data.

First note that for a network $G$ of size $n$ with edge set $E(G)$ the likelihood of weight sequences $\boldsymbol{w}^{-}=\left(w_{1}^{-}, \ldots, w_{n}^{-}\right)$and $\boldsymbol{w}^{+}=\left(w_{1}^{+}, \ldots, w_{n}^{+}\right)$is given by

$$
L\left(w_{1}^{-}, w_{1}^{+}, \ldots, w_{n}^{-}, w_{n}^{+} \mid E(G)\right)=\prod_{(i, j) \in E(G)}\left(\frac{w_{i}^{+} w_{j}^{-}}{n} \wedge 1\right) \prod_{\substack{(i, j) \notin E(G) \\ i \neq j}}\left(1-\frac{w_{i}^{+} w_{j}^{-}}{n} \wedge 1\right) .
$$

One can always derive the maximum-likelihood estimators $\hat{w}_{1}^{-}, \ldots, \hat{w}_{n}^{-}, \hat{w}_{1}^{+}, \ldots, \hat{w}_{n}^{+}$by numerically maximizing $L$. In order to obtain some intuition about them, we further want to derive an approximation of the estimators. For this, we assume that $w_{i}^{+} w_{j}^{-} \ll n$ for all $i, j \in[n]$ which is a reasonable assumption at least when $W^{+}, W^{-}$are square-integrable. We can hence approximate

$$
L\left(w_{1}^{-}, w_{1}^{+}, \ldots, w_{n}^{-}, w_{n}^{+} \mid E(G)\right) \approx \frac{1}{n^{s}} \prod_{i \in[n]}\left(w_{i}^{-}\right)^{d_{i}^{-}}\left(w_{i}^{+}\right)^{d_{i}^{+}} \exp \left(-w_{i}^{+} \frac{\sum_{j \in[n]} w_{j}^{-}}{n}\right),
$$

where $s:=\sum_{i \in[n]} d_{i}^{-}=\sum_{i \in[n]} d_{i}^{+}$, and $d_{i}^{-}$and $d_{i}^{+}$are the in and out degree of institution $i$, respectively. By the product form $w_{i}^{+} w_{j}^{-}$in (3.1), we are free to multiply all out-weights $w_{i}^{+}$by some constant $\eta$ if, at the same time, we multiply all in-weights by its inverse $\eta^{-1}$. Motivated by the fact that $\sum_{i \in[n]} d_{i}^{-}=\sum_{i \in[n]} d_{i}^{+}$, we use this degree of freedom to set $\sum_{i \in[n]} w_{i}^{-}=\sum_{i \in[n]} w_{i}^{+}$and want to maximize the approximated likelihood function under this constraint. (Other constraints, such as $\sum_{i \in[n]} w_{i}^{-}=$const, are also possible and lead to the same result in the end.) By Lagrange's multiplier method this leads to a maximization of

$$
\prod_{i \in[n]}\left(w_{i}^{-}\right)^{d_{i}^{-}}\left(w_{i}^{+}\right)^{d_{i}^{+}} \exp \left(-w_{i}^{+} \frac{\sum_{j \in[n]} w_{j}^{-}}{n}\right)+\lambda\left(\sum_{k \in[n]} w_{k}^{-}-w_{k}^{+}\right) \text {. }
$$

Differentiating with respect to $w_{l}^{-}$resp. $w_{l}^{+}$for all $l \in[n]$, we are left with solving the equations

$$
0=\prod_{i \in[n]}\left(w_{i}^{-}\right)^{d_{i}^{-}}\left(w_{i}^{+}\right)^{d_{i}^{+}} \exp \left(-w_{i}^{+} \frac{\sum_{j \in[n]} w_{j}^{-}}{n}\right)\left(\frac{d_{l}^{-}}{w_{l}^{-}}-\frac{\sum_{k \in[n]} w_{k}^{+}}{n}\right)+\lambda
$$


respectively

$$
0=\prod_{i \in[n]}\left(w_{i}^{-}\right)^{d_{i}^{-}}\left(w_{i}^{+}\right)^{d_{i}^{+}} \exp \left(-w_{i}^{+} \frac{\sum_{j \in[n]} w_{j}^{-}}{n}\right)\left(\frac{d_{l}^{+}}{w_{l}^{+}}-\frac{\sum_{k \in[n]} w_{k}^{-}}{n}\right)-\lambda .
$$

In particular, $d_{l}^{-} / w_{l}^{-}$resp. $d_{l}^{+} / w_{l}^{+}$must be independent of $l$ and we can thus find constants $\lambda^{-}$and $\lambda^{+}$such that $w_{l}^{-}=\lambda^{-} d_{l}^{-}$and $w_{l}^{+}=\lambda^{+} d_{l}^{+}$. Using the constraints $\sum_{i \in[n]} w_{i}^{-}=\sum_{i \in[n]} w_{i}^{+}$and $\sum_{i \in[n]} d_{i}^{-}=\sum_{i \in[n]} d_{i}^{+}$, we obtain that $\lambda=0$ and $\lambda^{-}=\lambda^{+}=\sqrt{n / \sum_{i \in[n]} d_{i}^{-}}$such that the approximated likelihood function is maximized by

$$
w_{i}^{-}=d_{i}^{-} \sqrt{\frac{n}{\sum_{j \in[n]} d_{j}^{-}}}, \quad w_{i}^{+}=d_{i}^{+} \sqrt{\frac{n}{\sum_{j \in[n]} d_{j}^{-}}} .
$$

That is, the approximated weight estimators are proportional to the observed degrees and only normalized in a certain sense.

The smaller the observed fraction $\max _{i, j \in[n]} d_{i}^{+} d_{j}^{-} / \sum_{k \in[n]} d_{k}^{-}$, the better is above approximation of $w_{i}^{+} w_{j}^{-}=d_{i}^{+} d_{j}^{-} n / \sum_{k \in[n]} d_{k}^{-} \ll n$. On networks where $\max _{i, j \in[n]} d_{i}^{+} d_{j}^{-} /$ $\sum_{k \in[n]} d_{k}^{-}$is large, $\boldsymbol{w}^{-}$and $\boldsymbol{w}^{+}$have to be estimated numerically. After each institution has been assigned a type $\alpha_{i}$ according to one of the above cited methods and their weights $w_{i}^{-}$ and $\boldsymbol{w}_{i}^{+}$have been estimated, it remains to fit a multivariate distribution to the empirical distribution of $\left\{\left(\boldsymbol{w}_{i}^{-}, \boldsymbol{w}_{i}^{+}, c_{i}, \alpha_{i}\right)\right\}_{i \in[n]}$ in order to completely determine the distribution of $\left(\boldsymbol{W}^{-}, \boldsymbol{W}^{+}, C, A\right)$.

\section{Discussion}

In this paper we propose an asymptotic model to analyze contagion in financial networks. It combines two important channels of contagion: default contagion and fire sales. It can be specified by the joint distribution of capital, asset holdings, network liabilities and other relevant quantities. There is no restriction on the joint distribution of the parameters except that we require existence of a first moment. This makes the model rather general and, thanks to its asymptotic nature, allows the parameters of the model to be chosen from all kinds of distribution as Pareto, Exponential, Gamma or Fréchet to name just a few. The sales functions $\rho_{\alpha}, \alpha \in[T]$ allow to specify (and test accordingly) different regulatory environments. For example in a crisis, a regulator could use the model as a decision guidance to decide whether to suspend the enforcement of certain leverage requirements in order to stop a contagion spiral driven by asset sales, aimed to fulfil them. See [12] for a discussion of events that triggered such regulatory actions in the past. Moreover, the model serves as a decision tool for the search of the most favourable financial systems and regulatory environments. The results provided in Theorems 3.3, 4.2 and 4.6 allow to test different stress scenarios easily without complicated and computationally heavy simulation of the entire contagion process. Only the functionals $g, f^{m}$ and $f^{r, \alpha, \beta}$ have to be calculated, which (if an analytic expression is not available for the chosen parameters) can always be done via simple Monte Carlo simulation. As we have seen in Examples 5.1 and 5.2, in some situations conclusions about resilience can even be derived without any simulation, on a purely analytic level.

Despite the generality of the model, results have to be taken with some caution. While it is a first step towards an integrated model within the asymptotic framework, it is not a 
fully fledged systemic risk analysis aiming to provide precise numerical predictions about the contagion outcome triggered by certain stress scenarios. Still, we believe that it serves as valuable tool for comparison of different regulatory measures and for identifying preferable structures of financial systems. Some of the most crucial simplifications of our model are:

- Conservative: The model is conservative in several regards: The recovery rate of defaulted institutions is assumed to be zero. This can be considered a reasonable assumption in the short term, considering that the resolution of defaulted institutions takes time. For example, the resolution of Lehman Brothers took over 10 years, and in a bond auction for the settlement of credit default swaps written on Lehman Brothers just three weeks after its default the realized recovery rate only amounted to $8.625 \%$ [30]. We remark, however, that all results in our model can be derived analogously for any other exogenously given (but possibly institution-dependent) recovery rate. Similarly, the fire-sales process is conservative as $\boldsymbol{x}_{i} \cdot h(\chi)$ serves only as an upper bound to the loss experienced by institution $i$ after $\chi$ shares have been sold.

- Asset purchase The model does not feature buyers who might jump in to profit from buying assets that have devalued due to the fire-sale spiral. These buyers are most likely part of the non-banking sector and serve as crucial liquidity providers as shown in [10]. While our block model allows to include these institutions even if they do not participate in the interbank lending market and are not selling assets, their positive effect as a liquidity provider can only be incorporated via the specification of the function $h$. Modelling asset purchases in an endogenous way, however, is not possible within our current model.

- Missing channels The model ignores other important channels of contagion as for example cross-holdings, liquidity contagion or bank runs.

- Static: While the model considers different rounds of contagion, it is static in the sense that model parameters are not changing over time. Due to the asymptotic nature variations on a local level are not likely to change the outcome as long as global characteristics are not changing. However, in a crisis, the entire system might change and adapt to the new environment.

- Systemically important financial institutions Assumption 3.1 of a regular vertex sequence ensures that as $n \rightarrow \infty$ the number of vertices with certain parameters stabilizes. This together with the first moment assumption in 3.1 and the specification of link probabilities (3.1) has the consequence that the influence of a finite set of institutions vanishes as $n \rightarrow \infty$. This might seem at odds with the idea that a small set of banks is systemically important and might have to be regulated differently. In our framework however, it is more natural to think of a small fraction of banks (of size $\varepsilon n$ ) to be systemically important. Within our block model framework one could then assign to them their own block $\alpha_{\text {SIFI }}$ and ensure that $\left(\boldsymbol{W}^{-}, \boldsymbol{W}^{+}\right)$is specified such that Systemically Important Financial Institutions build significantly more links than other institutions.

Some of the simplifications mentioned give rise to future investigations. Also, this paper focuses on the theoretical foundations, accompanied by a simulation study to show the accuracy of the asymptotic results for finite networks. While we study the implications of our model for few stylized examples, it is beyond the scope of the current paper and left for future research to apply this model to the many different possible settings. In particular the relatively advanced block model setup for the liability network allows to analyze many interesting shock distributions. To name just a few examples, one could analyze shock distributions targeting institutions of certain size only, institutions under certain regulatory requirements, institutions with large asset holdings or only institutions participating in the interbank market. 
Acknowledgements Open Access funding provided by Projekt DEAL.

Open Access This article is licensed under a Creative Commons Attribution 4.0 International License, which permits use, sharing, adaptation, distribution and reproduction in any medium or format, as long as you give appropriate credit to the original author(s) and the source, provide a link to the Creative Commons licence, and indicate if changes were made. The images or other third party material in this article are included in the article's Creative Commons licence, unless indicated otherwise in a credit line to the material. If material is not included in the article's Creative Commons licence and your intended use is not permitted by statutory regulation or exceeds the permitted use, you will need to obtain permission directly from the copyright holder. To view a copy of this licence, visit http://creativecommons.org/licenses/by/4.0/.

\section{Appendix}

\subsection{Proofs for Sect. 2}

Proof of Lemma 2.1 Existence of a smallest solution $\left(\overline{\mathcal{D}}_{n}, \bar{\chi}_{n}\right)$ follows from the KnasterTarski theorem.

Clearly, $\varnothing=\mathcal{D}_{(0)} \subseteq \overline{\mathcal{D}}_{n}$ and $\mathbf{0}=\sigma_{(0)} \leq \bar{\chi}_{n}$. So let us inductively assume that $\mathcal{D}_{(k)} \subseteq \overline{\mathcal{D}}_{n}$ and $n^{-1} \sigma_{(k)} \leq \bar{\chi}_{n}$ for some $0<k \in \mathbb{N}$. Then

$$
\begin{aligned}
\mathcal{D}_{(k+1)} & =\left\{i \in[n]: \sum_{j \in \mathcal{D}_{(k)}} e_{j, i} \geq c_{i}-\ell_{i}-\boldsymbol{x}_{i} \cdot h\left(n^{-1} \boldsymbol{\sigma}_{(k)}\right)\right\} \\
& \subseteq\left\{i \in[n]: \sum_{j \in \overline{\mathcal{D}}_{n}} e_{j, i} \geq c_{i}-\ell_{i}-\boldsymbol{x}_{i} \cdot h\left(\bar{\chi}_{n}\right)\right\}=\overline{\mathcal{D}}_{n}
\end{aligned}
$$

and

$$
\begin{aligned}
n^{-1} \boldsymbol{\sigma}_{(k+1)} & =n^{-1} \sum_{i \in[n]} \boldsymbol{x}_{i} \odot \rho_{i}\left(\frac{\sum_{j \in \mathcal{D}_{(k)}} e_{j, i}+\ell_{i}+\boldsymbol{x}_{i} \cdot h\left(n^{-1} \boldsymbol{\sigma}_{(k)}\right)}{c_{i}}\right) \\
& \leq n^{-1} \sum_{i \in[n]} \boldsymbol{x}_{i} \odot \rho_{i}\left(\frac{\sum_{j \in \overline{\mathcal{D}}_{n}} e_{j, i}+\ell_{i}+\boldsymbol{x}_{i} \cdot h\left(\overline{\boldsymbol{\chi}}_{n}\right)}{c_{i}}\right)=\bar{\chi}_{n} .
\end{aligned}
$$

In particular, this shows that $\mathcal{D}_{n}=\bigcup_{k \in \mathbb{N}} \mathcal{D}_{(k)} \subseteq \overline{\mathcal{D}}_{n}$ and $\chi_{n}=n^{-1} \lim _{k \rightarrow \infty} \sigma_{(k)} \leq \bar{\chi}_{n}$.

Proof of Lemma 2.2 Existence of a smallest solution $\left(\hat{\mathcal{D}}_{n}, \hat{\chi}_{n}\right)$ follows from the KnasterTarski theorem. Consider now the adjusted contagion process $\tilde{\mathcal{D}}_{(0)}=\mathcal{D}_{(0)}=\emptyset, \tilde{\boldsymbol{\sigma}}_{(0)}=$ $\boldsymbol{\sigma}_{(0)}=\mathbf{0}$ and

$$
\tilde{\mathcal{D}}_{(k)}=\left\{i \in[n]: \sum_{j \in \tilde{\mathcal{D}}_{(k-1)}} e_{j, i}>c_{i}-\ell_{i}-\boldsymbol{x}_{i} \cdot h\left(n^{-1} \tilde{\boldsymbol{\sigma}}_{(k-1)}\right)\right\}
$$

respectively

$$
\tilde{\boldsymbol{\sigma}}_{(k)}=\sum_{i \in[n]} \boldsymbol{x}_{i} \odot \stackrel{\circ}{\rho}_{i}\left(\frac{\sum_{j \in \tilde{\mathcal{D}}_{(k-1)}} e_{j, i}+\ell_{i}+\boldsymbol{x}_{i} \cdot h\left(n^{-1} \tilde{\boldsymbol{\sigma}}_{(k-1)}\right)}{c_{i}}\right),
$$

for $k \geq 1$. This process converges and clearly it holds

$$
\tilde{\mathcal{D}}_{n}:=\bigcup_{k \in \mathbb{N}} \tilde{\mathcal{D}}_{(k)} \subseteq \bigcup_{k \in \mathbb{N}} \mathcal{D}_{(k)}=\mathcal{D}_{n} \quad \text { and } \quad \tilde{\chi}_{n}:=n^{-1} \lim _{k \rightarrow \infty} \tilde{\boldsymbol{\sigma}}_{(k)} \leq n^{-1} \lim _{k \rightarrow \infty} \boldsymbol{\sigma}_{(k)}=\chi_{n} .
$$


We will now show that $\left(\tilde{\mathcal{D}}_{n}, \tilde{\chi}_{n}\right)$ is a solution to (2.5) and (2.6) such that in particular $\hat{\mathcal{D}}_{n} \subseteq \tilde{\mathcal{D}}_{n} \mathcal{D}_{n}$ and $\hat{\chi}_{n} \leq \tilde{\chi}_{n} \leq \chi_{n}$. Clearly,

$$
\begin{aligned}
\tilde{\mathcal{D}}_{(k)} & =\left\{i \in[n]: \sum_{j \in \tilde{\mathcal{D}}_{(k-1)}} e_{j, i}>c_{i}-\ell_{i}-\boldsymbol{x}_{i} \cdot h\left(n^{-1} \tilde{\boldsymbol{\sigma}}_{(k-1)}\right)\right\} \\
& \subseteq\left\{i \in[n]: \sum_{j \in \tilde{\mathcal{D}}_{n}} e_{j, i}>c_{i}-\ell_{i}-\boldsymbol{x}_{i} \cdot h\left(\tilde{\boldsymbol{\chi}}_{n}\right)\right\}
\end{aligned}
$$

and thus

$$
\tilde{\mathcal{D}}_{n}=\bigcup_{k \in \mathbb{N}} \tilde{\mathcal{D}}_{(k)} \subseteq\left\{i \in[n]: \sum_{j \in \tilde{\mathcal{D}}_{n}} e_{j, i}>c_{i}-\ell_{i}-\boldsymbol{x}_{i} \cdot h\left(\tilde{\chi}_{n}\right)\right\} .
$$

On the other hand, if for $i \in[n]$ it holds

$$
\sum_{j \in \tilde{\mathcal{D}}_{n}} e_{j, i}>c_{i}-\ell_{i}-\boldsymbol{x}_{i} \cdot h\left(\tilde{\chi}_{n}\right),
$$

then by continuity of $h$ we find $k \in \mathbb{N}$ large enough such that

$$
\sum_{j \in \tilde{\mathcal{D}}_{n}} e_{j, i}>c_{i}-\ell_{i}-\boldsymbol{x}_{i} \cdot h\left(n^{-1} \tilde{\boldsymbol{\sigma}}_{(k)}\right) .
$$

Without loss of generality assume that $k$ is large enough such that $\tilde{\mathcal{D}}_{(k)}=\tilde{\mathcal{D}}_{n}$. Then in particular $i \in \tilde{\mathcal{D}}_{(k)}=\tilde{\mathcal{D}}_{n}$ and

$$
\tilde{\mathcal{D}}_{n}=\left\{i \in[n]: \sum_{j \in \tilde{\mathcal{D}}_{n}} e_{j, i}>c_{i}-\ell_{i}-\boldsymbol{x}_{i} \cdot h\left(\tilde{\chi}_{n}\right)\right\} .
$$

That is, $\tilde{\mathcal{D}}_{n}$ and $\tilde{\chi}_{n}$ solve (2.5). Moreover, they solve (2.6) as

$$
\begin{aligned}
\tilde{\boldsymbol{\chi}}_{n} & =n^{-1} \lim _{k \rightarrow \infty} \tilde{\boldsymbol{\sigma}}_{(k)}=n^{-1} \lim _{k \rightarrow \infty} \sum_{i \in[n]} \boldsymbol{x}_{i} \odot \stackrel{\circ}{\rho}_{i}\left(\frac{\sum_{j \in \tilde{\mathcal{D}}_{(k-1)}} e_{j, i}+\ell_{i}+\boldsymbol{x}_{i} \cdot h\left(n^{-1} \tilde{\boldsymbol{\sigma}}_{(k-1)}\right)}{c_{i}}\right) \\
& =n^{-1} \sum_{i \in[n]} \boldsymbol{x}_{i} \odot \stackrel{\circ}{\rho}_{i}\left(\frac{\sum_{j \in \tilde{\mathcal{D}}_{n}} e_{j, i}+\ell_{i}+\boldsymbol{x}_{i} \cdot h\left(\tilde{\boldsymbol{\chi}}_{n}\right)}{c_{i}}\right)
\end{aligned}
$$

where we used left continuity of $h, \rho_{\rho}, i \in[n]$, and the fact that $\tilde{\mathcal{D}}_{(k)}=\tilde{\mathcal{D}}_{n}$ for $k$ large enough.

\subsection{Proofs for Sect. 3}

We first consider the special case, summarized in the following definition, where the weights, asset holdings, systemic importance levels, capitals and exogenous losses take only finitely many different values.

Definition 8.1 (Finitary regular vertex sequence) A regular vertex sequence (see Assumption 3.1) denoted by $\left(\boldsymbol{w}^{-}(n), \boldsymbol{w}^{+}(n), \boldsymbol{x}(n), \boldsymbol{s}(n), \boldsymbol{c}(n), \boldsymbol{\ell}(n), \boldsymbol{\alpha}(n)\right)_{n \in \mathbb{N}}$ is called finitary if there exist $J \in \mathbb{N}$ and a finite set $\left\{\left(\tilde{\boldsymbol{w}}_{j}^{-}, \tilde{\boldsymbol{w}}_{j}^{+}, \tilde{\boldsymbol{x}}_{j}, \tilde{s}_{j}, \tilde{c}_{j}, \tilde{\ell}_{j}\right)\right\}_{j \in[J]} \subset \mathbb{R}_{+, 0}^{[R] \times[T]} \times \mathbb{R}_{+, 0}^{[R] \times[T]} \times$ 


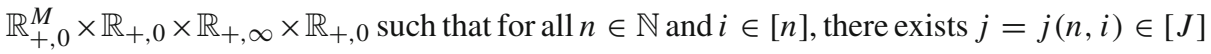
such that $\left(\boldsymbol{w}_{i}^{-}, \boldsymbol{w}_{i}^{+}, \boldsymbol{x}_{i}, s_{i}, c_{i}, \ell_{i}\right)=\left(\tilde{\boldsymbol{w}}_{j}^{-}, \tilde{\boldsymbol{w}}_{j}^{+}, \tilde{\boldsymbol{x}}_{j}, \tilde{s}_{j}, \tilde{c}_{j}, \tilde{\ell}_{j}\right)$. Denote in the following

$$
p_{j}^{\beta}(n):=\mathbb{P}\left(\boldsymbol{W}_{n}^{-}=\tilde{\boldsymbol{w}}_{j}^{-}, \boldsymbol{W}_{n}^{+}=\tilde{\boldsymbol{w}}_{j}^{+}, \boldsymbol{X}_{n}=\tilde{\boldsymbol{x}}_{j}, S_{n}=\tilde{s}_{j}, C_{n}=\tilde{c}_{j}, L_{n}=\tilde{\ell}_{j}, A_{n}=\beta\right)
$$

and

$p_{j}^{\beta}=\lim _{n \rightarrow \infty} p_{j}^{\beta}(n)=\mathbb{P}\left(\boldsymbol{W}^{-}=\tilde{\boldsymbol{w}}_{j}^{-}, \boldsymbol{W}^{+}=\tilde{\boldsymbol{w}}_{j}^{+}, \boldsymbol{X}=\tilde{\boldsymbol{x}}_{j}, S=\tilde{\boldsymbol{s}}_{j}, C=\tilde{c}_{j}, L=\tilde{\ell}_{j}, A=\beta\right)$.

We can then prove a version of Theorem 3.3 for the finitary case:

Theorem 8.2 Consider a financial system described by a finitary regular vertex sequence. Then for the final systemic damage $n^{-1} \mathcal{S}_{n}$ and $\chi_{n}^{m}$, the number of finally sold shares of asset $m \in[M]$ divided by $n$, it holds

$$
\begin{aligned}
\stackrel{\circ}{g}(\hat{z}, \hat{\chi})+o_{p}(1) & \leq n^{-1} \mathcal{S}_{n} \leq g\left(z^{*}, \chi^{*}\right)+o_{p}(1), \\
\hat{\chi}^{m}+o_{p}(1) & \leq \chi_{n}^{m} \leq\left(\chi^{*}\right)^{m}+o_{p}(1) .
\end{aligned}
$$

In particular, for the final price impact $h^{m}\left(\chi_{n}\right)$ on asset $m \in[M]$ it holds

$$
h^{m}(\hat{\chi})+o_{p}(1) \leq h^{m}\left(\chi_{n}\right) \leq h^{m}\left(\chi^{*}\right)+o_{p}(1) .
$$

The difficulty for this problem lies in the fact that the functions $f^{r, \alpha, \beta}$ are discontinuous in $\chi$. That is, there exist values for $\chi$ (sold assets) at which a linear fraction of banks defaults. However, $f^{r, \alpha, \beta}$ is discontinuous at $(\boldsymbol{z}, \chi)$ only if $\left(\tilde{c}_{j}-\tilde{\ell}_{j}-\tilde{\boldsymbol{x}}_{j} \cdot h(\chi)\right) \in \mathbb{N}$ for some $j \in J$ and there are hence only finitely many (possibly degenerated) hyperplanes of discontinuities.

Proof. We start with the proof of the lower bounds. That is, for arbitrary $\epsilon>0$ we will show that $n^{-1} \mathcal{S}_{n} \geq n^{-1} \hat{\mathcal{S}}_{n} \geq(1-\epsilon) \stackrel{\circ}{g}(\hat{z}, \hat{\chi})$ and $\chi_{n}^{m} \geq \hat{\chi}_{n}^{m} \geq(1-\epsilon) \hat{\chi}^{m}$ w.h.p. We therefore consider the contagion process given by rounds (i') and (ii'). That is, we first consider a cascade of default contagion. Once this cascade has ended (after at most $n-1$ steps) we start a cascade of fire sales and so on.

In order to quantify the default contagion cascade we use [31, Theorem 3.2.4], which extends the setting in [17] for systemic importance. That is, if we denote by $\hat{z}_{1} \in \mathbb{R}_{+, 0}^{V}$ the smallest vector such that

$$
\mathbb{E}\left[W^{+, r, \alpha} \mathbb{P}\left(\sum_{s \in[R]} s \text { Poi }\left(\sum_{\gamma \in[T]} W^{-, s, \gamma} \hat{z}_{1}^{s, \beta, \gamma}\right)>C-L\right) \mathbf{1}\{A=\beta\}\right]=\hat{z}_{1}^{r, \alpha, \beta}
$$

for all $(r, \alpha, \beta) \in V$, then the systemic importance of finally defaulted banks is bounded by

$$
(1-\delta) n \sum_{\beta \in[T]} \mathbb{E}\left[S \mathbb{P}\left(\sum_{s \in[R]} s \text { Poi }\left(\sum_{\gamma \in[T]} W^{-, s, \gamma} \hat{z}_{1}^{s, \beta, \gamma}\right)>C-L\right) \mathbf{1}\{A=\beta\}\right]
$$

from below w.h.p. for any fixed $\delta>0$. In fact, by finitariness of the system we can find $\theta>0$ small enough such that

$$
\begin{aligned}
\mathbb{E} & {\left[W^{+, r, \alpha} \mathbb{P}\left(\sum_{s \in[R]} s \text { Poi }\left(\sum_{\gamma \in[T]} W^{-, s, \gamma} z^{s, \beta, \gamma}\right)>C-L\right) \mathbf{1}\{A=\beta\}\right] } \\
& =\mathbb{E}\left[W^{+, r, \alpha} \mathbb{P}\left(\sum_{s \in[R]} s \operatorname{Poi}\left(\sum_{\gamma \in[T]} W^{-, s, \gamma} z^{s, \beta, \gamma}\right) \geq\lceil C-L+\theta\rceil\right) \mathbf{1}\{A=\beta\}\right]
\end{aligned}
$$


(note that $\lceil C-L+\theta\rceil$ is the weak limit of $\left\lceil C_{n}-L_{n}+\theta\right\rceil$ again by finitariness) and we are thus in the setting of [17]. However, while [31, Theorem 3.2.4] focuses on the systemic damage due to defaulted banks only, here it is also important to keep track of all losses due to defaults. In fact, the proof of [31, Theorem 3.2.4] for finitary systems shows that the number $q_{j, k}^{\beta}$ of institutions of class $j$ and type $\beta$ with a total edge weight from finally defaulted neighbors of at least $k \leq \tilde{c}_{j}$ is lower bounded by

$$
(1-\delta) n p_{j}^{\beta} \mathbb{P}\left(\sum_{s \in[R]} s \text { Poi }\left(\sum_{\gamma \in[T]} \tilde{w}_{j}^{-, s, \gamma} \hat{z}_{1}^{s, \beta, \gamma}\right) \geq k\right)
$$

w.h.p. for $\delta>0$. Since in this part of the proof we are interested in lower bounds, we assume in the following that $q_{j,\left\lceil\tilde{c}_{j}-\tilde{\ell}_{j}\right\rceil}^{\beta}=(1-\delta) n p_{j}^{\beta} \mathbb{P}\left(\sum_{s \in[R]} s \operatorname{Poi}\left(\sum_{\gamma \in[T]} \tilde{w}_{j}^{-, s, \gamma} \hat{z}_{1}^{s, \beta, \gamma}\right) \geq\left\lceil\tilde{c}_{j}-\right.\right.$ $\left.\left.\tilde{\ell}_{j}\right\rceil\right), q_{j, k}^{\beta}=(1-\delta) n p_{j}^{\beta} \mathbb{P}\left(\sum_{s \in[R]} s \operatorname{Poi}\left(\sum_{\gamma \in[T]} \tilde{w}_{j}^{-, s, \gamma} \hat{z}_{1}^{s, \beta, \gamma}\right)=k\right)$ for $1 \leq k<\left\lceil\tilde{c}_{j}-\tilde{\ell}_{j}\right\rceil$, and $q_{j, 0}^{\beta}=n p_{j}^{\beta}(n)-\sum_{k=1}^{\left\lceil\tilde{c}_{j}-\tilde{\ell}_{j}\right\rceil} q_{j, k}^{\beta}$ w.h.p. That is, we increase the losses due to default contagion.

Next, we want to use [18, Theorem EC. 1] to quantify the impact of the round of fire sales. We need to consider losses (and defaults in particular) due to the previous default contagion cascade. That is, we need to add to the exogenous losses $\ell_{i}$ the edge weight from defaulted debtors of each bank $i \in[n]$. This leads to a new loss vector $\left(\ell_{i}^{\prime}\right)_{i \in[n]}$. Note that we can set $\ell_{i}^{\prime}=\tilde{\ell}_{j}+\left\lceil\tilde{c}_{j}-\tilde{\ell}_{j}\right\rceil$ if $i$ is of type $j$ and the total edge-weight $k$ from finally defaulted debtors of $i$ is larger or equal to $\left\lceil\tilde{c}_{j}-\tilde{\ell}_{j}\right\rceil$. Denoting by $L_{n}^{\prime}$ a random vector distributed according to the empirical distribution function of $\left(\ell_{i}^{\prime}\right)_{i \in[n]}$, we thus derive that w.h.p.

$$
\begin{aligned}
& \mathbb{P}\left(\boldsymbol{W}_{n}^{-}=\tilde{\boldsymbol{w}}_{j}^{-}, \boldsymbol{W}_{n}^{+}=\tilde{\boldsymbol{w}}_{j}^{+}, \boldsymbol{X}_{n}=\tilde{\boldsymbol{x}}_{j}, S_{n}=\tilde{s}_{j}, C_{n}=\tilde{c}_{j}, L_{n}^{\prime}=\tilde{\ell}_{j}+k, A_{n}=\beta\right) \\
& \quad= \begin{cases}(1-\delta) p_{j}^{\beta} \mathbb{P}\left(\sum_{s \in[R]} s \operatorname{Poi}\left(\sum_{\gamma \in[T]} \tilde{w}_{j}^{-, s, \gamma} \hat{z}_{1}^{s, \beta, \gamma}\right) \geq\left\lceil\tilde{c}_{j}-\tilde{\ell}_{j}\right\rceil\right), & \text { if } k=\left\lceil\tilde{c}_{j}-\tilde{\ell}_{j}\right\rceil, \\
(1-\delta) p_{j}^{\beta} \mathbb{P}\left(\sum_{s \in[R]} s \operatorname{Poi}\left(\sum_{\gamma \in[T]} \tilde{w}_{j}^{-, s, \gamma} \hat{z}_{1}^{s, \beta, \gamma}\right)=k\right), & \text { if } 1 \leq k \leq\left\lceil\tilde{c}_{j}-\tilde{\ell}_{j}\right\rceil-1, \\
p_{j}^{\beta}(n)-(1-\delta) p_{j}^{\beta} \mathbb{P}\left(\sum_{s \in[R]} s \operatorname{Poi}\left(\sum_{\gamma \in[T]} \tilde{w}_{j}^{-, s, \gamma} \hat{z}_{1}^{s, \beta, \gamma}\right) \geq 1\right), & \text { if } k=0 .\end{cases}
\end{aligned}
$$

For simplicity in the notation, we assume that from $\left(\tilde{\boldsymbol{x}}_{j}, \tilde{s}_{j}, \tilde{c}_{j}, \tilde{\ell}_{j}\right)=\left(\tilde{\boldsymbol{x}}_{k}, \tilde{s}_{k}, \tilde{c}_{k}, \tilde{\ell}_{k}\right)$ it follows $j=k$ (i.e. classes $j$ and $k$ are not distinguished by their in- and out-weights only) in the following. Otherwise consider sums over classes with the same asset holdings, systemic importance, capital and exogenous loss.

In particular, for the weak limit $\left(\boldsymbol{X}, S, C, L^{\prime}, A\right)$ of $\left(\boldsymbol{X}_{n}, S_{n}, C_{n}, L_{n}^{\prime}, A_{n}\right)$ and $0 \leq k \leq$ $\left\lceil\tilde{c}_{j}-\tilde{\ell}_{j}\right\rceil$

$$
\begin{aligned}
& \mathbb{P}\left(\boldsymbol{X}=\tilde{\boldsymbol{x}}_{j}, S=\tilde{s}_{j}, C=\tilde{c}_{j}, L^{\prime}=\tilde{\ell}_{j}+k, A=\beta\right) \\
& \quad \geq(1-\delta) p_{j}^{\beta} \mathbb{P}\left(\sum_{s \in[R]} s \operatorname{Poi}\left(\sum_{\gamma \in[T]} \tilde{w}_{j}^{-, s, \gamma} \hat{z}_{1}^{s, \beta, \gamma}\right)=k\right) .
\end{aligned}
$$

Let now

$$
\stackrel{\circ}{f}_{\delta}^{m}(\chi):=\sum_{\beta \in[T]} \mathbb{E}\left[X^{m} \stackrel{\circ}{\rho}_{\alpha}^{m}\left(\frac{L^{\prime}+\boldsymbol{X} \cdot h(\chi)}{C}\right) \mathbf{1}\{A=\beta\}\right]-\chi^{m}, \quad m \in[M]
$$


the corresponding functions as in [18] adapted to the setting with heterogeneous sales functions and $\hat{\chi}_{\delta}$ its smallest fixed point. Then

$$
\begin{aligned}
f_{\delta}^{m} & (\chi)+\chi^{m} \\
= & \sum_{\beta \in[T]} \sum_{j \in[J]} \sum_{k \geq 0} \tilde{x}_{j}^{m} \stackrel{\circ}{\rho}_{\beta}^{m}\left(\frac{\tilde{\ell}_{j}+k+\tilde{\boldsymbol{x}}_{j} \cdot h(\chi)}{\tilde{c}_{j}}\right) \\
& \mathbb{P}\left(\boldsymbol{X}=\tilde{\boldsymbol{x}}_{j}, S=\tilde{s}_{j}, C=\tilde{c}_{j}, L^{\prime}=\tilde{\ell}_{j}+k, A=\beta\right) \\
\geq & (1-\delta) \sum_{\beta \in[T]} \sum_{j \in[J]} \sum_{k \geq 0} \tilde{x}_{j}^{m} \stackrel{\circ}{\rho}_{\beta}^{m}\left(\frac{\tilde{\ell}_{j}+k+\tilde{\boldsymbol{x}}_{j} \cdot h(\chi)}{\tilde{c}_{j}}\right) \\
& p_{j}^{\beta} \mathbb{P}\left(\sum_{s \in[R]} s \operatorname{Poi}\left(\sum_{\gamma \in[T]} \tilde{w}_{j}^{-, s, \gamma} \hat{z}_{1}^{s, \beta, \gamma}\right)=k\right) \\
= & (1-\delta)\left(\tilde{f}^{m}\left(\hat{\boldsymbol{z}}_{1}, \chi\right)+\chi^{m}\right)
\end{aligned}
$$

and $\stackrel{\circ}{f}_{\delta}^{m}(\chi) \geq(1-\delta) \stackrel{\circ}{f}^{m}\left(\hat{z}_{1}, \chi\right)-\delta \chi^{m} \geq \stackrel{\circ}{f}^{m}\left(\hat{z}_{1}, \chi\right)-\delta \mathbb{E}\left[X^{m}\right]$. In particular, $\lim \inf _{\delta \rightarrow 0+} \stackrel{\circ}{\delta}_{\delta}^{m}(\chi) \geq \stackrel{\circ}{f}^{m}\left(\hat{z}_{1}, \chi\right)$ and by [18, Lemma EC. 2] we derive that $\lim _{\inf } \rightarrow 0+\hat{\chi}_{\delta} \geq$ $\hat{\chi}_{1}$, where $\hat{\chi}_{1}$ denotes the smallest joint root of the functions $f^{m}\left(\hat{z}_{1}, \chi\right), m \in[M]_{0}$, for fixed $z=\hat{z}_{1}$.

We can hence choose $\delta$ small enough such that the number of finally sold shares of asset $m$ is lower bounded by $n(1-\epsilon) \hat{\chi}_{1}^{m}$ w.h.p. by [18, Theorem EC. 1]. Further, for

$$
\begin{aligned}
\stackrel{\circ}{g}_{\delta}(\chi) & :=\mathbb{E}\left[S \mathbf{1}\left\{L^{\prime}+\boldsymbol{X} \cdot h(\chi)>C\right\}\right] \\
= & \sum_{\beta \in[T]} \sum_{j \in[J]} \tilde{s}_{j} \sum_{k \geq 0} \mathbf{1}\left\{k>\tilde{c}_{j}-\tilde{\ell}_{j}-\tilde{\boldsymbol{x}}_{j} \cdot h(\chi)\right\} \\
& \mathbb{P}\left(\boldsymbol{X}=\tilde{\boldsymbol{x}}_{j}, S=\tilde{s}_{j}, C=\tilde{c}_{j}, L^{\prime}=\tilde{\ell}_{j}+k, A=\beta\right) \\
\geq & (1-\delta) \sum_{\beta \in[T]} \sum_{j \in[J]} \tilde{s}_{j} \sum_{k \geq 0} \mathbf{1}\left\{k>\tilde{c}_{j}-\tilde{\ell}_{j}-\tilde{\boldsymbol{x}}_{j} \cdot h(\chi)\right\} p_{j}^{\beta} \\
& \mathbb{P}\left(\sum_{s \in[R]} s \operatorname{Poi}\left(\sum_{\gamma \in[T]} \tilde{w}_{j}^{-, s, \gamma} \hat{z}_{1}^{s, \beta, \gamma}\right)=k\right) \\
= & (1-\delta) \stackrel{\circ}{g}\left(\hat{z}_{1}, \chi\right)
\end{aligned}
$$

and possibly further reducing $\delta$, we derive $n^{-1} \hat{\mathcal{S}}_{n} \geq \sqrt{1-\epsilon} \stackrel{\circ}{g}_{\delta}\left(\hat{\chi}_{\delta}\right) \geq(1-\epsilon) \stackrel{\circ}{g}\left(\hat{z}_{1}, \hat{\chi}_{1}\right)$ w.h.p. So if $\left(\hat{z}_{1}, \hat{\chi}_{1}\right)=(\hat{z}, \hat{\chi})$, then this finishes the proof of the lower bounds.

If $\left(\hat{z}_{1}, \hat{\chi}_{1}\right) \neq(\hat{z}, \hat{\chi})$, then by construction of $(\hat{z}, \hat{\chi}), \hat{z}_{1}$ and $\hat{\chi}_{1}$ it must hold that $\hat{\chi}_{1} \leq \hat{\chi}$ and $\hat{z}_{1} \lesseqgtr \hat{z}_{2} \leq \hat{z}$, where $\hat{z}_{2} \in \mathbb{R}_{+, 0}^{V}$ denotes the smallest vector such that for all $(r, \alpha, \beta) \in V$,

$$
\mathbb{E}\left[W^{+, r, \alpha} \mathbb{P}\left(\sum_{s \in[R]} s \text { Poi }\left(\sum_{\gamma \in[T]} W^{-, s, \gamma} \hat{z}_{2}^{s, \beta, \gamma}\right)>C-L-\boldsymbol{X} \cdot h\left(\hat{\chi}_{1}\right)\right) \mathbf{1}\{A=\beta\}\right]=\hat{z}_{2}^{r, \alpha, \beta} .
$$

The next step in the cascade process would now be the default contagion cascade from (i') starting from the state of the system after the fire sales cascade. Note, however, that (w.h.p.) we can equivalently restart the whole cascade process if for the default contagion cascade we choose capitals $c_{i}-\ell_{i}-\boldsymbol{x}_{i} \cdot h\left((1-\epsilon) \hat{\chi}_{1}\right)$. If anything this reduces contagion effects which is alright because we are interested in lower bounds. 
By the finitariness of the system, if we choose $\epsilon$ small enough, then $\hat{z}_{2}$ is also the smallest solution of

$$
\begin{aligned}
& \mathbb{E}\left[W^{+, r, \alpha} \mathbb{P}\left(\sum_{s \in[R]} s \operatorname{Poi}\left(\sum_{\gamma \in[T]} W^{-, s, \gamma} \hat{z}_{2}^{s, \beta, \gamma}\right)>C-L-\boldsymbol{X} \cdot h\left((1-\epsilon) \hat{\chi}_{1}\right)\right) \mathbf{1}\{A=\beta\}\right] \\
& =\hat{z}_{2}^{r, \alpha, \beta} .
\end{aligned}
$$

and we can hence use [31, Theorem 3.2.4] to quantify the default cascade. By exactly the same means as above we can then translate the losses due to default contagion into exogenous losses and investigate the fire sales process by [18, Theorem EC. 1]. We derive that the vector of finally sold shares is lower bounded by $n(1-\epsilon) \hat{\chi}_{2}$ w.h.p., where $\hat{\chi}_{2}$ denotes the smallest joint root of the functions $f^{m}\left(\hat{z}_{2}, \chi\right)$ for $z=\hat{z}_{2}$, and $n^{-1} \hat{\mathcal{S}}_{n} \geq(1-\epsilon) \stackrel{\circ}{g}\left(\hat{z}_{2}, \hat{\chi}_{2}\right)$ w.h.p.

Again, if $\left(\hat{z}_{2}, \hat{\chi}_{2}\right)=(\hat{z}, \hat{\chi})$, then this finishes the proof of the lower bounds. Otherwise we can continue on for $t \geq 3$. Note, however, that $\hat{z} \geq \hat{z}_{t}$ is only possible if $\hat{\chi}_{t-1}$ and $\hat{\chi}_{t}$ are separated by a hyperplane of discontinuity of $f^{r, \alpha, \beta}$ for some $(r, \alpha, \beta) \in V$ (and hence the fire sales lead to further defaults in the system). However, as remarked earlier, there can only be finitely many such hyperplanes for finitary systems. Hence by the procedure outlined above, we will reach $\hat{z}$ in finitely many steps and hence the end results still hold w.h.p.

We can now turn to the second part of the proof. We consider the contagion process in rounds (i) and (ii) to derive upper bounds on $\overline{\mathcal{S}}_{n}$ and $\bar{\chi}_{n}$. Let $(\tilde{z}(\delta), \tilde{\chi}(\delta))_{\delta>0}$ be the constructing sequence of $\left(z^{*}, \chi^{*}\right)$ analogue to [18, Remark EC. 1]. Then note that by upper semi-continuity and the discrete nature of $f^{r, \alpha, \beta}$ we can find $\Delta>0$ such that $f^{r, \alpha, \beta}(z, \tilde{\chi}(\delta))=f^{r, \alpha, \beta}\left(z, \chi^{*}\right)$ for all $0 \leq \delta<\Delta,(r, \alpha, \beta) \in V$ and $z \in \mathbb{R}_{+, 0}^{V}$.

Fix now some $\delta \in(0, \Delta)$ and consider the financial system with reduced capital values $c_{i}-\ell_{i}-\boldsymbol{x}_{i} \cdot h(\tilde{\boldsymbol{\chi}}(\delta))$ for each bank $i \in[n]$. We only want to consider the default contagion process in this new financial system and we are hence in the setting of [16] with limiting random variables $\left(\boldsymbol{W}^{-}, \boldsymbol{W}^{+}, S,\lceil C-L-\boldsymbol{X} \cdot h(\tilde{\boldsymbol{\chi}}(\delta))\rceil^{+}, A\right)$. Note that by finitariness the regularity transfers. By the choice of $\delta$ above, we derive that for $z_{\delta}^{*}$ in this new financial system, it holds $z_{\delta}^{*}=z^{*}$ and by [31, Theorem 3.2.4] we know that the final systemic damage in the new system is upper bounded by

$$
\begin{aligned}
& n \sum_{\beta \in[T]} \mathbb{E}\left[S \mathbb{P}\left(\sum_{s \in[R]} s \text { Poi }\left(\sum_{\gamma \in[T]} W^{-, s, \gamma}\left(z^{*}\right)^{s, \beta, \gamma}\right) \geq C-L-\boldsymbol{X} \cdot h(\tilde{\chi}(\delta))\right) \mathbf{1}\{A=\beta\}\right] \\
& \quad+o_{p}(n) .
\end{aligned}
$$

The proof of [31, Theorem 3.2.4] actually shows that the number of banks of type $\beta$ and class $j$ with at least an edge-weight of $k$ from defaulted neighbors at the end of the default contagion process is upper bounded by

$$
(1+\epsilon) n p_{j}^{\beta} \mathbb{P}\left(\sum_{s \in[R]} s \text { Poi }\left(\sum_{\gamma \in[T]} \tilde{w}_{j}^{-, s, \gamma}\left(z^{*}\right)^{s, \beta, \gamma}\right) \geq k\right)
$$

w.h.p. for any fixed $\epsilon>0$. Similarly, as in the first part of this proof (for the lower bounds) we can then construct a fire sales system with limiting random vector $\left(\boldsymbol{X}, S, C, L^{\prime}\right)$ such that

$$
\begin{aligned}
\mathbb{P} & \left(\boldsymbol{X}=\tilde{\boldsymbol{x}}_{j}, S=\tilde{s}_{j}, C=\tilde{c}_{j}, L^{\prime}=\tilde{\ell}_{j}+k, A=\beta\right) \\
& =(1+\epsilon) p_{j}^{\beta} \mathbb{P}\left(\sum_{s \in[R]} s \operatorname{Poi}\left(\sum_{\gamma \in[T]} \tilde{w}_{j}^{-, s, \gamma}\left(z^{*}\right)^{s, \beta, \gamma}\right)=k\right),
\end{aligned}
$$




$$
\begin{aligned}
& \leq \leq k<\left\lceil\tilde{c}_{j}-\tilde{\ell}_{j}-\tilde{\boldsymbol{x}}_{j} \cdot h(\tilde{\boldsymbol{\chi}}(\delta))\right], \\
& \mathbb{P}\left(\boldsymbol{X}=\tilde{\boldsymbol{x}}_{j}, S=\tilde{s}_{j}, C=\tilde{c}_{j}, L^{\prime}=\tilde{\ell}_{j}+\left\lceil\tilde{c}_{j}-\tilde{\ell}_{j}-\tilde{\boldsymbol{x}}_{j} \cdot h(\tilde{\boldsymbol{\chi}}(\delta))\right], A=\beta\right) \\
&=p_{j}^{\beta}-\sum_{k=0}^{\left\lceil\tilde{c}_{j}-\tilde{\ell}_{j}-\tilde{\boldsymbol{x}}_{j} \cdot h(\tilde{\boldsymbol{\chi}}(\delta))\right]-1}(1+\epsilon) p_{j}^{\beta} \mathbb{P}\left(\sum_{s \in[R]} s \operatorname{Poi}\left(\sum_{\gamma \in[T]} \tilde{w}_{j}^{-, s, \gamma}\left(z^{*}\right)^{s, \beta, \gamma}\right)=k\right) \\
& \leq(1+\epsilon) p_{j}^{\beta} \mathbb{P}\left(\sum_{s \in[R]} s \operatorname{Poi}\left(\sum_{\gamma \in[T]} \tilde{w}_{j}^{-, s, \gamma}\left(z^{*}\right)^{s, \beta, \gamma}\right) \geq\left\lceil\tilde{c}_{j}-\tilde{\ell}_{j}-\tilde{\boldsymbol{x}}_{j} \cdot h(\tilde{\chi}(\delta))\right\rceil\right)
\end{aligned}
$$

which dominates the stochastic final state after the default contagion cascade w.h.p.

Let now

$$
f_{\epsilon}^{m}(\chi):=\sum_{\beta \in[T]} \mathbb{E}\left[X^{m} \rho_{\beta}^{m}\left(\frac{L^{\prime}+\boldsymbol{X} \cdot h(\chi)}{C}\right) \mathbf{1}\{A=\beta\}\right]-\chi^{m}
$$

the corresponding functions for the fire sales system as in [18] adapted to the setting with heterogeneous sales functions and $\chi_{\epsilon}^{*}$ the corresponding value for $\chi^{*}$ in [18]. Then for $f^{m}(z, \chi)$ as in Sect. 3,

$$
\begin{aligned}
& f_{\epsilon}^{m}(\chi)+\chi^{m} \\
& \quad=\sum_{\beta \in[T]} \sum_{j \in J} \sum_{k \geq 0} \tilde{x}_{j}^{m} \rho_{\beta}^{m}\left(\frac{\tilde{\ell}_{j}+k+\tilde{\boldsymbol{x}}_{j} \cdot h(\chi)}{\tilde{c}_{j}}\right) \mathbb{P}\left(\boldsymbol{X}=\tilde{\boldsymbol{x}}_{j}, C=\tilde{c}_{j}, L^{\prime}=\tilde{\ell}_{j}+k, A=\beta\right) \\
& \quad \leq \sum_{\beta \in[T]} \sum_{j \in J} \sum_{k \geq 0} \tilde{x}_{j}^{m} \rho_{\beta}^{m}\left(\frac{\tilde{\ell}_{j}+k+\tilde{\boldsymbol{x}}_{j} \cdot h(\chi)}{\tilde{c}_{j}}\right)(1+\epsilon) p_{j}^{\beta} \\
& \quad \mathbb{P}\left(\sum_{s \in[R]} s \operatorname{Poi}\left(\sum_{\gamma \in[T]} \tilde{w}_{j}^{-, s, \gamma}\left(z^{*}\right)^{s, \beta, \gamma}\right)=k\right) \\
& \quad=(1+\epsilon)\left(f^{m}\left(z^{*}, \chi\right)+\chi^{m}\right) .
\end{aligned}
$$

In particular, we can choose $\epsilon>0$ small enough such that

$f_{\epsilon}^{m}(\tilde{\chi}(\delta / 2)) \leq f^{m}\left(z^{*}, \tilde{\chi}(\delta / 2)\right)+\epsilon\left(f^{m}\left(z^{*}, \tilde{\chi}(\delta / 2)\right)+\tilde{\chi}^{m}(\delta / 2)\right) \leq-\delta / 2+\epsilon \mathbb{E}\left[X^{m}\right]<0$,

where in the last inequality it was used that $f^{m}\left(z^{*}, \tilde{\chi}(\delta / 2)\right) \leq f^{m}(\tilde{z}(\delta / 2), \tilde{\chi}(\delta / 2))=-\delta / 2$. We can hence conclude that $\chi_{\epsilon}^{*} \leq \tilde{\chi}(\delta / 2)$ componentwise. By (a slightly tedious but simple generalization to type dependent sales functions of) [18, Theorem EC. 1] we thus derive that the number of finally sold shares of asset $m$ in the fire sales system $\left(\boldsymbol{X}, S, C, L^{\prime}\right)$ is upper bounded by $n\left(\left(\chi_{\epsilon}^{*}\right)^{m}+o(1)\right) \leq n\left(\tilde{\chi}^{m}(\delta / 2)+o(1)\right) \leq n \tilde{\chi}^{m}(\delta)$, where the last inequality holds for $n$ large enough since $\tilde{\chi}^{m}(\delta)>0$.

The idea for the rest of this proof is now to apply this upper bound on the number of finally sold shares inductively in each step of the following adjusted contagion process: Let $\bar{\chi}_{0}=\mathbf{0} \in \mathbb{R}^{M}$ and for $1 \leq k$ choose $\overline{\mathcal{D}}_{k} \subseteq[n]$ the smallest set such that

$$
\overline{\mathcal{D}}_{k}=\left\{i \in[n]: \sum_{j \in \overline{\mathcal{D}}_{k}} e_{j, i} \geq c_{i}-\ell_{i}-\boldsymbol{x}_{i} \cdot h\left(\bar{\chi}_{k-1}\right)\right\}
$$


respectively $\bar{\chi}_{k} \in \mathbb{R}_{+, 0}^{M}$ the smallest vector such that

$$
\bar{\chi}_{k}=n^{-1} \sum_{i \in[n]} \boldsymbol{x}_{i} \odot \rho_{\alpha_{i}}\left(\frac{\sum_{j \in \overline{\mathcal{D}}_{k}} e_{j, i}+\ell_{i}+\boldsymbol{x}_{i} \cdot h\left(\bar{\chi}_{k}\right)}{c_{i}}\right) .
$$

Clearly, this process stabilizes after at most $n$ steps at $\left(\overline{\mathcal{D}}_{n}, \bar{\chi}_{n}\right)$, the smallest solution to (2.3) and (2.4) and by Lemma 2.1 gives thus an upper bound to the actual contagion process described by (2.1) and (2.2), which is sufficient for our considerations here.

In particular, since $\bar{\chi}_{0}=\mathbf{0} \leq \tilde{\chi}(\delta)$, we derive that $\overline{\mathcal{D}}_{1} \subseteq \mathcal{D}^{\delta}$, where $\mathcal{D}^{\delta} \subseteq[n]$ is the smallest set such that

$$
\mathcal{D}^{\delta}=\left\{i \in[n]: \sum_{j \in \mathcal{D}^{\delta}} e_{j, i} \geq c_{i}-\ell_{i}-\boldsymbol{x}_{i} \cdot h(\tilde{\chi}(\delta))\right\}
$$

and hence $\bar{\chi}_{1} \leq \chi^{\delta}$, where $\chi^{\delta}$ denotes the smallest vector such that

$$
\chi^{\delta}=n^{-1} \sum_{i \in[n]} \boldsymbol{x}_{i} \odot \rho_{\alpha_{i}}\left(\frac{\sum_{j \in \mathcal{D}^{\delta}} e_{j, i}+\ell_{i}+\boldsymbol{x}_{i} \cdot h\left(\chi^{\delta}\right)}{c_{i}}\right) .
$$

However, (8.4) is exactly the cascade of default contagion with initial capitals given by $c_{i}-\ell_{i}-\boldsymbol{x}_{i} \cdot h(\tilde{\chi}(\delta)), i \in[n]$, which we considered before and (8.5) the subsequent cascade of fire sales for which we showed that the vector of finally sold shares is upper bounded by $n \tilde{\chi}(\delta)$ w.h.p. (i.e. $\chi^{\delta} \leq \tilde{\chi}(\delta)$ ). We can then consider the second iteration and derive that w.h.p. $\overline{\mathcal{D}}_{2} \subseteq \mathcal{D}^{\delta}$ and $\bar{\chi}_{2} \leq \chi^{\delta}$. Inductively this shows that w.h.p. $\overline{\mathcal{D}}_{k} \subseteq \mathcal{D}^{\delta}$ and $\bar{\chi}_{k} \leq \chi^{\delta} \leq \tilde{\chi}(\delta)$ w.h.p. for each fixed $k \in \mathbb{N}$ (independent of $n$ ).

Now note that because of the finitariness of the system, the contagion process actually stabilizes already after a bounded number of iterations $K \in \mathbb{N}$ (independent of $n$ ). We have thus shown that also for the final vector of sold shares $\chi_{n}$ it holds $\chi_{n} \leq \bar{\chi}_{n}=\bar{\chi}_{K} \leq \tilde{\chi}(\delta)$ w.h.p. Letting $\delta \rightarrow 0$ this proves the upper bound on $\chi_{n}$.

For the final systemic damage note that w.h.p. $\mathcal{D}_{n} \subseteq \mathcal{D}^{\delta}$ and hence

$$
n^{-1} \mathcal{S}_{n} \leq n^{-1} \mathcal{S}^{\delta}:=n^{-1} \sum_{i \in \mathcal{D}^{\delta}} s_{i}
$$

But

$$
\begin{aligned}
n^{-1} \mathcal{S}^{\delta} & \leq \mathbb{E}\left[S \mathbb{P}\left(\sum_{s \in[R]} s \text { Poi }\left(\sum_{\gamma \in[T]} W^{-, s, \gamma}\left(z^{*}\right)^{s, \beta, \gamma}\right) \geq C-L-\boldsymbol{X} \cdot h(\tilde{\chi}(\delta))\right)\right]+o_{p}(1) \\
& =g\left(z^{*}, \tilde{\chi}(\delta)\right)+o_{p}(1)
\end{aligned}
$$

by (8.3). Using upper semi-continuity of $g$ and letting $\delta \rightarrow 0$ this finishes the proof.

\subsection{Proof of Theorem 3.3}

In this section, we show how the validity of Theorem 8.2 can be extended to the case of general (non-finitary) regular vertex sequences. The idea is the following: We will approximate the given regular vertex sequence from below and from above by finitary vertex sequences and couple the contagion processes in those systems such that the final default fraction and the number of sold shares is under- or overestimated by the finitary systems. 
We will describe the finitary systems by their distribution functions $\left\{F_{k}^{A}\right\}_{k \in \mathbb{N}}$ and $\left\{F_{k}^{B}\right\}_{k \in \mathbb{N}}$ respectively in the following and we need to ensure that the functions $g$ and $f^{m}, m \in[M]$, are approximated close enough. To this end, consider the integrands

$$
\begin{aligned}
& h_{g}\left(\boldsymbol{z}, \boldsymbol{\chi} ; \boldsymbol{w}^{-}, \boldsymbol{w}^{+}, \boldsymbol{x}, s, c, \ell, \tau\right) \\
& \quad:=s \sum_{\beta \in[T]} \psi\left(\sum_{\gamma \in[T]} w^{-, 1, \gamma} z^{1, \beta, \gamma}, \ldots, \sum_{\gamma \in[T]} w^{-, R, \gamma} z^{R, \beta, \gamma} ; c-l-\boldsymbol{x} \cdot h(\chi)\right) \mathbf{1}\{\tau=\beta\}, \\
& h_{f}^{r, \alpha, \beta}\left(\boldsymbol{z}, \boldsymbol{\chi} ; \boldsymbol{w}^{-}, \boldsymbol{w}^{+}, \boldsymbol{x}, s, c, \ell, \tau\right) \\
& \quad:=w^{+, r, \alpha} \psi\left(\sum_{\gamma \in[T]} w^{-, 1, \gamma} z^{1, \beta, \gamma}, \ldots, \sum_{\gamma \in[T]} w^{-, R, \gamma} z^{R, \beta, \gamma} ; c-l-\boldsymbol{x} \cdot h(\chi)\right) \\
& \quad \mathbf{1}\{\tau=\beta\},(r, \alpha, \beta) \in V, \\
& h_{f}^{m}\left(\boldsymbol{z}, \boldsymbol{\chi} ; \boldsymbol{w}^{-}, \boldsymbol{w}^{+}, \boldsymbol{x}, s, c, \ell, \tau\right) \\
& \quad:=x^{m} \sum_{\beta \in[T]} \phi_{\beta}^{m}\left(\sum_{\gamma \in[T]} w^{-, 1, \gamma} z^{1, \beta, \gamma}, \ldots, \sum_{\gamma \in[T]} w^{-, R, \gamma} z^{R, \beta, \gamma} ; l+\boldsymbol{x} \cdot h(\chi), c\right) \\
& \quad \mathbf{1}\{\tau=\beta\}, m \in[M],
\end{aligned}
$$

for $\left(\boldsymbol{z}, \boldsymbol{\chi}, \boldsymbol{w}^{-}, \boldsymbol{w}^{+}, \boldsymbol{x}, s, c, \ell, \tau\right) \in \mathbb{R}_{+, 0}^{V} \times \mathbb{R}_{+, 0}^{M} \times D_{\infty}$, with $\boldsymbol{w}^{ \pm}=\left(w^{ \pm, r, \alpha}\right)_{r \in[R], \alpha \in[T]}$ as well as $D_{\infty}:=\left(\mathbb{R}_{+, 0}^{[R] \times[T]}\right)^{2} \times \mathbb{R}_{+, 0}^{M+3} \times[T]$ and where $\psi$ and $\phi_{\beta}, \beta \in[T]$, are as defined in Sect. 3. Then

$$
\begin{aligned}
g(z, \chi) & =\int_{D_{\infty}} h_{g}\left(z, \chi ; \boldsymbol{w}^{-}, \boldsymbol{w}^{+}, \boldsymbol{x}, s, c, \ell, \tau\right) \mathrm{d} F\left(\boldsymbol{w}^{-}, \boldsymbol{w}^{+}, \boldsymbol{x}, s, c, \ell, \tau\right), \\
f^{r, \alpha, \beta}(z, \chi) & =\int_{D_{\infty}} h_{f}^{r, \alpha, \beta}\left(z, \chi ; \boldsymbol{w}^{-}, \boldsymbol{w}^{+}, \boldsymbol{x}, s, c, \ell, \tau\right) \mathrm{d} F\left(\boldsymbol{w}^{-}, \boldsymbol{w}^{+}, \boldsymbol{x}, s, c, \ell, \tau\right)-z^{r, \alpha, \beta},(r, \alpha, \beta) \in V, \\
f^{m}(z, \chi) & =\int_{D_{\infty}} h_{f}^{m}\left(z, \chi ; \boldsymbol{w}^{-}, \boldsymbol{w}^{+}, \boldsymbol{x}, s, c, \ell, \tau\right) \mathrm{d} F\left(\boldsymbol{w}^{-}, \boldsymbol{w}^{+}, \boldsymbol{x}, s, c, \ell, \tau\right)-\chi^{m}, m \in[M],
\end{aligned}
$$

where $F$ denotes the distribution function of $\left(\boldsymbol{W}^{-}, \boldsymbol{W}^{+}, \boldsymbol{X}, S, C, L, A\right)$ (note that the integrands vanish for $c=\infty$ and it is thus sufficient to integrate over $D_{\infty}$ ). We denote in the following

$$
H:=\left\{h_{g}\right\} \cup \bigcup_{(r, \alpha, \beta) \in V}\left\{h_{f}^{r, \alpha, \beta}\right\} \cup \bigcup_{m \in[M]}\left\{h_{f}^{m}\right\}
$$

and $Z:=[\mathbf{0}, \zeta] \times[\mathbf{0}, \eta] \subset \mathbb{R}_{+, 0}^{V} \times \mathbb{R}_{+, 0}^{M}$, for $\zeta^{r, \alpha, \beta}=\mathbb{E}\left[W^{+, r, \alpha} \mathbf{1}\{A=\beta\}\right],(r, \alpha, \beta) \in V$, and $\eta^{m}=\mathbb{E}\left[X^{m}\right], m \in[M]$. If we further let

$$
\begin{aligned}
& \stackrel{\circ}{h}_{g}\left(\boldsymbol{z}, \boldsymbol{\chi} ; \boldsymbol{w}^{-}, \boldsymbol{w}^{+}, \boldsymbol{x}, s, c, \ell, \tau\right) \\
& \quad:=s \sum_{\beta \in[T]} \stackrel{\circ}{\psi}\left(\sum_{\gamma \in[T]} w^{-, 1, \gamma} z^{1, \beta, \gamma}, \ldots, \sum_{\gamma \in[T]} w^{-, R, \gamma} z^{R, \beta, \gamma} ; c-l-\boldsymbol{x} \cdot h(\chi)\right) \mathbf{1}\{\tau=\beta\}, \\
& \stackrel{\circ}{h}^{r}, \alpha, \beta_{f}\left(\boldsymbol{z}, \boldsymbol{\chi} ; \boldsymbol{w}^{-}, \boldsymbol{w}^{+}, \boldsymbol{x}, s, c, \ell, \tau\right) \\
& \quad:=w^{+, r, \alpha} \stackrel{\circ}{\psi}\left(\sum_{\gamma \in[T]} w^{-, 1, \gamma} z^{1, \beta, \gamma}, \ldots, \sum_{\gamma \in[T]} w^{-, R, \gamma} z^{R, \beta, \gamma} ; c-l-\boldsymbol{x} \cdot h(\chi)\right)
\end{aligned}
$$




$$
\begin{aligned}
& \quad \mathbf{1}\{\tau=\beta\},(r, \alpha, \beta) \in V, \\
& \stackrel{\circ}{h}_{f}^{m}\left(\boldsymbol{z}, \boldsymbol{\chi} ; \boldsymbol{w}^{-}, \boldsymbol{w}^{+}, \boldsymbol{x}, s, c, \ell, \tau\right) \\
& :=x^{m} \sum_{\beta \in[T]} \stackrel{\circ}{\phi}_{\beta}^{m}\left(\sum_{\gamma \in[T]} w^{-, 1, \gamma} z^{1, \beta, \gamma}, \ldots, \sum_{\gamma \in[T]} w^{-, R, \gamma} z^{R, \beta, \gamma} ; l+\boldsymbol{x} \cdot h(\chi), c\right) \\
& \quad \mathbf{1}\{\tau=\beta\}, m \in[M],
\end{aligned}
$$

where $\stackrel{\circ}{\psi}$ and $\stackrel{\circ}{\phi}_{\beta}, \beta \in[T]$, are defined as in Sect. 3, then it holds

$$
\begin{aligned}
\stackrel{\circ}{g}(z, \chi)= & \int_{D_{\infty}} \stackrel{\circ}{h}\left(z, \chi ; \boldsymbol{w}^{-}, \boldsymbol{w}^{+}, \boldsymbol{x}, s, c, \ell, \tau\right) \mathrm{d} F\left(\boldsymbol{w}^{-}, \boldsymbol{w}^{+}, \boldsymbol{x}, s, c, \ell, \tau\right), \\
f^{r, \alpha, \beta}(z, \chi)= & \int_{D_{\infty}} \stackrel{\circ}{h^{r}}, \alpha, \beta_{f}\left(z, \chi ; \boldsymbol{w}^{-}, \boldsymbol{w}^{+}, \boldsymbol{x}, s, c, \ell, \tau\right) \mathrm{d} F\left(\boldsymbol{w}^{-}, \boldsymbol{w}^{+}, \boldsymbol{x}, s, c, \ell, \tau\right) \\
& -z^{r, \alpha, \beta},(r, \alpha, \beta) \in V, \\
\stackrel{\circ}{f}^{m}(z, \chi)= & \int_{D_{\infty}} \stackrel{\circ}{h_{f}^{m}}\left(z, \chi ; \boldsymbol{w}^{-}, \boldsymbol{w}^{+}, \boldsymbol{x}, s, c, \ell, \tau\right) \mathrm{d} F\left(\boldsymbol{w}^{-}, \boldsymbol{w}^{+}, \boldsymbol{x}, s, c, \ell, \tau\right)-\chi^{m}, m \in[M] .
\end{aligned}
$$

Also denote $\stackrel{\circ}{H}:=\left\{\stackrel{\circ}{h}_{g}\right\} \cup \bigcup_{(r, \alpha, \beta) \in V}\left\{\stackrel{\circ}{h}^{r}, \alpha, \beta_{f}\right\} \cup \bigcup_{m \in[M]_{0}}\left\{\stackrel{\circ}{h}_{f}^{m}\right\}$.

For $j \in \mathbb{N}$, consider now discretizations

$$
\begin{aligned}
& \tilde{F}_{j}^{A}\left(\boldsymbol{w}^{-}, \boldsymbol{w}^{+}, \boldsymbol{x}, s, c, \ell, \tau\right):=F\left(\frac{\left\lceil j \boldsymbol{w}^{-}\right\rceil}{j}, \frac{\left\lceil j \boldsymbol{w}^{+}\right\rceil}{j}, \frac{\lceil j \boldsymbol{x}\rceil}{j}, \frac{\lfloor j s\rfloor}{j}, \frac{\lfloor j c\rfloor}{j}, \frac{\lceil j \ell\rceil}{j}, \tau\right), \\
& \tilde{F}_{j}^{B}\left(\boldsymbol{w}^{-}, \boldsymbol{w}^{+}, \boldsymbol{x}, s, c, \ell, \tau\right):=F\left(\frac{\left\lfloor j \boldsymbol{w}^{-}\right\rfloor}{j}, \frac{\left\lfloor j \boldsymbol{w}^{+}\right\rfloor}{j}, \frac{\lfloor j \boldsymbol{x}\rfloor}{j}, \frac{\lceil j s\rceil}{j}, \frac{\lceil j c\rceil}{j}, \frac{\lfloor j \ell\rfloor}{j}, \tau\right),
\end{aligned}
$$

where $\lceil\cdot\rceil$ and $\lfloor\cdot\rfloor$ shall be applied componentwise on the vectors $j \boldsymbol{w}^{-}, j \boldsymbol{w}^{+}$and $j \boldsymbol{x}$. In particular, the distributions $\left\{\tilde{F}_{j}^{A, B}\right\}_{j \in \mathbb{N}}$ converge to $F$. Choose now $k \in \mathbb{N}, h \in H$ and let for $0 \leq s \leq 2 k^{2}$ the sets

$$
I_{s}(z, \chi):=\left\{\left(\boldsymbol{w}^{-}, \boldsymbol{w}^{+}, \boldsymbol{x}, s, c, \ell, \tau\right): h\left(z, \chi ; \boldsymbol{w}^{-}, \boldsymbol{w}^{+}, \boldsymbol{x}, s, c, \ell, \tau\right) \geq \frac{s}{2 k}\right\}
$$

which are closed by upper semi-continuity of $h$. Further, let

$$
\hat{h}\left(z, \chi ; \boldsymbol{w}^{-}, \boldsymbol{w}^{+}, \boldsymbol{x}, s, c, \ell, \tau\right):=\frac{1}{2 k} \sum_{s=0}^{2 k^{2}} \mathbf{1}\left\{\left(\boldsymbol{w}^{-}, \boldsymbol{w}^{+}, \boldsymbol{x}, s, c, \ell, \tau\right) \in I_{s}(z, \chi)\right\}
$$

such that in particular $h-(2 k)^{-1} \leq \hat{h} \leq h \leq k$ on $\mathbb{R}_{+, 0}^{V} \times \mathbb{R}_{+, 0}^{M} \times D_{k}$ with

$$
D_{k}:=\left\{\left(\boldsymbol{w}^{-}, \boldsymbol{w}^{+}, \boldsymbol{x}, s, c, \ell, \tau\right): \boldsymbol{w}^{-} \leq k \mathbf{1}, \boldsymbol{w}^{+} \leq k \mathbf{1}, \boldsymbol{x} \leq k \mathbf{1}, s \leq k, c \leq k, \ell \leq k\right\} .
$$

By the Portmanteau theorem, we then know that for $j \geq j_{k}$ large enough it holds

$$
\int_{D_{k}} \hat{h} \mathrm{~d} \tilde{F}_{j}^{A, B}-\int_{D_{k}} \hat{h} \mathrm{~d} F \leq \frac{1}{2 k}
$$

and hence

$$
\int_{D_{k}} h\left(z, \chi ; \boldsymbol{w}^{-}, \boldsymbol{w}^{+}, \boldsymbol{x}, s, c, \ell, \tau\right) \mathrm{d} \tilde{F}_{j}^{A, B}-\int_{D_{k}} h\left(z, \chi ; \boldsymbol{w}^{-}, \boldsymbol{w}^{+}, \boldsymbol{x}, s, c, \ell, \tau\right) \mathrm{d} F \leq k^{-1}
$$


Completely analogue, but choosing

$$
\stackrel{\circ}{I}_{s}(z, \chi):=\left\{\left(\boldsymbol{w}^{-}, \boldsymbol{w}^{+}, \boldsymbol{x}, s, c, \ell, \tau\right): \stackrel{\circ}{h}\left(z, \chi ; \boldsymbol{w}^{-}, \boldsymbol{w}^{+}, \boldsymbol{x}, s, c, \ell, \tau\right)>\frac{s}{2 k}\right\},
$$

we derive for $\stackrel{\circ}{h} \in \stackrel{\circ}{H}$ and $j \geq j_{k}$ (possibly increase $j_{k}$ ) that

$$
\int_{D_{D_{k}}} \stackrel{\circ}{h}\left(\boldsymbol{z}, \boldsymbol{\chi} ; \boldsymbol{w}^{-}, \boldsymbol{w}^{+}, \boldsymbol{x}, s, c, \ell, \tau\right) \mathrm{d} \tilde{F}_{j}^{A, B}-\int_{\stackrel{\circ}{k}} \stackrel{\circ}{h}\left(\boldsymbol{z}, \boldsymbol{\chi} ; \boldsymbol{w}^{-}, \boldsymbol{w}^{+}, \boldsymbol{x}, s, c, \ell, \tau\right) \mathrm{d} F \geq-k^{-1},
$$

where $\stackrel{\circ}{D}_{k}:=\left\{\left(\boldsymbol{w}^{-}, \boldsymbol{w}^{+}, \boldsymbol{x}, s, c, \ell, \tau\right): \boldsymbol{w}^{-}<k \mathbf{1}, \boldsymbol{w}^{+}<k \mathbf{1}, \boldsymbol{x}<k \mathbf{1}, s<k, c<k, \ell<k\right\}$. We denote $\bar{F}_{k}^{A}:=\tilde{F}_{j_{k}}^{A}$ and $\bar{F}_{k}^{B}:=\tilde{F}_{j_{k}}^{B}$ in the following.

Note that $\bar{F}_{k}^{A, B}$ already describe discrete distributions approximating $F$ from below and from above. To qualify as a distribution function of a finitary vertex sequence, however, only finitely many atoms are allowed. For the lower bound, we thus choose

$$
F_{k}^{A}\left(\boldsymbol{w}^{-}, \boldsymbol{w}^{+}, \boldsymbol{x}, s, c, \ell, \tau\right):= \begin{cases}\bar{F}_{k}^{A}\left(\boldsymbol{w}^{-} \wedge k, \boldsymbol{w}^{+} \wedge k, \boldsymbol{x} \wedge k, s \wedge k, c \wedge k, \ell \wedge k, \tau\right), & \text { if } c<\infty, \\ \mathbf{1}, & \text { else, }\end{cases}
$$

thus setting capital to $\infty$ for banks with in-weight, out-weight, asset holdings, capital or exogenous losses larger than $k$. We call such banks large in the following. Banks with infinite capital keep their capital. As banks with infinite capital cannot ever default or sell any asset shares anyway we set their weights, asset holdings and losses all to zero. In particular, above choice reduces contagion in the system even further and thus for all $k \in \mathbb{N}$ the final systemic damage $n^{-1}\left(\mathcal{S}_{k}^{A}\right)_{n}$ is stochastically dominated by $n^{-1} \mathcal{S}_{n}$. The same holds for the number of finally sold shares of the assets.

We now want to construct the upper bound distribution function $F_{k}^{B}$. That is, we need to accumulate the contagious potential of all large banks to finitely many point masses. Thus denote the fraction of large $\beta$-banks in the system by

$$
\gamma_{k}^{\beta}:=\int_{D_{k}^{c}} \mathbf{1}\{\tau=\beta\} \mathrm{d} F\left(\boldsymbol{w}^{-}, \boldsymbol{w}^{+}, \boldsymbol{x}, s, c, \ell, \tau\right),
$$

where $D_{k}^{c}:=D_{\infty} \backslash D_{k}$, and

$$
\begin{aligned}
\left(\bar{w}_{k}^{\beta}\right)^{r, \alpha}: & =\left\{\begin{array}{ll}
2\left(\gamma_{k}^{\beta}\right)^{-1} \int_{D_{k}^{c}} w^{+, r, \alpha} \mathbf{1}\{\tau=\beta\} \mathrm{d} F\left(\boldsymbol{w}^{-}, \boldsymbol{w}^{+}, \boldsymbol{x}, s, c, \ell, \tau\right) \geq 2 k, & \text { if } \gamma_{k}^{\beta}>0, \\
2 k, & \text { if } \gamma_{k}^{\beta}=0,
\end{array} \quad(r, \alpha, \beta) \in V,\right. \\
\left(\bar{x}_{k}^{\beta}\right)^{m}: & =\left\{\begin{array}{ll}
2\left(\gamma_{k}^{\beta}\right)^{-1} \int_{D_{k}^{c}} x^{m} \mathbf{1}\{\tau=\beta\} \mathrm{d} F\left(\boldsymbol{w}^{-}, \boldsymbol{w}^{+}, \boldsymbol{x}, s, c, \ell, \tau\right) \geq 2 k, & \text { if } \gamma_{k}^{\beta}>0, \\
2 k, & \text { if } \gamma_{k}^{\beta}=0,
\end{array} \quad m \in[M],\right. \\
\bar{s}_{k}^{\beta}: & = \begin{cases}2\left(\gamma_{k}^{\beta}\right)^{-1} \int_{D_{k}^{c}} \boldsymbol{s} \mathbf{1}\{\tau=\beta\} \mathrm{d} F\left(\boldsymbol{w}^{-}, \boldsymbol{w}^{+}, \boldsymbol{x}, s, c, \ell, \tau\right) \geq 2 k, & \text { if } \gamma_{k}^{\beta}>0, \\
2 k, & \text { if } \gamma_{k}^{\beta}=0 .\end{cases}
\end{aligned}
$$

Similar as for the lower bound before, we now let $F_{k}^{B}$ be given by $\bar{F}_{k}^{B}$ on $D_{k}$. Moreover, let $F_{k}^{B}$ assign the remaining masses $\gamma_{k}^{\beta}$ to the points $\left(\mathbf{0}, \overline{\boldsymbol{w}}_{k}^{\beta}, \overline{\boldsymbol{x}}_{k}^{\beta}, \bar{s}_{k}^{\beta}, 0,0, \beta\right)$. As we have left out institutions with infinite capital thus far, finally let $F_{k}^{B}$ assign masses $\mathbb{P}(C=\infty, A=\beta)$ to the point $(\mathbf{0}, \mathbf{0}, \mathbf{0}, 0, \infty, 0, \beta)$ for each $\beta \in[T]$.

The construction above ensures that small banks are more contagious than in the original system as their weights, asset holdings and losses are increased whereas their capitals are 
decreased. Moreover, all large banks are initially defaulted and their total number of shares held of each asset $m$ is given by

$$
n \sum_{\beta \in[T]}\left(\bar{x}_{k}^{\beta}\right)^{m}\left(\gamma_{k}^{\beta}+o(1)\right)=2 n \int_{D_{k}^{c}} x^{m} \mathrm{~d} F\left(\boldsymbol{w}^{-}, \boldsymbol{w}^{+}, \boldsymbol{x}, s, c, \ell, \tau\right)(1+o(1))
$$

which is larger than in the original system,

$$
n \int_{D_{k}^{c}} x^{m} \mathrm{~d} F\left(\boldsymbol{w}^{-}, \boldsymbol{w}^{+}, \boldsymbol{x}, s, c, \ell, \tau\right)(1+o(1)) .
$$

Finally, also the total $r$-out-weight of large $\beta$-banks with respect to each type $\alpha \in[T]$

$$
n\left(\bar{w}_{k}^{\beta}\right)^{r, \alpha}\left(\gamma_{k}^{\beta}+o(1)\right)=2 n \int_{D_{k}^{c}} w^{+, r, \alpha} \mathbf{1}\{\tau=\beta\} \mathrm{d} F\left(\boldsymbol{w}^{-}, \boldsymbol{w}^{+}, \boldsymbol{x}, s, c, \ell, \tau\right)(1+o(1))
$$

is increased by approximation $F_{k}^{B}$ compared to the original system with

$$
n \int_{D_{k}^{c}} w^{+, r, \alpha} \mathbf{1}\{\tau=\beta\} \mathrm{d} F\left(\boldsymbol{w}^{-}, \boldsymbol{w}^{+}, \boldsymbol{x}, s, c, \ell, \tau\right)(1+o(1)) .
$$

Similar as in [16] for each $r \in[R]$ the number of $r$-edges from large banks to a specific small bank in the approximating system thus stochastically dominates their analogue in the original system and in particular this property transfers to the total direct exposure from large banks by summing over all $r \in[R]$. Altogether we derive the following result.

Lemma 8.3 Consider a regular vertex sequence and let sequences $\left\{F_{k}^{A}\right\}_{k \in \mathbb{N}}$ and $\left\{F_{k}^{B}\right\}_{k \in \mathbb{N}}$ be constructed as above. Further let $\left(\mathcal{S}_{k}^{A}\right)_{n}$ and $\left(\mathcal{S}_{k}^{B}\right)_{n}$ be the total systemic importance of finally defaulted institutions in the finitary approximating systems. Then it holds that

$$
n^{-1}\left(\mathcal{S}_{k}^{A}\right)_{n} \preceq n^{-1} \mathcal{S}_{n} \preceq n^{-1}\left(\mathcal{S}_{k}^{B}\right)_{n},
$$

where $\preceq$ denotes stochastic domination. If further $\left(\chi_{k}^{A, B}\right)_{n}^{m}$ denotes the number of finally sold shares of asset $m$ divided by $n$, then it holds

$$
\left(\chi_{k}^{A}\right)_{n}^{m} \preceq \chi_{n}^{m} \preceq\left(\chi_{k}^{B}\right)_{n}^{m} .
$$

Denote

$$
\begin{aligned}
g_{k}^{A, B}(z, \chi) & =\int_{D_{\infty}} h_{g}\left(z, \chi ; \boldsymbol{w}^{-}, \boldsymbol{w}^{+}, \boldsymbol{x}, s, c, \ell, \tau\right) \mathrm{d} F_{k}^{A, B}\left(\boldsymbol{w}^{-}, \boldsymbol{w}^{+}, \boldsymbol{x}, s, c, \ell, \tau\right), \\
\left(f_{k}^{A, B}\right)^{r, \alpha, \beta}(z, \chi) & =\int_{D_{\infty}} h_{f}^{r, \alpha, \beta}\left(z, \boldsymbol{\chi} ; \boldsymbol{w}^{-}, \boldsymbol{w}^{+}, \boldsymbol{x}, s, c, \ell, \tau\right) \mathrm{d} F_{k}^{A, B}\left(\boldsymbol{w}^{-}, \boldsymbol{w}^{+}, \boldsymbol{x}, s, c, \ell, \tau\right)-z^{r, \alpha, \beta}, \\
\left(f_{k}^{A, B}\right)^{m}(z, \chi) & =\int_{D_{\infty}} h_{f}^{m}\left(z, \boldsymbol{\chi} ; \boldsymbol{w}^{-}, \boldsymbol{w}^{+}, \boldsymbol{x}, s, c, \ell, \tau\right) \mathrm{d} F_{k}^{A, B}\left(\boldsymbol{w}^{-}, \boldsymbol{w}^{+}, \boldsymbol{x}, s, c, \ell, \tau\right)-\chi^{m},
\end{aligned}
$$

analogue to $g, f^{r, \alpha, \beta},(r, \alpha, \beta) \in V$, and $f^{m}, m \in[M]$. Moreover, let $\stackrel{\circ}{g}_{k}^{A, B},\left(\stackrel{\circ}{f^{A}}, B_{k}\right)^{r, \alpha, \beta}$, $\left(\stackrel{\circ}{f}^{A}, B_{k}\right)^{m},\left(\hat{z}_{k}^{A, B}, \hat{\chi}_{k}^{A, B}\right)$ and $\left(\left(z^{*}\right)_{k}^{A, B},\left(\chi^{*}\right)_{k}^{A, B}\right)$ the analogues of $\stackrel{\circ}{g}, f^{r, \alpha, \beta},(r, \alpha, \beta) \in V$, $f^{m}, m \in[M],(\hat{z}, \hat{\chi})$ and $\left(z^{*}, \chi^{*}\right)$. Then by Theorem 8.2 we derive lower and upper bounds for the approximating systems in terms of those quantities. The following lemma compares them to the original quantities. 
Lemma 8.4 It holds

$$
\liminf _{k \rightarrow \infty} \stackrel{\circ}{g}_{k}^{A}\left(\hat{z}_{k}^{A}, \hat{\chi}_{k}^{A}\right) \geq \stackrel{\circ}{g}(\hat{z}, \hat{\chi})
$$

and

$$
\limsup _{k \rightarrow \infty} g_{k}^{B}\left(\left(z^{*}\right)_{k}^{B},\left(\chi^{*}\right)_{k}^{B}\right) \leq g\left(z^{*}, \chi^{*}\right)
$$

as well as $\lim \sup _{k \rightarrow \infty}\left(\hat{\chi}_{k}^{A}\right)^{m} \geq \hat{\chi}^{m}$ and $\lim _{\inf _{k \rightarrow \infty}}\left(\left(\chi_{k}^{B}\right)^{*}\right)^{m} \leq\left(\chi^{*}\right)^{m}$ for all $m \in[M]$.

Proof. For $\stackrel{\circ}{h} \in \stackrel{\circ}{H}$, using (8.7) we derive

$$
\begin{aligned}
& \int_{{\stackrel{\circ}{D_{k}}}} \stackrel{\circ}{h}\left(\boldsymbol{z}, \boldsymbol{\chi} ; \boldsymbol{w}^{-}, \boldsymbol{w}^{+}, \boldsymbol{x}, s, c, \ell, \tau\right) \mathrm{d} F_{k}^{A}-\int_{{\stackrel{\circ}{D_{k}}}^{\circ}} \stackrel{\circ}{h}\left(\boldsymbol{z}, \boldsymbol{\chi} ; \boldsymbol{w}^{-}, \boldsymbol{w}^{+}, \boldsymbol{x}, s, c, \ell, \tau\right) \mathrm{d} F \\
& =\int_{{\stackrel{\circ}{D_{k}}}^{\prime}} \stackrel{\circ}{h}\left(\boldsymbol{z}, \boldsymbol{\chi} ; \boldsymbol{w}^{-}, \boldsymbol{w}^{+}, \boldsymbol{x}, s, c, \ell, \tau\right) \mathrm{d} \tilde{F}_{j_{k}}^{A}-\int_{{\stackrel{\circ}{D_{k}}}^{\circ}} \stackrel{\circ}{h}\left(\boldsymbol{z}, \boldsymbol{\chi} ; \boldsymbol{w}^{-}, \boldsymbol{w}^{+}, \boldsymbol{x}, s, c, \ell, \tau\right) \mathrm{d} F \\
& \geq-k^{-1} \rightarrow 0, \quad \text { as } k \rightarrow \infty \text {. }
\end{aligned}
$$

Moreover, $\int_{\stackrel{\circ}{k}_{k}^{c}} s \mathrm{~d} F \rightarrow 0, \int_{D_{k}^{c}} w^{+, r, \alpha} \mathrm{d} F \rightarrow 0,(r, \alpha) \in[R] \times[T]$, and $\int_{D_{k}^{c}} x^{m} \mathrm{~d} F \rightarrow 0, m \in$ $[M]$, as $k \rightarrow \infty$. In particular, it must then hold that $\int_{\stackrel{\circ}{D}_{k}^{c}} \stackrel{\circ}{h}\left(z, \chi ; w^{-}, w^{+}, \boldsymbol{x}, s, c, \ell, \tau\right) \mathrm{d} F \rightarrow$ 0 . Together with

$\int_{\stackrel{\circ}{k}^{c}} \stackrel{\circ}{h}\left(z, \chi ; \boldsymbol{w}^{-}, \boldsymbol{w}^{+}, \boldsymbol{x}, s, c, \ell, \tau\right) \mathrm{d} F_{k}^{A}=\int_{D_{k} \cap \stackrel{\circ}{D_{k}^{c}}} \stackrel{\circ}{h}\left(\boldsymbol{z}, \boldsymbol{\chi} ; \boldsymbol{w}^{-}, \boldsymbol{w}^{+}, \boldsymbol{x}, s, c, \ell, \tau\right) \mathrm{d} F_{k}^{A}=o(1)$, we can then conclude that

$$
\int_{D_{\infty}} \stackrel{\circ}{h}\left(z, \chi ; \boldsymbol{w}^{-}, \boldsymbol{w}^{+}, \boldsymbol{x}, s, c, \ell, \tau\right) \mathrm{d} F_{k}^{A}-\int_{D_{\infty}} \stackrel{\circ}{h}\left(z, \chi ; \boldsymbol{w}^{-}, \boldsymbol{w}^{+}, \boldsymbol{x}, s, c, \ell, \tau\right) \mathrm{d} F \geq o(1) .
$$

For $\left\{F_{k}^{B}\right\}_{k \in \mathbb{N}}$, we further obtain

$$
\begin{aligned}
& \int_{D_{k}^{c}} s \mathrm{~d} F_{k}^{B}\left(\boldsymbol{w}^{-}, \boldsymbol{w}^{+}, \boldsymbol{x}, s, c, \ell, \tau\right)=\sum_{\beta \in[T]} \bar{s}_{k}^{\beta} \gamma_{k}^{\beta}, \\
& \int_{D_{k}^{c}} w^{+, r, \alpha} \mathbf{1}\{\tau=\beta\} \mathrm{d} F_{k}^{B}\left(\boldsymbol{w}^{-}, \boldsymbol{w}^{+}, \boldsymbol{x}, s, c, \ell, \tau\right)=\left(\bar{w}_{k}^{\beta}\right)^{r, \alpha} \gamma_{k}^{\beta}, \\
& \int_{D_{k}^{c}} x^{m} \mathrm{~d} F_{k}^{B}\left(\boldsymbol{w}^{-}, \boldsymbol{w}^{+}, \boldsymbol{x}, s, c, \ell, \tau\right)=\sum_{\beta \in[T]}\left(\bar{x}_{k}^{\beta}\right)^{m} \gamma_{k}^{\beta}
\end{aligned}
$$

and as $k \rightarrow \infty$, by definition of $\gamma_{k}^{\beta}, \bar{s}_{k}^{\beta},\left(\bar{w}_{k}^{\beta}\right)^{r, \alpha}$ and $\left(\bar{x}_{k}^{\beta}\right)^{m}$ all those terms vanish. In particular,

$$
\int_{D_{k}^{c}} h\left(z, \chi ; \boldsymbol{w}^{-}, \boldsymbol{w}^{+}, \boldsymbol{x}, s, c, \ell, \tau\right) \mathrm{d} F_{k}^{B}\left(\boldsymbol{w}^{-}, \boldsymbol{w}^{+}, \boldsymbol{x}, s, c, \ell, \tau\right) \rightarrow 0, \quad \text { as } k \rightarrow \infty
$$

and by (8.6)

$$
\int_{D_{\infty}} h\left(z, \boldsymbol{\chi} ; \boldsymbol{w}^{-}, \boldsymbol{w}^{+}, \boldsymbol{x}, s, c, \ell, \tau\right) \mathrm{d} F_{k}^{B}-\int_{D_{\infty}} h\left(z, \boldsymbol{\chi} ; \boldsymbol{w}^{-}, \boldsymbol{w}^{+}, \boldsymbol{x}, s, c, \ell, \tau\right) \mathrm{d} F \leq o(1) .
$$


By (8.8) we can apply [18, Lemma EC. 2] (extend it by the $z$-dimensions) and thus derive that $\liminf _{k \rightarrow \infty} \hat{z}_{k}^{A} \geq \hat{z}$ and $\liminf _{k \rightarrow \infty} \hat{\chi}_{k}^{A} \geq \hat{\chi}$, where $\left(\hat{z}_{k}^{A}, \hat{\chi}_{k}^{A}\right)$ denotes the smallest joint root of the functions

$$
\begin{gathered}
\left(\stackrel{\circ}{f}_{k}^{A}\right)^{r, \alpha, \beta}(\boldsymbol{z}, \boldsymbol{\chi})=\int_{D_{\infty}} \stackrel{\circ}{h}_{f}^{m}\left(\boldsymbol{z}, \boldsymbol{\chi} ; \boldsymbol{w}^{-}, \boldsymbol{w}^{+}, \boldsymbol{x}, s, c, \ell, \tau\right) \mathrm{d} F_{k}^{A}\left(\boldsymbol{w}^{-}, \boldsymbol{w}^{+}, \boldsymbol{x}, s, c, \ell, \tau\right)-z^{r, \alpha, \beta}, \\
\left(\stackrel{\circ}{f}_{k}^{A}\right)^{m}(\boldsymbol{z}, \boldsymbol{\chi})=\int_{D_{\infty}} \stackrel{\circ}{h}_{f}^{m}\left(\boldsymbol{z}, \boldsymbol{\chi} ; \boldsymbol{w}^{-}, \boldsymbol{w}^{+}, \boldsymbol{x}, s, c, \ell, \tau\right) \mathrm{d} F_{k}^{A}\left(\boldsymbol{w}^{-}, \boldsymbol{w}^{+}, \boldsymbol{x}, s, c, \ell, \tau\right)-\chi^{m} .
\end{gathered}
$$

Now choose some $\delta>0$ and $k$ large enough such that $\left(\hat{z}_{k}^{A}, \hat{\chi}_{k}^{A}\right) \geq(1-\delta)(\hat{z}, \hat{\chi})$. Then by (8.8),

$$
\liminf _{k \rightarrow \infty} \stackrel{\circ}{g} k\left(\hat{z}_{k}^{A}, \hat{\chi}_{k}^{A}\right) \geq \liminf _{k \rightarrow \infty} \stackrel{\circ}{g}_{k}^{A}((1-\delta)(\hat{z}, \hat{\chi})) \geq \stackrel{\circ}{g}((1-\delta)(\hat{z}, \hat{\chi}))
$$

and using lower semi-continuity of $\stackrel{\circ}{g}$, as $\delta \rightarrow 0$,

$$
\liminf _{k \rightarrow \infty} \stackrel{\circ}{g}\left(\hat{z}_{k}^{A}, \hat{\chi}_{k}^{A}\right) \geq \stackrel{\circ}{g}(\hat{z}, \hat{\chi}) \text {. }
$$

For the second statement apply a small additional shock to the system in the sense that each solvent institution defaults with probability $\epsilon>0$. Then the analogues of $f^{r, \alpha, \beta}$ and $f^{m}$ in the shocked system are given by

$$
\begin{aligned}
f_{\epsilon}^{r, \alpha, \beta}(z, \chi) & =(1-\epsilon) f^{r, \alpha, \beta}(z, \chi)+\epsilon\left(\mathbb{E}\left[W^{+}\right]-z^{r, \alpha, \beta}\right), \\
f_{\epsilon}^{m}(z, \chi) & =(1-\epsilon) f^{m}(z, \chi)+\epsilon\left(\mathbb{E}\left[X^{m}\right]-\chi^{m}\right) .
\end{aligned}
$$

Denote the analogues of $\left(z^{*}, \chi^{*}\right)$ for these functions by $\left(z^{*}(\epsilon), \chi^{*}(\epsilon)\right)$. Then using (8.9) for $k$ large enough it holds $\left(f_{k}^{B}\right)^{r, \alpha, \beta}\left(z^{*}(\epsilon), \chi^{*}(\epsilon)\right) \leq f^{r, \alpha, \beta}\left(z^{*}(\epsilon), \chi^{*}(\epsilon)\right) / 2<0,(r, \alpha, \beta) \in V$, and $\left(f_{k}^{B}\right)^{m}\left(z^{*}(\epsilon), \chi^{*}(\epsilon)\right) \leq f^{m}\left(z^{*}(\epsilon), \chi^{*}(\epsilon)\right) / 2<0, m \in[M]$. Note that actually it is possible that $f^{r, \alpha, \beta}\left(z^{*}(\epsilon), \chi^{*}(\epsilon)\right)=0$ resp. $f^{m}\left(z^{*}(\epsilon), \chi^{*}(\epsilon)\right)=0$ if $\mathbb{E}\left[W^{+, r, \alpha} \mathbf{1}\{A=\beta\}\right]=0$ resp. $\mathbb{E}\left[X^{m}\right]=0$. In this case, however, the corresponding coordinates $z^{r, \alpha, \beta}$ resp. $\chi^{m}$ are trivial and can be left out. Thus $\left(z^{*}\right)_{k}^{B} \leq z^{*}(\epsilon)$ and $\left(\chi^{*}\right)_{k}^{B} \leq \chi^{*}(\epsilon)$ componentwise and in particular it holds that $\lim \sup _{k \rightarrow \infty}\left(\left(\chi_{k}^{B}\right)^{*}\right)^{m} \leq\left(\chi^{*}\right)^{m}$ for all $m \in[M]$. Using (8.9) again, we now obtain

$$
\limsup _{k \rightarrow \infty} g_{k}^{B}\left(\left(z^{*}\right)_{k}^{B},\left(\chi^{*}\right)_{k}^{B}\right) \leq \limsup _{k \rightarrow \infty} g_{k}^{B}\left(z^{*}(\epsilon), \chi^{*}(\epsilon)\right) \leq g\left(z^{*}(\epsilon), \chi^{*}(\epsilon)\right)
$$

and as $\epsilon \rightarrow 0$ using upper semi-continuity of $g$ we can conclude that

$$
\limsup _{k \rightarrow \infty} g_{k}^{B}\left(\left(z^{*}\right)_{k}^{B},\left(\chi^{*}\right)_{k}^{B}\right) \leq g\left(z^{*}, \chi^{*}\right)
$$

We can then state the proof of the main theorem for general regular vertex sequences:

Proof of Theorem 3.3 For arbitrary $\epsilon>0$ we can apply Lemma 8.3 to derive

$$
\mathbb{P}\left(n^{-1} \mathcal{S}_{n}-\stackrel{\circ}{g}(\hat{z}, \hat{\chi})<-\epsilon\right) \leq \mathbb{P}\left(n^{-1}\left(\mathcal{S}_{k}^{A}\right)_{n}-\stackrel{\circ}{g}(\hat{z}, \hat{\chi})<-\epsilon\right) .
$$

Moreover, it holds $\stackrel{\circ}{g}_{k}^{A}\left(\hat{z}_{k}^{A}, \hat{\chi}_{k}^{A}\right)>\stackrel{\circ}{g}(\hat{z}, \hat{\chi})-\epsilon / 2$ for $k$ large enough by Lemma 8.4 and then

$$
\mathbb{P}\left(n^{-1} \mathcal{S}_{n}-\stackrel{\circ}{g}(\hat{z}, \hat{\chi})<-\epsilon\right) \leq \mathbb{P}\left(n^{-1}\left(\mathcal{S}_{k}^{A}\right)_{n}-\stackrel{\circ}{g}_{k}^{A}\left(\hat{z}_{k}^{A}, \hat{\chi}_{k}^{A}\right)<-\epsilon / 2\right) .
$$

Theorem 8.2 now yields

$$
\mathbb{P}\left(n^{-1} \mathcal{S}_{n}-\stackrel{\circ}{g}(\hat{z}, \hat{\chi})<-\epsilon\right) \rightarrow 0, \quad \text { as } n \rightarrow \infty,
$$


and thus $n^{-1} \mathcal{S}_{n} \geq \stackrel{\circ}{g}(\hat{z}, \hat{\chi})+o_{p}(1)$ as $\epsilon>0$ was arbitrary. Similarly,

$$
\mathbb{P}\left(\chi_{n}^{m}-\hat{\chi}^{m}<-\epsilon\right) \leq \mathbb{P}\left(\left(\chi_{k}^{A}\right)_{n}^{m}-\hat{\chi}^{m}<-\epsilon\right) \leq \mathbb{P}\left(\left(\chi_{k}^{A}\right)_{n}^{m}-\left(\hat{\chi}_{k}^{A}\right)^{m}<-\epsilon / 2\right) \rightarrow 0
$$

as $n \rightarrow \infty$ and hence $\chi_{n}^{m} \geq \hat{\chi}^{m}+o_{p}(1)$ for all $m \in[M]$.

In the same way, by Lemma 8.3

$$
\mathbb{P}\left(n^{-1} \mathcal{S}_{n}-g\left(z^{*}, \chi^{*}\right)>\epsilon\right) \leq \mathbb{P}\left(n^{-1}\left(\mathcal{S}_{k}^{B}\right)_{n}-g\left(z^{*}, \chi^{*}\right)>\epsilon\right)
$$

and hence for $k$ large enough it holds $g_{k}^{B}\left(\left(z^{*}\right)_{k}^{B},\left(\chi^{*}\right)_{k}^{B}\right)<g\left(z^{*}, \chi^{*}\right)+\epsilon / 2$ by Lemma 8.4. Using Theorem 8.2 we thus conclude that

$$
\mathbb{P}\left(n^{-1} \mathcal{S}_{n}-g\left(z^{*}, \chi^{*}\right)>\epsilon\right) \leq \mathbb{P}\left(n^{-1}\left(\mathcal{S}_{k}^{B}\right)_{n}-g_{k}^{B}\left(\left(z^{*}\right)_{k}^{B},\left(\chi^{*}\right)_{k}^{B}\right)>\epsilon / 2\right) \rightarrow 0, \quad \text { as } n \rightarrow \infty,
$$

as well as

$$
\mathbb{P}\left(\chi_{n}^{m}-\left(\chi^{*}\right)^{m}>\epsilon\right) \leq \mathbb{P}\left(\left(\chi_{k}^{B}\right)_{n}^{m}-\left(\chi^{*}\right)^{m}>\epsilon\right) \leq \mathbb{P}\left(\left(\chi_{k}^{A}\right)_{n}^{m}-\left(\left(\chi^{*}\right)_{k}^{A}\right)^{m}>\epsilon / 2\right) \rightarrow 0 .
$$

\subsection{Proofs for Sect. 4}

We keep the notation $g, f^{r, \alpha, \beta}, f^{m}, z^{*}, \chi^{*}, \stackrel{\circ}{g}, \stackrel{\circ}{f} r, \alpha, \beta, \stackrel{\circ}{f}, \hat{z}$ and $\hat{\chi}$ for the quantities from Sect. 3 for the unshocked system and add the index $\cdot_{L}$ to indicate the corresponding quantities and functions in the system shocked by $L$.

Proof of Theorem 4.2 For arbitrary $\alpha>0$, we derive

$$
\begin{aligned}
& f_{L}^{r, \alpha, \beta}(z, \chi) \\
& =\mathbb{E}\left[W^{+, r, \alpha} \mathbb{P}\left(\sum_{s \in[R]} s \operatorname{Poi}\left(\sum_{\gamma \in[T]} W^{-, s, \gamma} z^{s, \beta, \gamma}\right) \geq C-L-X \cdot h(\boldsymbol{\chi})\right) \mathbf{1}\{A=\beta\}\right]-z^{r, \alpha, \beta} \\
& \leq \mathbb{E}\left[W^{+, r, \alpha} \mathbf{1}\{L \geq \alpha C\}\right]-z^{r, \alpha, \beta} \\
& \quad+\mathbb{E}\left[W^{+, r, \alpha} \mathbb{P}\left(\sum_{s \in[R]} s \operatorname{Poi}\left(\sum_{\gamma \in[T]} W^{-, s, \gamma} z^{s, \beta, \gamma}\right) \geq C(1-\alpha)-X \cdot h(\chi)\right) \mathbf{1}\{A=\beta\}\right] .
\end{aligned}
$$

Using that $\mathbb{E}[L / C]<\delta$, we derive with Markov's inequality that $\mathbb{P}(L \geq \alpha C)<\delta / \alpha$ and as $\mathbb{E}\left[W^{+, r, \alpha}\right]<\infty$ it thus holds that $\mathbb{E}\left[W^{+, r, \alpha} \mathbf{1}\{L \geq \alpha C\}\right] \leq \gamma / 3$ for any arbitrary $\gamma>0$ if we choose $\delta>0$ small enough. Also the second summand in above inequality can be bounded by $f^{r, \alpha, \beta}(z, \chi)+\gamma / 3$ if $\alpha$ is chosen small enough using the dominated convergence theorem.

Let now $((\tilde{\boldsymbol{z}}(\gamma), \tilde{\boldsymbol{\chi}}(\gamma)))_{\gamma>0} \subset \mathbb{R}_{+, 0}^{V} \times \mathbb{R}_{+, 0}^{M}$ be such that $f^{r, \alpha, \beta}(\tilde{\boldsymbol{z}}(\gamma), \tilde{\boldsymbol{\chi}}(\gamma))=-\gamma$ for all $(r, \alpha, \beta) \in V$ resp. $f^{m}(\tilde{z}(\gamma), \tilde{\chi}(\gamma))=-\gamma$ for all $m \in[M]$, which exists analogue to [18, Remark EC. 1] (extending it by the $z$-coordinates). By above result then $f_{L}^{r, \alpha, \beta}(\tilde{z}(\gamma), \tilde{\chi}(\gamma)) \leq$ $-\gamma / 3<0$ for $\delta$ small enough. Similarly, one derives that $f_{L}^{m}(\tilde{z}(\gamma), \tilde{\chi}(\gamma)) \leq-\gamma / 3<0$ for $\delta$ small enough. We can thus conclude that $\left(z_{L}^{*}, \chi_{L}^{*}\right)<(\tilde{z}(\gamma), \tilde{\chi}(\gamma))$. However, by [18, Remark EC. 1] we further know that $(\tilde{z}(\gamma), \tilde{\chi}(\gamma)) \rightarrow\left(z^{*}, \chi^{*}\right)$ and hence by upper semicontinuity of $g$ and possibly further decreasing $\delta$ it holds

$$
g\left(z_{L}^{*}, \chi_{L}^{*}\right) \leq g\left(z^{*}, \chi^{*}\right)+\epsilon / 3=\epsilon / 3 .
$$

By similar means as for $f^{r, \alpha, \beta}$ and $f^{m}$ above, we also derive that $g_{L}(z, \chi) \leq \epsilon / 3+g(z, \chi)$ for $\delta$ small enough. By Theorem 3.3 we can thus conclude that w.h.p.

$$
n^{-1} \mathcal{S}_{n, L} \leq g_{L}\left(z_{L}^{*}, \chi_{L}^{*}\right)+\epsilon / 3 \leq g\left(z_{L}^{*}, \chi_{L}^{*}\right)+2 \epsilon / 3 \leq \epsilon .
$$


Now let $\delta$ small enough such that also $\left(\chi_{L}^{*}\right)^{m} \leq\left(\chi^{*}\right)^{m}+\epsilon / 2$. Applying Theorem 3.3 we thus derive that w.h.p.

$$
\chi_{n, L}^{m} \leq\left(\chi_{L}^{*}\right)^{m}+\epsilon / 2 \leq\left(\chi^{*}\right)^{m}+\epsilon .
$$

Proof of Theorem 4.6 Define for $\epsilon>0$ and $I_{V} \subset V$ resp. $I_{M} \subset[M]$ the set

$$
\begin{aligned}
T(\epsilon, I):= & \bigcap_{(r, \alpha, \beta) \in I_{V}}\left\{(z, \chi) \in \mathbb{R}_{+, 0}^{V} \times \mathbb{R}_{+, 0}^{M}: \stackrel{\circ}{f}^{r, \alpha, \beta}(z, \chi) \leq-\epsilon\right\} \\
& \cap \bigcap_{(s, \theta, \lambda) \in I_{V}^{c}}\left\{(z, \chi) \in \mathbb{R}_{+, 0}^{V} \times \mathbb{R}_{+, 0}^{M}: z^{s, \theta, \lambda} \geq \mathbb{E}\left[W^{+, s, \theta} \mathbf{1}\{A=\lambda\}\right]\right\} \\
& \cap \bigcap_{m \in I_{M}}\left\{(z, \chi) \in \mathbb{R}_{+, 0}^{V} \times \mathbb{R}_{+, 0}^{M}: f^{m}(z, \chi) \leq-\epsilon\right\} \\
& \cap \bigcap_{k \in I_{M}^{c}}\left\{(z, \chi) \in \mathbb{R}_{+, 0}^{V} \times \mathbb{R}_{+, 0}^{M}: \chi^{k} \geq \mathbb{E}\left[X^{k}\right]\right\}
\end{aligned}
$$

where we denote $I_{V}^{c}:=V \backslash I_{V}$ and $I_{M}^{c}=[M] \backslash I_{M}$. Moreover, denote by $(\hat{z}(\epsilon, I), \hat{\chi}(\epsilon, I)) \epsilon$ $\mathbb{R}_{+, 0}^{V} \times \mathbb{R}_{+, 0}^{M}$ the smallest vector such that $\stackrel{\circ}{f}^{r, \alpha, \beta}(\hat{z}(\epsilon, I), \hat{\chi}(\epsilon, I))=-\epsilon$ for $(r, \alpha, \beta) \in I_{V}$ and $\hat{z}^{s, \theta, \lambda}(\epsilon, I)=\mathbb{E}\left[W^{+, r, \alpha} \mathbf{1}\{A=\beta\}\right]$ for $(s, \theta, \lambda) \in I_{V}^{c}$ resp. $f^{m}(\hat{z}(\epsilon, I), \hat{\chi}(\epsilon, I))=-\epsilon$ for $m \in I_{M}$ and $\hat{\chi}^{k}(\epsilon, I)=\mathbb{E}\left[X^{k}\right]$ for $k \in I_{M}^{c}$. The existence of such a vector is ensured analogue to Lemma 3.2. In particular, it then holds $(\hat{z}(\epsilon, I), \hat{\chi}(\epsilon, I)) \in T(\epsilon, I)$ and by the construction of $(\hat{z}(\epsilon, I), \hat{\chi}(\epsilon, I))$ analogue to Lemma 3.2 we obtain that $(\hat{z}(\epsilon, I), \hat{\chi}(\epsilon, I)) \leq$ $(z, \chi)$ for any other $(z, \chi) \in T(\epsilon, I)$

In particular, this implies that $(\hat{z}(\epsilon, I), \hat{\chi}(\epsilon, I))$ is non-decreasing in $\epsilon$ and thus continuous for almost every $\epsilon>0$. As moreover, the expressions $f^{r, \alpha, \beta}(\hat{z}(\epsilon, I), \hat{\chi}(\epsilon, I))+\hat{z}^{r, \alpha, \beta}(\epsilon, I)$ and $f^{m}(\hat{z}(\epsilon, I), \hat{\chi}(\epsilon, I))+\hat{\chi}^{m}(\epsilon, I)$ are bounded and increasing in $\epsilon$, we derive that for almost every $\epsilon>0$ and $\delta>0$, we can choose $\gamma>0$ small enough such that

$$
\begin{aligned}
& f^{r, \alpha, \beta}(\hat{z}(\epsilon, I), \hat{\chi}(\epsilon, I))+\hat{z}^{r, \alpha, \beta}(\epsilon, I) \\
& \quad \leq f^{r, \alpha, \beta}(\hat{z}(\epsilon-\gamma, I), \hat{\chi}(\epsilon-\gamma, I))+\hat{z}^{r, \alpha, \beta}(\epsilon-\gamma, I)+\delta
\end{aligned}
$$

and

$$
f^{m}(\hat{z}(\epsilon, I), \hat{\chi}(\epsilon, I))+\hat{\chi}^{m}(\epsilon, I) \leq f^{m}(\hat{z}(\epsilon-\gamma, I), \hat{\chi}(\epsilon-\gamma, I))+\hat{\chi}^{m}(\epsilon-\gamma, I)+\delta .
$$

If $(r, \alpha, \beta) \in I_{V}$, as $\hat{z}(\epsilon, I)$ is strictly increasing, we derive that

$$
\begin{aligned}
f^{r, \alpha, \beta}(\hat{z}(\epsilon, I), \hat{\chi}(\epsilon, I)) & \leq f^{r, \alpha, \beta}(\hat{z}(\epsilon, I), \hat{\chi}(\epsilon, I)) \\
& \leq f^{r, \alpha, \beta}(\hat{z}(\epsilon-\gamma, I), \hat{\chi}(\epsilon-\gamma, I))+\delta \\
& \leq f^{r, \alpha, \beta}(\hat{z}(\epsilon-\gamma, I), \hat{\chi}(\epsilon, I))+\delta \\
& \leq f^{r, \alpha, \beta}(\hat{z}(\epsilon, I), \hat{\chi}(\epsilon, I))+\delta
\end{aligned}
$$

and choosing $\delta$ arbitrarily small we conclude that for almost every $\epsilon>0$,

$$
f^{r, \alpha, \beta}(\hat{z}(\epsilon, I), \hat{\chi}(\epsilon, I))=\stackrel{\circ}{f}^{r, \alpha, \beta}(\hat{z}(\epsilon, I), \hat{\chi}(\epsilon, I))=-\epsilon .
$$

Moreover, if $m \in I_{M}$, then $\hat{\chi}^{m}(\epsilon, I)$ is strictly increasing and by the assumption of $h^{m}(\chi)$ being strictly increasing in $\chi^{m}$, we derive for $x^{m}>0$ that

$$
\rho_{\alpha}\left(\frac{\ell+\boldsymbol{x} \cdot h(\hat{\chi}(\epsilon-\gamma, I))}{c}\right) \leq \stackrel{\circ}{\rho}_{\alpha}\left(\frac{\ell+\boldsymbol{x} \cdot h(\hat{\chi}(\epsilon, I))}{c}\right)
$$


entry-wise for all $\alpha \in[T]$. Hence similarly as above, $f^{m}(\hat{z}(\epsilon, I), \hat{\chi}(\epsilon, I))=\stackrel{\circ}{f}^{m}(\hat{z}(\epsilon, I)$, $\hat{\chi}(\epsilon, I))=-\epsilon$.

Let us now show that $\left(z^{*}, \chi^{*}\right) \leq(\hat{z}(\epsilon, I), \hat{\chi}(\epsilon, I))$. To this end, suppose that we could choose some $(z, \chi) \in P_{0}$ such that $z^{r, \alpha, \beta}>\hat{z}^{r, \alpha, \beta}(\epsilon, I)$ for some $(r, \alpha, \beta) \in V$ or $\chi^{m}>$ $\hat{\chi}(\epsilon, I)$ for some $m \in[M]$. Then by $P_{0} \subset[0, \zeta] \times[\mathbf{0}, \eta]$, where $\zeta^{r, \alpha, \beta}=\mathbb{E}\left[W^{+, r, \alpha} \mathbf{1}\{A=\right.$ $\beta\}]$ and $\eta^{m}=\mathbb{E}\left[X^{m}\right]$, and monotonicity of the functions $f^{r, \alpha, \beta}$ resp. $f^{m}$ we would derive the existence of a point $P_{0} \ni(\tilde{z}, \tilde{\chi}) \leq(\hat{z}(\epsilon, I), \hat{\chi}(\epsilon, I))$ such that either $\tilde{z}^{r, \alpha, \beta}=\hat{z}^{r, \alpha, \beta}(\epsilon, I)$ for some $(r, \alpha, \beta) \in I_{V}$ or $\tilde{\chi}^{m}=\hat{\chi}^{M}(\epsilon, I)$ for some $m \in I_{M}$. But then $f^{r, \alpha, \beta}(\tilde{z}, \tilde{\chi}) \leq$ $f^{r, \alpha, \beta}(\hat{z}(\epsilon, I), \hat{\chi}(\epsilon, I))=-\epsilon \operatorname{resp} . f^{m}(\tilde{z}, \tilde{\chi}) \leq f^{m}(\hat{z}(\epsilon, I), \hat{\chi}(\epsilon, I))=-\epsilon$ and thus a contradiction to $(\tilde{z}, \tilde{\chi}) \in P_{0}$. It must therefore hold $\left(z^{*}, \chi^{*}\right) \leq(\hat{z}(\epsilon, I), \hat{\chi}(\epsilon, I))$. Let now for given shock $L$,

$I_{M}:=\left\{m \in[M]: \hat{\chi}_{L}^{m}<\mathbb{E}\left[X^{m}\right]\right\} \quad$ and $I_{V}:=\left\{(r, \alpha, \beta) \in V: \hat{z}_{L}^{r, \alpha, \beta}<\mathbb{E}\left[W^{+, r, \alpha} \mathbf{1}\{A=\beta\}\right]\right.$.

If $I_{V}=\varnothing$ and $I_{M}=\emptyset$ the result is trivial, so assume that either $I_{V} \neq \varnothing$ or $I_{M} \neq \varnothing$. For $m \in I_{M}$, using $f_{L}^{m}\left(\hat{z}_{L}, \hat{\chi}_{L}\right)=0$ we then derive by independence of $L / C$ and $\left(\boldsymbol{W}^{-}, \boldsymbol{W}^{+}, \boldsymbol{X}, S, C, A\right)$

$$
\stackrel{\circ}{f}^{m}\left(\hat{z}_{L}, \hat{\chi}_{L}\right)=\frac{\stackrel{\circ}{f}_{L}^{m}\left(\hat{z}_{L}, \hat{\chi}_{L}\right)-\mathbb{P}(L=2 C)\left(\mathbb{E}\left[X^{m}\right]-\hat{\chi}_{L}^{m}\right)}{\mathbb{P}(L=0)}<0
$$

and analogously $\stackrel{\circ}{f}^{r, \alpha, \beta}\left(\hat{z}_{L}, \hat{\chi}_{L}\right)<0$ for $(r, \alpha, \beta) \in I_{V}$. For the choice

$$
\epsilon:=-\max \left\{\max _{(r, \alpha, \beta) \in I_{V}} f^{r, \alpha, \beta}\left(\hat{z}_{L}, \hat{\chi}_{L}\right), \max _{m \in I_{M}} \stackrel{\circ}{m}^{m}\left(\hat{z}_{L}, \hat{\chi}_{L}\right)\right\}>0
$$

it then holds $\left(\hat{z}_{L}, \hat{\chi}_{L}\right) \in T(\epsilon, I)$ and further $\left(\hat{z}_{L}, \hat{\chi}_{L}\right) \geq(\hat{z}(\epsilon, I), \hat{\chi}(\epsilon, I)) \geq\left(z^{*}, \chi^{*}\right)$. We can then apply Theorem 3.3 and conclude

$$
n^{-1} \mathcal{S}_{n, L} \geq \stackrel{\circ}{g}_{L}\left(\hat{z}_{L}, \hat{\chi}_{L}\right)+o_{p}(1) \geq \stackrel{\circ}{g}\left(z^{*}, \chi^{*}\right)+o_{p}(1)
$$

as well as

$$
\chi_{n, L}^{m} \geq \hat{\chi}_{L}^{m}+o_{p}(1) \geq\left(\chi^{*}\right)^{m}+o_{p}(1) .
$$

\section{Glossary}

$o(1)$

$o_{p}(1)$

$\mathbb{R}_{+, 0}, \mathbb{R}_{+, \infty}$

$[\boldsymbol{a}, \boldsymbol{b}]$

$n \in \mathbb{N}$

$M \in \mathbb{N}$

$[n]=\{1, \ldots, n\}$

$x_{i}^{m} \in \mathbb{R}_{+, 0}$

$\boldsymbol{x}_{i}=\left(x_{i}^{1}, \ldots, x_{i}^{M}\right) \in \mathbb{R}_{+, 0}^{M}$

$c_{i} \in \mathbb{R}_{+, \infty}$

$\ell_{i} \in \mathbb{R}_{+, 0}$ the Landau notation for a term that converges to 0 (e.g. as $n \rightarrow \infty)$

the Landau notation for a term that converges to 0 in probability (e.g. as $n \rightarrow \infty$ )

positive real numbers with 0 resp. $\infty$

cuboid spanned by vectors $\boldsymbol{a}, \boldsymbol{b} \in \mathbb{R}^{d}$ consisting of all $x \in \mathbb{R}^{d}$ such that $a_{i} \leq x_{i} \leq b_{i}, i=1, \ldots, d$

number of banks in the system

number of assets/asset classes

set of banks

number of shares of asset $m \in[M]$ held by bank $i \in[n]$ vector of asset holdings of bank $i \in[n]$

equity of bank $i \in[n]$

exogenous losses of bank $i \in[n]$ 
$w_{i}^{-, \alpha}, w_{i}^{+, \alpha} \in \mathbb{R}_{+, 0} \in \mathbb{R}_{+, 0}$

$\boldsymbol{w}_{i}^{-}=\left(w_{i}^{-, 1}, \ldots, w_{i}^{-, T}\right) \in \mathbb{R}_{+, 0}^{T}$

$\boldsymbol{w}_{i}^{+}=\left(w_{i}^{+, 1}, \ldots, w_{i}^{+, T}\right) \in \mathbb{R}_{+, 0}^{T}$

$X_{i, j}^{r}$

$\left(\boldsymbol{W}_{n}^{-}, \boldsymbol{W}_{n}^{+}, \boldsymbol{X}_{n}, S_{n}, C_{n}, A_{n}\right)$

$\left(\boldsymbol{W}^{-}, \boldsymbol{W}^{+}, \boldsymbol{X}, S, C, A\right)$

$\rho_{\beta}: \mathbb{R}_{+, 0} \rightarrow[0,1]^{M}$

$\stackrel{\circ}{\rho} \beta_{\beta}(u)=\lim _{\epsilon \rightarrow 0+} \rho_{\beta}((1-\epsilon) u)$

$h^{m}: \mathbb{R}_{+, 0}^{M} \rightarrow[0,1]$

$h(y)=\left(h^{1}(y), \ldots, h^{M}(y)\right)$

$\chi_{n} \in \mathbb{R}_{+, 0}^{M}$

$\mathcal{D}_{n} \subset[n]$

$Q_{s} \sim \operatorname{Poi}\left(q_{s}\right), s \in[R]$ in- resp. out-weight of bank $i \in[n]$ towards bank of type $\alpha \in[T]$

vector of in-weights of bank $i \in[n]$ towards banks of types $\alpha=1, \ldots, T$

vector of out-weights of bank $i \in[n]$ towards banks of types $\alpha=1, \ldots, T$

indicator random variable for an edge of size $r \in[R]$ going from bank $i \in[n]$ to bank $j \in[n]$

random vector distributed according to the empirical distribution of $\left\{\left(\boldsymbol{w}_{i}^{-}, \boldsymbol{w}_{i}^{+}, \boldsymbol{x}_{i}, s_{i}, c_{i}, \ell_{i}\right)\right\}_{i \in[n]}$

limit in distribution of $\left(\boldsymbol{W}_{n}^{-}, \boldsymbol{W}_{n}^{+}, \boldsymbol{X}_{n}, S_{n}, C_{n}, A_{n}\right)$

non-decreasing, right-continuous sale functions for each type $\beta \in[T]$

left-continuous modification of $\rho_{\beta}, \beta \in[T]$

non-decreasing (componentwise) relative loss function of asset $m \in[M]$

vector of loss functions

vector of finally sold shares divided by $n$

set of finally defaulted banks $Y_{r, \beta}:=\sum_{\gamma \in[T]} W^{-, r, \gamma}$ $z^{r, \beta, \gamma}, \quad r \in[R], \beta \in[T]$,

Independent Poisson for $\left\{q_{s}\right\}_{s \in[R]} \subset \mathbb{R}_{+, 0}$

$$
\begin{aligned}
& \psi\left(q_{1}, \ldots, q_{R} ; t\right):=\mathbb{P}\left(\sum_{s \in[R]} s Q_{s} \geq t\right) \\
& \phi_{\alpha}\left(q_{1}, \ldots, q_{R} ; \ell, c\right):=\mathbb{E}\left[\rho_{\alpha}\left(\frac{\sum_{s \in[R]} s Q_{s}+\ell}{c}\right)\right], \quad \alpha \in[T] . \\
& \psi\left(z, \chi ; w^{-}, \boldsymbol{x}, c, \ell\right)=\mathbb{P}\left(\operatorname{Poi}\left(w^{-} z\right) \geq c-\ell-\boldsymbol{x} \cdot h(\chi)\right) \\
& \phi_{\beta}\left(z, \chi ; w^{-}, \boldsymbol{x}, c, \ell\right)=\mathbb{E}\left[\rho_{\beta}\left(\frac{\operatorname{Poi}\left(w^{-} z\right)+\ell+\boldsymbol{x} \cdot h(\chi)}{c}\right)\right], \quad \beta \in[T] \\
& g(z, \chi):=\sum_{\beta \in[T]} \mathbb{E}\left[S \psi\left(Y_{1, \beta}, \ldots, Y_{R, \beta} ; C-L-\boldsymbol{X} \cdot h(\chi)\right) \mathbf{1}\{A=\beta\}\right], \\
& f^{r, \alpha, \beta}(z, \chi):=\mathbb{E}\left[W^{+, r, \alpha} \psi\left(Y_{1, \beta}, \ldots, Y_{R, \beta} ; C-L-\boldsymbol{X} \cdot h(\chi)\right) \mathbf{1}\{A=\beta\}\right] \\
& \quad-z^{r, \alpha, \beta},(r, \alpha, \beta) \in V \\
& f^{m}(z, \chi):=\sum_{\beta \in[T]} \mathbb{E}\left[X^{m} \phi_{\beta}^{m}\left(Y_{1, \beta}, \ldots, Y_{R, \beta} ; L+\boldsymbol{X} \cdot h(\chi), C\right) \mathbf{1}\{A=\beta\}\right]-\chi^{m}, m \in[M] \\
& P:=\bigcap_{(r, \alpha, \beta) \in V}\left\{(z, \chi) \in \mathbb{R}_{+, 0}^{V} \times \mathbb{R}_{+, 0}^{M}: f^{r, \alpha, \beta}(z, \chi) \geq 0\right\} \\
& \cap \bigcap_{m \in[M]}\left\{(z, \chi) \in \mathbb{R}_{+, 0}^{V} \times \mathbb{R}_{+, 0}^{M}: f^{m}(z, \chi) \geq 0\right\}
\end{aligned}
$$$$
P_{0}
$$$$
\text { connected component of } P \text { containing } \mathbf{0}
$$ 


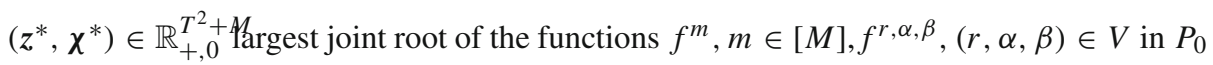

$$
\begin{aligned}
& \stackrel{\circ}{\phi}_{\alpha}\left(q_{1}, \ldots, q_{R} ; \ell, c\right):=\mathbb{E}\left[\stackrel{\circ}{\rho}_{\alpha}\left(\frac{\sum_{s \in[R]} s Q_{s}+\ell}{c}\right)\right], \quad \alpha \in[T] \\
& \stackrel{\circ}{g}(z, \chi):=\sum_{\beta \in[T]} \mathbb{E}\left[S \psi\left(Y_{1, \beta}, \ldots, Y_{R, \beta} ; C-L-\boldsymbol{X} \cdot h(\chi)\right) \mathbf{1}\{A=\beta\}\right], \\
& \stackrel{\circ}{f}^{r, \alpha, \beta}(z, \chi):=\mathbb{E}\left[W^{+, r, \alpha} \psi\left(Y_{1, \beta}, \ldots, Y_{R, \beta} ; C-L-\boldsymbol{X} \cdot h(\chi)\right) \mathbf{1}\{A=\beta\}\right] \\
& \quad-z^{r, \alpha, \beta},(r, \alpha, \beta) \in V, \\
& \stackrel{\circ}{f}(z, \chi):=\sum_{\beta \in[T]} \mathbb{E}\left[X^{m} \stackrel{\circ}{\phi}_{\beta}^{m}\left(Y_{1, \beta}, \ldots, Y_{R, \beta} ; L+\boldsymbol{X} \cdot h(\chi), C\right) \mathbf{1}\{A=\beta\}\right]-\chi^{m}, m \in[M] \\
& \stackrel{\circ}{P}:=\bigcap_{(r, \alpha, \beta) \in V}\left\{(z, \chi) \in \mathbb{R}_{+, 0}^{V} \times \mathbb{R}_{+, 0}^{M}: f^{r, \alpha, \beta}(z, \chi) \geq 0\right\} \\
& \cap \bigcap_{m \in[M]}\left\{(z, \chi) \in \mathbb{R}_{+, 0}^{V} \times \mathbb{R}_{+, 0}^{M}: f^{m}(z, \chi) \geq 0\right\}
\end{aligned}
$$

$\stackrel{\circ}{P}_{0} \quad$ connected component of $\stackrel{\circ}{P}$ containing $\mathbf{0}$

$(\hat{z}, \hat{\chi}) \in \mathbb{R}_{+, 0}^{T^{2}+M}$ smallest joint root of the functions $f^{m}, m \in[M]_{0}$

\section{References}

1. Acemoglu, D., Ozdaglar, A., Tahbaz-Salehi, A.: Systemic risk and stability in financial networks. Am. Econ. Rev. 105(2), 564-608 (2015)

2. Allen, F., Gale, D.: Financial contagion. J. Polit. Econ. 108(1), 1-33 (2000)

3. Amini, H., Cont, R., Minca, A.: Resilience to contagion in financial networks. Math. Finance 26(2), 329-365 (2016)

4. Armenti, Y., Crpey, S., Drapeau, S., Papapantoleon, A.: Multivariate shortfall risk allocation and systemic risk. SIAM J. Financ. Math. 9, 90-126 (2018)

5. Bernard, B., Capponi, A., Stiglitz, J.E.: Bail-ins and bail-outs: incentives, connectivity, and systemic stability. Working Paper 23747, National Bureau of Economic Research, August (2017)

6. Biagini, F., Fouque, J.-P., Frittelli, M., Meyer-Brandis, T.: A unified approach to systemic risk measures via acceptance sets. Math. Finance 29(1), 329-367 (2019)

7. Blondel, V.D., Guillaume, J.-L., Lambiotte, R., Lefebvre, E.: Fast unfolding of communities in large networks. J. Stat. Mech. Theory Exp. 2008(10), P10008 (2008)

8. Caccioli, F., Shrestha, M., Moore, C., Farmer, J.D.: Stability analysis of financial contagion due to overlapping portfolios. J. Bank. Finance 46, 233-245 (2014)

9. Capponi, A., Chen, P.-C., Yao, D.D.: Liability concentration and systemic losses in financial networks. Oper. Res. 64(5), 1121-1134 (2016)

10. Capponi, A., Larsson, M.: Price contagion through balance sheet linkages. Rev. Asset Pricing Stud. 5(2), 227-253 (2015)

11. Chen, C., Iyengar, G., Moallemi, C.: An axiomatic approach to systemic risk. Manag. Sci. 59(6), 13731388 (2013)

12. Cifuentes, R., Ferrucci, G., Shin, H.S.: Liquidity risk and contagion. J. Eur. Econ. Assoc. 3(2-3), 556-566 (2005)

13. Clauset, A., Newman, M.E.J., Moore, C.: Finding community structure in very large networks. Phys. Rev. E 70, 066111 (2004)

14. Cont, R., Moussa, A., Santos, E.: Network structure and systemic risk in banking systems. In: Fouque, J.-P., Langsam, J. (eds.) Handbook on Systemic Risk. Cambridge University Press, Cambridge (2013) 
15. Copic, J., Jackson, M.O., Kirman, A.: Identifying community structures from network data via maximum likelihood methods. BE J. Theor. Econ. 9(1), 6 (2009)

16. Detering, N., Meyer-Brandis, T., Panagiotou, K.: Bootstrap percolation in directed and inhomogeneous random graphs. Electron. J. Comb. 26(3), 1-43 (2019)

17. Detering, N., Meyer-Brandis, T., Panagiotou, K., Ritter, D.: Financial contagion in a generalized stochastic block model (2018). arXiv:1803.08169

18. Detering, N., Meyer-Brandis, T., Panagiotou, K., Ritter, D.: Suffocating fire sales. Preprint (2018)

19. Detering, N., Meyer-Brandis, T., Panagiotou, K., Ritter, D.: Managing default contagion in inhomogeneous financial networks. SIAM J. Financ. Math. 10(2), 578-614 (2019)

20. Eisenberg, L., Noe, T.H.: Systemic risk in financial systems. Manag. Sci. 47(2), 236-249 (2001)

21. Feinstein, Z., Rudloff, B., Weber, S.: Measures of systemic risk. SIAM J. Financ. Math. 8(1), 672-708 (2017)

22. Fortunato, S., Hric, D.: Community detection in networks: a user guide. Phys. Rep. 659, 1-44 (2016)

23. Gai, P., Kapadia, S.: Contagion in financial networks. Proc. R. Soc. A 466, 2401-2423 (2010)

24. Glasserman, P., Young, H.P.: How likely is contagion in financial networks? J. Bank. Finance 50, 383-399 (2015)

25. Greenwood, R., Landier, A., Thesmar, D.: Vulnerable banks. J. Financ. Econ. 115(3), 471-485 (2015)

26. Hoffmann, H., Meyer-Brandis, T., Svindland, G.: Risk-consistent conditional systemic risk measures. Stoch. Process. Appl. 126(7), 2014-2037 (2016)

27. Holme, P.: Core-periphery organization of complex networks. Phys. Rev. E 72, 046111 (2005)

28. Hurd, T.R.: Contagion! Systemic Risk in Financial Networks. Springer, Berlin (2016)

29. Kromer, E., Overbeck, L., Zilch, K.: Systemic risk measures on general measurable spaces. Math. Meth. Operations Res. 84(2), 323-357 (2016)

30. Online (2017). www.creditfixings.com/information/affiliations/fixings/auctions/2008/lehbro-res.shtml. Accessed April 2017

31. Ritter, D.: Mathematical modeling of systemic risk in financial networks: managing default contagion and fire sales. Ph.D. Thesis, University of Munich (2019). arXiv:1911.07313

32. Rogers, L.C.G., Veraart, L.A.M.: Failure and rescue in an interbank network. Manag. Sci. 59(4), 882-898 (2013)

33. Rombach, P., Porter, M., Fowler, J., Mucha, P.: Core-periphery structure in networks (revisited). SIAM Rev. 59(3), 619-646 (2017)

34. Weber, S., Weske, K.: The joint impact of bankruptcy costs, fire sales and cross-holdings on systemic risk in financial networks. Prob. Uncert. Quant. Risk 2, 9 (2017)

35. Wormald, N.C.: Differential equations for random processes and random graphs. Ann. Appl. Prob. 5(4), $1217-1235$ (1995)

36. Zhang, P., Moore, C.: Scalable detection of statistically significant communities and hierarchies, using message passing for modularity. Proc. Natl. Acad. Sci. 111(51), 18144-18149 (2014)

37. Zhao, Y., Levina, E., Zhu, J.: Community extraction for social networks. Proc. Natl. Acad. Sci. 108(18), 7321-7326 (2011)

Publisher's Note Springer Nature remains neutral with regard to jurisdictional claims in published maps and institutional affiliations. 